\title{
Kapitel 4: Die Wiederherstellung der Nutzungsmöglichkeit des digitalen Spiels
}

Nachdem der Support der Online-Komponenten beendet wurde, entfällt die Nutzungsmöglichkeit des digitalen Spiels gänzlich oder zum Teil. ${ }^{778}$ Möglicherweise möchte der Erwerber das digitale Spiel aber sowohl im Einzel- als auch im Mehrspielermodus weiternutzen. So ist in der Einleitung dieser Arbeit deutlich geworden, dass private Spieler, Forscher und Gedächtnisinstitutionen durchaus ein Interesse an einer längerfristigen, über den Zeitraum des offiziellen Supports hinausgehenden Nutzungsmöglichkeit des digitalen Spiels haben können.

Es ist bereits in Kapitel 1 dargestellt worden, welche Maßnahmen private Spieler unternehmen müssen, um die Nutzungsmöglichkeit supportloser digitaler Spiele wiederherzustellen. ${ }^{779}$ Sie umfasst einerseits die Eliminierung von Online-DRM-Systemen und andererseits die Wiederherstellung des Online-Mehrspielermodus. Über die Rechtmäßigkeit dieser Maßnahmen herrscht bei den genannten Akteuren große Unsicherheit.

Es stellt sich zunächst die Frage, ob der Erwerber nach dem Ende des Supports überhaupt noch zur Nutzung des digitalen Spiels berechtigt ist. Denn die Wiedernutzbarmachung hat nur wenig Sinn, wenn der Spieler nach dem Ende des Supports der Online-Komponenten ohnehin nicht mehr zur Nutzung des Spiels berechtigt ist (A.).

Daraufhin werden die Eliminierung der Online-DRM-Systeme sowie die dafür erforderlichen Maßnahmen auf ihre Rechtmäßigkeit hin untersucht (B.).

Im Anschluss daran wird die Wiederherstellung des Online-Mehrspielermodus rechtlich untersucht (C.).

Die Frage, ob Erwerber die Nutzungsmöglichkeit supportloser digitaler Spiele eigenhändig wiederherstellen dürfen, war ferner in den USA Gegenstand des sog. Sixth Triennial Section 1201 Proceeding, welches von 2014 bis 2015 vor dem US Copyright Office stattfand. Der Verlauf dieses Verfahrens sowie dessen Ergebnisse sollen am Schluss dieses Kapitels (D.) dargestellt werden.

\footnotetext{
778 Siehe dazu die Einleitung.

779 Siehe dazu ausführlich oben, S. 23 ff.
} 


\section{A. Die urheberrechtliche Nutzungsberechtigung des Erwerbers}

\section{Der Schutz digitaler Spiele nach dem UrhG}

Digitale Spiele stellen komplexe multimediale Objekte dar, die als Ganzes oder in Teilen Urheberrechtsschutz genießen können. Sie setzen sich aus einer Vielzahl von unterschiedlichen Bestandteilen zusammen. Essenziell ist dabei die dem Spiel zugrunde liegende Software, welche den Spielablauf steuert und Voraussetzung für das Funktionieren des Spiels ist. ${ }^{780}$ Darüber hinaus wird auf einem Bildschirm der Spielverlauf in Form von bewegten Bildern wiedergegeben. Schließlich können auch noch weitere Bestandteile wie Musik oder Text hinzukommen, aus denen sich das digitale Spiel zusammensetzt. ${ }^{781}$

Um als Werk nach dem UrhG Schutz zu genießen, muss das digitale Spiel eine persönliche geistige Schöpfung gemäß $§ 2$ Abs. 2 UrhG darstellen. Dies dürfte bei modernen digitalen Spielen regelmäßig der Fall sein. ${ }^{782}$ Doch auch wenn die Werkqualität im Einzelfall zu verneinen sein sollte, können digitale Spiele immer noch als Laufbilder gem. § 95 UrhG geschützt sein. ${ }^{783}$

Für den Fall, dass das digitale Spiel eine persönliche geistige Schöpfung darstellt, besteht in Rechtsprechung und Literatur weitgehend Einigkeit darüber, dass ihm als Ganzes Schutz als Filmwerk gemäß § 2 Abs. 1 Nr. 6 UrhG, oder zumindest als Werk, welches ähnlich wie ein Filmwerk geschaffen wird, zukommt. ${ }^{784}$ Die Software, die für die Steuerung des Spielablaufs verantwortlich ist, kann als „Computerprogramm“ gemäß den $\S \S 69$ a ff. UrhG geschützt sein. ${ }^{785}$ Unter Urheberrechtsschutz können darüber

780 Vgl. Rauda, Computerspiele, Rn. 70; Förster, in Duisberg/Picot, Recht der Computer- und Videospiele, Kap. 2, Rn. 1.

781 Rauda, Computerspiele, Rn. 70.

782 Fromm/Nordemann/Nordemann, § 2 UrhG, Rn. 204; Bullinger/Czychowski, GRUR 2011, 19 , 23; OLG Hamburg, GRUR 1983, 436, 437; GRUR 1990, 127, 128 - Super Mario III; zumindest in Bezug auf das dem Spiel zugrunde liegende Computerprogramm Katko/Maier, MMR 2009, 306, 307.

783 Vgl. Wandtke/Bullinger/Grützmacher, § 69g UrhG, Rn. 5; Dreier/Schulze/Dreier, § 69a UrhG, Rn. 17; Schricker/Katzenberger/Reber, $\S 95$ UrhG, Rn. 7; Dreyer/Kotthoff/Meckel/Hentsch/Dreyer, § 2 UrhG, Rn. 276; Kreutzer, CR 2007, 1, 2.

BGH, GRUR 2013, 370, 370; BayObLG, GRUR 1992, 508, 508 - Verwertung von Computerspielen; OLG Köln, CR 1992, 150, 151; OLG Karlsruhe, CR 1986, 723, 725; Wandtke/Bullinger/Bullinger, § 2 UrhG, Rn. 129; Fromm/Nordemann/Nordemann, § 2 UrhG, Rn. 204; Schricker/Loewenheim, § 2 UrhG, Rn. 217; Dreyer/Kotthoff/Meckel/Hentsch/Dreyer, § 2 UrhG, Rn. 276; Rauda, Computerspiele, Rn. 98; Förster, in Duisberg/Picot, Recht der Computer- und Videospiele, Kap. 2, Rn. 5; a. A. Schack, Urheberrecht, Rn. 731.

785 Schricker/Loewenheim/Spindler, § 69a UrhG, Rn. 27; Wandtke/Bullinger/Bullinger, § 2 UrhG, Rn. 130; Bullinger/Czychowski, GRUR 2011, 19, 21; Rauda, Computerspiele, Rn. 72. 
hinaus auch die einzelnen audiovisuellen Spielbestandteile wie die Musik des Spiels oder die im Spiel vorkommenden Spielfiguren stehen. ${ }^{786}$

Die Nutzung eines clientbasierten digitalen Spiels erfordert in der Regel sowohl eine dauerhafte Installation der Spielsoftware auf der Festplatte des Nutzers als auch eine vorübergehende Kopie derselben in den Arbeitsspeicher des Endgeräts. ${ }^{787}$ Sowohl bei der dauerhaften Kopie der Software auf der Festplatte ${ }^{788}$ als auch bei ihrer vorübergehenden Speicherung in den Arbeitsspeicher ${ }^{789}$ handelt es sich nach h. M. um eine Vervielfältigung gemäß $§ 16 \mathrm{Abs} .1 \mathrm{bzw}$. $\S 69 \mathrm{c} \mathrm{Nr} .1 \mathrm{UrhG}$.

Es werden in der Regel aber nicht nur die Software des digitalen Spiels, sondern auch die audiovisuellen Bestandteile auf die Festplatte bzw. in den Arbeitsspeicher des Endgeräts kopiert, sodass auch diese Schutzgegenstände im Sinne des § 16 Abs. 1 UrhG vervielfältigt werden. ${ }^{790}$ Die bloße Anzeige der geschützten Werke auf dem Bildschirm stellt dagegen keine urheberrechtlich relevante Nutzungshandlung dar. ${ }^{791}$

\section{Die Nutzungsberechtigung vor dem Supportende}

Um die aufgezählten Nutzungshandlungen vornehmen zu können, bedarf der Erwerber grundsätzlich einer Berechtigung.

\section{Einräumung von Nutzungsrechten}

Als eine solche Berechtigung kommt zunächst die Einräumung von Nutzungsrechten durch den Rechteinhaber nach $\S 31$ Abs. 1 Satz 1 UrhG in Betracht. Hierbei handelt es sich um eine dingliche Verfügung. ${ }^{792}$

Die Verpflichtung zur Einräumung der Nutzungsrechte entsteht in einem Kausalgeschäft, das - je nach vertraglicher Ausgestaltung - den im BGB geregelten Vertragstypen (z. B. Kauf, Werkvertrag oder Miete) zugeordnet werden kann. ${ }^{793}$ Dieses Kausalgeschäft ist vom Verpflichtungsgeschäft zu unterscheiden, in welchem die Pflicht des Publishers bzw. Händlers zur Überlassung der Spielkopie entsteht. ${ }^{794}$ Es spricht

786 BGH, GRUR 2017, 266, 269, Tz. 34 - World of Warcraft I; Förster, in Duisberg/Picot, Recht der Computer- und Videospiele, Kap. 2, Rn. 5; ausführlich dazu Rauda, Computerspiele, Rn. 82 ff.

Vgl. Psczolla, Onlinespielrecht, S. 86; BGH, CR 2011, 223, 224, Tz. 11-13 - Used Soft.

Wandtke/Bullinger/Grützmacher, § 69c UrhG, Rn. 4.

BGH, CR 2011, 223, 224, Tz. 13; GRUR 2017, 266, 269, Tz. 38; siehe ferner mit weiteren Nachweisen Dreier/Schulze/Schulze, § 16 UrhG, Rn. 13; Dreier/Schulze/Dreier, § 69c UrhG, Rn. 8.

790 Vgl. BGH, GRUR 2017, 266, 269, Tz. 38.

791 So ausdrücklich BGH, GRUR 2017, 266, 269, Tz. 38.

792 Loewenheim/Nordemann, in Loewenheim, Handbuch des Urheberrechts, § 26, Rn. 1; Scholz, ITRB 2012, 162, 163.

793 Loewenheim/Nordemann, in Loewenheim, Handbuch des Urheberrechts, § 26, Rn. 4.

794 Vgl. dazu Jaeger/Metzger, Open Source Software, Rn. 201. 
jedoch nichts dagegen, dass beide Kausalgeschäfte Teil eines einheitlichen Vertrages sein können.

Ebenfalls können die Verpflichtung zur Rechtseinräumung und ihre Erfüllung in der Praxis durchaus zusammenfallen. ${ }^{795}$ Entsprechend werden in der Regel Verpflichtungs- und Verfügungsgeschäft Teile eines einheitlichen Vertrages sein, sofern der Erwerber das digitale Spiel direkt vom Publisher erhält, sodass der Erwerber zusammen mit der Spielkopie auch die erforderlichen Nutzungsrechte erwirbt. Im Übrigen ist es nicht erforderlich, dass die Nutzungsrechtseinräumung ausdrücklich erfolgt. Nutzungsrechte an digitalen Spielen können nach der Rechtsprechung des BGH nämlich auch stillschweigend eingeräumt werden, wobei sich der Umfang der eingeräumten Nutzungsrechte dann nach dem von beiden Parteien zugrunde gelegten Vertragszweck gemäß $§ 31$ Abs. 5 Satz 1 UrhG bestimmt. ${ }^{796}$

Ausgehend von diesen Grundsätzen verpflichtet sich der Publisher sowohl im Falle des Direkt- als auch des Händlervertriebs im Überlassungsvertrag des Inklusivspiels zur Einräumung der erforderlichen Nutzungsrechte. Mit Blick auf den Händlervertrieb kommt in Analogie zum Abschluss des Supportvertrages ${ }^{797}$ das Kausalgeschäft dadurch zustande, dass der Erwerber das im Verkehrbringen des Inklusivspiels enthaltene konkludente Angebot des Publishers auf einen solchen Vertrag annimmt. Diese Verpflichtung tritt dabei neben die Obligation zur Überlassung des eigentlichen Clientprogramms und unterfällt wie diese den kaufvertraglichen Regelungen ( $\$ 453$ Abs. $1 \mathrm{BGB}){ }^{798}$

Entsprechend ist auch das Kausalgeschäft, welches den Publisher zur Einräumung von Nutzungsrechten in Bezug auf Konsolenspiele verpflichtet, als Kaufvertrag einzuordnen.

Auf das Kausalgeschäft, welches die Verpflichtung zur Rechtseinräumung in Bezug auf mietweise überlassene MMOG und Zeitspiele enthält, finden die mietvertragsrechtlichen Vorschriften entsprechend Anwendung.

Auf das der Einräumung von Nutzungsrechten an Free-to-Play-Spielen zugrunde liegende Kausalgeschäft können bei dauerhaft und kostenlos überlassener Software grundsätzlich die Vorschriften der Schenkung Anwendung finden. ${ }^{799}$

\section{Berechtigung gemäß § 69d Abs. 1 UrhG}

Darüber hinaus erhält der Erwerber von Software die notwendigen Nutzungsrechte nach herrschender Meinung gemäß $\S 69 \mathrm{~d}$ Abs. 1 UrhG auch ohne eine vertragliche

\footnotetext{
795 Loewenheim/Nordemann, in Loewenheim, Handbuch des Urheberrechts, § 26, Rn. 2.

796 Vgl. BGH, GRUR 2017, 266, 270, Tz. 43 f.

797 Siehe dazu oben, S. $61 \mathrm{f}$.

798 Siehe zur Typologisierung des Softwareüberlassungsvertrages oben, S. $65 \mathrm{ff}$.

799 Jaeger/Metzger, Open Source Software, Rn. 217; Koch, Computer-Vertragsrecht, S. 423.
} 
Nutzungsrechtseinräumung. ${ }^{800}$ Die Vorschrift erlaubt es dem „Berechtigten“, Vervielfältigungen i. S. d. § 69c Nr. 1, 2 UrhG für eine „,bestimmungsgemäße Benutzung“ des Computerprogramms vorzunehmen. „Berechtigter“ kann dabei der Käufer oder sonstige Erwerber sowie der Lizenznehmer des Computerprogramms sein. ${ }^{801}$ Was „,bestimmungsgemäße Benutzung“" eines Computerprogramms ist, richtet sich zuvörderst nach den Vereinbarungen zwischen Softwarehersteller und Erwerber des Programms, bei Fehlen solcher Vereinbarungen nach der Art und Ausgestaltung des Programms sowie seiner üblichen Nutzung. ${ }^{802}$

$\mathrm{Zu}$ beachten ist jedoch, dass sich die aus $\S 69 \mathrm{~d}$ Abs. 1 UrhG ergebende Nutzungsberechtigung nur auf das in dem digitalen Spiel enthaltene Computerprogramm, nicht jedoch auf die audiovisuellen und urheberrechtlich geschützten Spielbestandteile erstreckt. Die Regelungen der $\S \S 69$ a ff. UrhG sind auf Software zugeschnittene Sondervorschriften und auf andere Werkarten grundsätzlich nicht anwendbar. ${ }^{803}$ Hierfür erwirbt der Spieler also nicht automatisch Nutzungsrechte nach $\S 69 \mathrm{~d}$ Abs. 1 UrhG. Insofern bedarf es dann in jedem Fall einer rechtsgeschäftlichen Nutzungsrechtseinräumung.

Eine generelle Aussage darüber, wie der Erwerber die Nutzungsrechte bei den einzelnen digitalen Spielen erhält, kann nicht getroffen werden und ist von den Umständen des Einzelfalles sowie von der Art des digitalen Spiels abhängig. Für die Zwecke der weiteren Ausführungen wird davon ausgegangen, dass eine vertragliche Einräumung von Nutzungsrechten stattgefunden hat.

\section{Die Nutzungsberechtigung nach dem Supportende}

Fraglich ist, ob die Kündigung des Supports durch den Supportverpflichteten zugleich zu einer Beendigung der Nutzungsberechtigung des Erwerbers mit Blick auf das digitale Spiel führt.

Die Nutzungsrechtseinräumung stellt eine dingliche Verfügung dar, welche mit der Einräumung des Nutzungsrechts abgeschlossen ist und keiner fortdauernden Aktualisierung bedarf. ${ }^{804}$ Mangels Vorliegens eines Dauerschuldverhältnisses kommt damit auch weder eine ordentliche noch eine außerordentliche Kündigung der dinglichen

800 Dreier/Schulze/Dreier, § 69d UrhG, Rn. 2; Baus, MMR 2002, 14, 16; Blocher, in Walter/von Lewinski, European Copyright Law, Art. 5 Computer Program Directive, Rn. 5.5.12 f.; Hoeren, GRUR 2010, 665, 667; Köhler/Fritzsche, in Lehmann, Rechtsschutz und Verwertung von Computerprogrammen, 2. Teil, XIII, Rn. 47; Huppertz, CR 2006, 145, 146; vgl. EuGH, NJW 2012, 2565, 2569, Tz. 82 f. - UsedSoft; BGH, CR 2011, 223, 225, Tz. 22; CR 2014, 168, 170, Tz. 30 ff. - UsedSoft II; siehe für weitere Nachweise Wandtke/Bullinger/Grützmacher, § 69d UrhG, Rn. 26.

801 Vgl. Wandtke/Bullinger/Grützmacher, § 69d UrhG, Rn. 24 mwN.

802 Vgl. Schricker/Loewenheim/Spindler, § 69d UrhG, Rn. 7 f.

803 BGH, GRUR 2017, 266, 273, Tz. 66; GRUR 2016, 792, 797, Tz. 56 - Gesamtvertrag Unterhaltungselektronik.

804 Scholz, ITRB 2012, 162, 163; Stöckel/Brandi-Dohrn, CR 2011, 553, 556; vgl. BGH, GRUR 2009, 946, 948, Tz. 20 - Reifen Progressiv. 
Verfügung selbst in Betracht. ${ }^{805}$ Folglich ist lediglich eine Kündigung des der Verfügung zugrunde liegenden Verpflichtungsgeschäfts möglich ${ }^{806}$ Infolge der Kündigung dieses Schuldverhältnisses können die Nutzungsrechte wieder heimfallen. ${ }^{807}$

Grundsätzlich sind jedoch lediglich Dauerschuldverhältnisse wie z. B. Mietverträge kündbar. ${ }^{808}$ Austauschverhältnisse wie der Kaufvertrag können dagegen nicht gekündigt werden - jedenfalls kann ein ordentliches oder außerordentliches Kündigungsrecht hier nicht in Form von AGB gegenüber einem Verbraucher vereinbart werden, da dies den Erwerber der Software unangemessen benachteiligen würde ${ }^{809}$ Er erhielte nicht die für einen Kaufvertrag typische, uneingeschränkte Rechtsposition eines Eigentümers. ${ }^{810}$

Mit Blick auf Free-to-Play-Spiele und das als Schenkung zu typologisierende Kausalgeschäft, welches zur Rechtseinräumung verpflichtet, ist die Dauerhaftigkeit der Zuwendung ein wesentliches Typologisierungsmerkmal, mithilfe dessen die Schenkung von anderen unentgeltlichen Verträgen wie z. B. der Leihe abgegrenzt wird. ${ }^{811}$ Zwar schließt dies nicht aus, Klauseln zu vereinbaren, wonach im Falle der Verletzung der eingeräumten Nutzungsrechte durch den Erwerber ebendiese wieder automatisch zurückfallen können. ${ }^{812}$ Denn das Schenkungsrecht sieht in den $\S \S 528,530$ BGB unter bestimmten Umständen Rückforderungsansprüche bzw. sogar den Widerruf der Schenkung vor. ${ }^{813}$ Ein von etwaigen Verfehlungen des Beschenkten losgelöstes, formularmäßig vereinbartes ordentliches Kündigungsrecht des Schenkers widerspricht jedoch dem Erfordernis der Dauerhaftigkeit der Zuwendung des Vermögensbestandteils und stellt aus diesem Grunde eine überraschende (§ 305c Abs. 1 BGB) und den Erwerber unangemessen benachteiligende Klausel ( $\$ 307$ Abs. 2 Nr. 1 BGB) dar.

Das der Rechtseinräumung in Bezug auf Zeitspiele sowie mietweise überlassene MMOG zugrunde liegende Kausalgeschäft unterfällt demgegenüber den mietvertragsrechtlichen Regelungen und stellt daher ein Dauerschuldverhältnis dar. ${ }^{814}$ Wie bereits in Kapitel 3 dargestellt, endet der Support der Online-Komponenten für Zeitspiele und mietweise überlassende MMOG zulässigerweise mit der Kündigung des Mietvertrages, welcher die Überlassung des Spielclients zum Gegenstand hat. ${ }^{815}$ Entsprechend

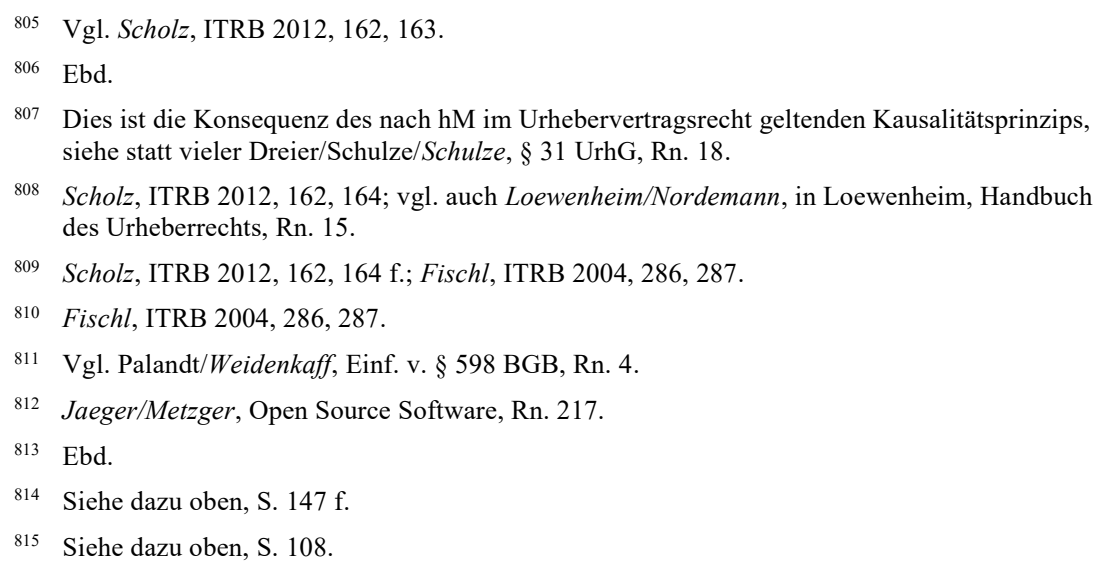


erlischt mit dem Ende des Mietverhältnisses bzgl. des Spielclients auch die Nutzungsberechtigung des Erwerbers durch Kündigung des mietvertragsrechtlichen Kausalgeschäfts, welches hier der Nutzungsrechtseinräumung zugrundeliegt.

Damit bleibt lediglich bei Inklusiv-, Konsolen- und Free-to-Play-Spielen die Berechtigung des Erwerbers zur Nutzung des digitalen Spiels samt Einzel- und OnlineMehrspielermodus auch nach der Kündigung des Supports der Online-Komponenten bestehen. Dies stellt den Rechteinhaber im Falle von Überschreitungen der Nutzungsrechte durch den Erwerber auch nicht schutzlos, da er Verletzungen seines Urheberrechts nach den $\S \S 97 \mathrm{ff}$. UrhG verfolgen kann. ${ }^{816}$

An diesem Ergebnis ändert sich auch nichts durch die Regelung des Art. 17 Abs. 1 der DI-RL. Diese Vorschrift untersagt dem Verbraucher nach der Beendigung des Vertrages über die Bereitstellung digitaler Inhalte die Nutzung derselben.

$\mathrm{Zu}$ beachten ist dabei zunächst, dass die Richtlinie nur solche Vertragsbeendigungen erfasst, welche vom Verbraucher ausgehen. ${ }^{817}$ Die Frage, ob ein Verbraucher einen digitalen Inhalt auch noch nach einer vom Unternehmer ausgesprochenen Kündigung weiternutzen darf, behandelt die Richtlinie damit nicht.

Darüber hinaus lässt die Richtlinie entsprechend Art. 3 Abs. 9 nationales sowie harmonisiertes Urheberrecht, insbesondere die Richtlinie 2001/29/EG unberührt. Mithin beurteilt sich die Frage der Berechtigung von Verbrauchern zur Nutzung solcher digitalen Inhalte, die Urheberrechtsschutz genießen, weiterhin nach nationalem bzw. harmonisiertem, europäischen Urheberrecht. Würde man die Regelung des Art. 17 Abs. 1 als ein uneingeschränktes Verbot der Nutzung erworbener digitaler Inhalte verstehen, würden schließlich auch die in der Richtlinie 2001/29/EG vorgesehenen und in nationales Recht umgesetzten Schranken leerlaufen.

\section{B. Rechtsschutz und Eliminierung von Online-DRM-Systemen nach dem Supportende}

Die Wiederherstellung der Nutzungsmöglichkeit von digitalen Spielen, deren Online-Komponenten keinen Support mehr erhalten, hat - je nachdem, welche Art von Online-Komponenten betroffen ist - eine andere Zielrichtung. ${ }^{818}$

So zielt die Wiederherstellung des Online-Mehrspielermodus auf die Rekonstruktion dieser Online-Komponente.

Sofern die Nutzungsmöglichkeit des digitalen Spiels aufgrund von supportlosen Online-DRM-Systemen eingeschränkt oder aufgehoben ist, zielt die Wiederherstellung der Nutzungsmöglichkeit in der Regel darauf ab, jegliche Abhängigkeit der Nutzungsmöglichkeit des Spiels von dieser Art Online-Komponente zu beseitigen. Durch permanente oder temporäre Eingriffe in den Programmcode der Spielkopie wird daher

\footnotetext{
816 Scholz, ITRB 2012, 162, 165.

817 Siehe dazu oben, S. $118 \mathrm{f}$.

818 Siehe dazu oben, S. 23.
} 
verhindert, dass die Kopie des digitalen Spiels zum Zweck der Authentifizierung der Spielkopie eine Verbindung mit einem DRM-Server aufbaut.

Zwar ist es darüber hinaus auch denkbar, einen privaten DRM-Server nachzubauen, jedoch erscheint dieser Weg aufgrund der damit verbundenen technischen Schwierigkeiten kaum durchführbar. ${ }^{819}$ Aus diesem Grund beschränken sich die folgenden Ausführungen auf die Eliminierung der Online-DRM-Systeme im Wege der Veränderung des Programmcodes der Spielkopie.

Die Online-DRM-Systeme schützen grundsätzlich sowohl die audiovisuellen Bestandteile des digitalen Spiels als auch die Steuerungssoftware desselben.

Im Folgenden soll untersucht werden, welchen Schutz supportlose Online-DRMSysteme genießen (I.) und ob dieser Schutz den soeben genannten Maßnahmen entgegensteht, welche auf die Beseitigung der Online-DRM-Systeme gerichtet sind (II.).

\section{Schutz supportloser Online-DRM-Systeme}

\section{Schutz gemäß § 95a Abs. 1, 2 UrhG}

Nach der Legaldefinition des $\S 95$ a Abs. 2 S. 1 UrhG handelt es sich bei technischen Maßnahmen um

„Technologien, Vorrichtungen und Bestandteile, die im normalen Betrieb dazu bestimmt sind, geschützte Werke oder andere nach diesem Gesetz geschützte Schutzgegenstände betreffende Handlungen, die vom Rechtsinhaber nicht genehmigt sind, zu verhindern oder einzuschränken ".

$\S 95$ a Abs. 1 UrhG statuiert ein Verbot der Umgehung solcher wirksamen technischen Maßnahmen.

Der aus Art. 6 Abs. 1 der InfoSoc-Richtlinie ${ }^{820}$ stammende Begriff der „technischen Maßnahmen" ist zum Zwecke eines hohen Schutzniveaus zugunsten der Rechtsinhaber weit auszulegen. ${ }^{821}$ Unerheblich ist daher, auf welche Art und Weise der Umgehungsschutz technologisch umgesetzt wird, also etwa mithilfe einer Verschlüsselung, mittels Passwörter oder durch Authentifizierungsprogramme. ${ }^{822}$ Der Begriff umfasst somit beispielsweise auch Maßnahmen, die einerseits im Träger des digitalen Spiels und andererseits in der zum Ablaufen des Spiels vorgesehenen Spielekonsole implementiert sind und zur Entfaltung ihres Schutzes eine Interaktion zwischen beiden Komponenten erfordern. ${ }^{823}$ Geschützt werden auch serverbasierte Zugangskontrollen, welche nur autorisierten Nutzern den Zugriff auf geschützte Inhalte im Wege der Vergabe von Benutzer- oder Gerätekennungen gestatten. ${ }^{824}$

\footnotetext{
819 Siehe dazu oben, S. 24 f.

820 RL 2001/29/EG.

EuGH, GRUR 2014, 255, 257, Tz. 27 - Nintendo Co., Ltd u. a. / PC Box Srl. u. a.

Vgl. Dreier/Schulze/Specht, § 95a UrhG, Rn. 14; siehe für eine Auflistung verschiedener Maßnahmen Fromm/Nordemann/Czychowski, § 95a UrhG, Rn. 15.

823 EuGH, GRUR 2014, 255, 257, Tz. 28.

824 Vgl. Dreyer/Kotthoff/Meckel/Hentsch/Dreyer, § 95a UrhG, Rn. 16.
} 
Die technischen Maßnahmen müssen außerdem objektiv (,im normalen Betrieb“) dazu bestimmt sein, zum Schutz urheberrechtlich geschützter Werke und Leistungen eingesetzt zu werden. ${ }^{825}$ Es genügt nicht, wenn sie diese Funktion lediglich beiläufig erfüllen. ${ }^{826}$

$\S 95$ a Abs. 2 Satz 2 UrhG kodifiziert außerdem das Erfordernis, dass die technische Maßnahme „wirksam“ ist. Dies meint nicht, dass der Mechanismus einen hundertprozentigen Umgehungsschutz bieten muss. ${ }^{827}$ Es kommt vielmehr darauf an, ob der durchschnittliche Nutzer die technische Maßnahme nicht ohne Weiteres überwinden kann. ${ }^{828}$

\section{a) Objektive Zweckbestimmung und Wirksamkeit der Online-DRM- Systeme}

Wie bereits dargestellt, kann die Beendigung des Supports von Online-DRM-Systemen dazu führen, dass die Nutzungsmöglichkeit des gesamten Spiels für alle Spieler wegfälltt. ${ }^{829}$ Es werden also nicht nur solche Spieler von der Nutzung ausgeschlossen, die beispielsweise eine Raubkopie verwenden oder aus anderen Gründen keine Nutzungsberechtigung haben, sondern auch diejenigen, die das Spiel ursprünglich legal erwarben und nutzten. Die supportlosen Online-DRM-Systeme können aufgrund der fehlenden Client-Server-Kommunikation keine Authentifizierung der Spielkopie mehr vornehmen.

Damit sind die Online-DRM-Systeme nunmehr in gewisser Weise funktionsgestört. Diese Funktionsgestörtheit allein führt jedoch nicht dazu, dass die supportlosen Online-DRM-Systeme aus dem Schutzbereich des § 95a Abs. 2 UrhG herausfallen. Funktionsgestörte Mechanismen können zwar dann aus dem Schutzbereich dieser Vorschrift auszunehmen sein, wenn sie den Schutz der Werke aufgrund einer Funktionsstörung nur zufällig bewirken und in Wirklichkeit einen ganz anderen Zweck verfolgen. ${ }^{830}$ Die supportlosen Online-DRM-Systeme sind aber nach wie vor noch im normalen Betrieb dazu bestimmt, urheberrechtlich relevante Handlungen wie z. B. die

825 Wandtke/Bullinger/Wandtke/Ohst, § 95a UrhG, Rn. 43; Fromm/Nordemann/Czychowski, § 95a UrhG, Rn. 12.

826 Fromm/Nordemann/Czychowski, § 95a UrhG, Rn. 12; Dreier/Schulze/Specht, § 95a UrhG, Rn. 14.

827 Vgl. OLG Hamburg, CR 2010, 45, 46.

828 OLG München, ZUM 2013, 806, 812; LG München, ZUM-RD 2013, 76, 79; OLG Hamburg, CR 2010, 45, 46; LG Frankfurt/M., MMR 2006, 766, 767; Arlt, in Hoeren/Sieber/Holznagel, Handbuch Multimedia-Recht, Teil 7.7, Rn. 21; Stickelbrock, GRUR 2004, 736, 738; Spindler/Schuster/Spindler, § 95a UrhG, Rn. 11; Dreyer/Kotthoff/Meckel/Hentsch/Dreyer, § 95a UrhG, Rn. 24; Wandtke/Bullinger/Wandtke/Ohst, § 95a UrhG, Rn. 50 mwN.

829 Siehe dazu die Einleitung.

830 Vgl. Wand, Technische Schutzmaßnahmen, S. 109; Peukert, in Loewenheim, Handbuch des Urheberrechts, § 34, Rn. 10. 
Vervielfältigung des Spiels auf der Festplatte oder im Arbeitsspeicher ${ }^{831}$ der Nutzerendgeräte zu verhindern oder einzuschränken. Die infolge der Supportbeendigung eingetretene Funktionsstörung ändert diesen Zweck nicht, sondern intensiviert vielmehr dessen Verwirklichung, indem die Online-DRM-Systeme nunmehr jeglichen $\mathrm{Zu}$ griff auf das Spiel verhindern.

Würde man die supportlosen Online-DRM-Systeme allein aufgrund dieser Funktionsstörung aus dem Schutzbereich des § 95a Abs. 2 UrhG herausnehmen, würde man dem von der InfoSoc-R1. erstrebten hohen Schutzniveau ${ }^{832}$ der Rechtsinhaber zuwiderlaufen. Außerdem würde - vor dem Supportende - jede noch so kurze Unterbrechung der Client-Server-Kommunikation genügen, um die Online-DRM-Systeme schutzlos zu stellen.

Darüber hinaus ist es grundsätzlich unerheblich, auf welchem technologischen Wege der Schutz der urheberrechtlich geschützten Werke und Leistungen erreicht wird. ${ }^{833}$ Denn schon der gezielte Einbau von Fehlern in Soft- oder Hardware, wie z. B. fehlerhafte Sektoren in DVDs, kann bereits als technische Maßnahme eingeordnet werden. ${ }^{834}$

Die objektive Zweckbestimmung der supportlosen Online-DRM-Systeme ist daher nach wie vor auf die Kontrolle urheberrechtlich relevanter Handlungen der digitalen Spiele gerichtet.

Die Maßnahmen werden darüber hinaus in der Regel auch als wirksam anzusehen sein. Zwar können die Online-DRM-Systeme - wie oben beschrieben - durch permanente oder temporäre Veränderungen des Programmcodes der Spielkopie eliminiert werden. ${ }^{835}$ Jedoch setzt diese Vorgehensweise ein nicht unerhebliches Spezialwissen über die angewandten Online-DRM-Systeme sowie über die Stellen innerhalb des Programmcodes voraus, an denen entsprechende Veränderungen vorgenommen werden müssen, um die Online-DRM-Systeme zu entfernen. Über ein solches Wissen werden in der Regel weder erfahrene Nutzer noch Durchschnittsspieler verfügen.

\section{b) Verhinderung nicht genehmigter Handlungen}

Die Definition technischer Maßnahmen gemäß § 95a Abs. 2 Satz 1 UrhG sowie i. S. d. Art. 6 Abs. 3 Satz 1 der InfoSoc-Rl. verlangt außerdem, dass die technischen Maßnahmen Handlungen einschränken oder verhindern müssen, die vom Rechtsinhaber nicht genehmigt worden sind.

Zunächst muss eine Genehmigung des Rechtsinhabers gemäß § 95a Abs. 2 Satz 1 UrhG mit Blick auf bestimmte urheberrechtlich relevante Nutzungshandlungen vorliegen. Unter einer Genehmigung gemäß $§ 95 a$ Abs. 2 Satz 1 UrhG ist sowohl die

831 Für die Einordnung des Ladens von Software in den Arbeitsspeicher als Vervielfältigung BGH, CR 2011, 223, 224.

Siehe dazu EuGH, GRUR 2014, 255, 257, Tz. 27.

833 Siehe dazu oben, S. 152.

834 Dreyer/Kotthoff/Meckel/Hentsch/Dreyer, § 95a UrhG, Rn. 17.

835 Siehe dazu oben, S. 24 f. 
vorherige als auch die nachträgliche Zustimmung i. S. d. § 183 BGB (Einwilligung) bzw. des $\S 184$ Abs. 1 BGB (Genehmigung) zu verstehen. ${ }^{836}$

Die Einwilligung und die Genehmigung schließen die Rechtswidrigkeit einer urheberrechtlichen Nutzungshandlung aus. ${ }^{837}$

Noch weiter geht die Einräumung urheberrechtlicher Nutzungsrechte, bei der schon der Tatbestand einer Verletzungshandlung entfällt. ${ }^{838}$ Darüber hinaus hat die Einräumung von Nutzungsrechten eine dingliche Wirkung und vermittelt eine im Grundsatz stärkere Rechtsposition als etwa die Einwilligung. ${ }^{839}$ Zwar wird die Einräumung von Nutzungsrechten in § 95a Abs. 2 Satz 1 UrhG nicht ausdrücklich erwähnt. Wenn aber schon eine lediglich schuldrechtliche Einwilligung für die Erfüllung des Tatbestands einer „Genehmigung“ gemäß § 95a Abs. 2 Satz 1 UrhG genügt, dann muss erst recht auch die dingliche Nutzungsrechtseinräumung als „Genehmigung“ ausreichen, da sie eine dingliche und damit noch stärkere Rechtsposition vermittelt.

Wie bereits dargestellt, wird der Erwerber des Spiels die Nutzungsrechte in der Regel vertraglich - entweder ausdrücklich oder konkludent - eingeräumt bekommen. ${ }^{840}$ In diesen Fällen liegt eine „Genehmigung“ für die konkreten Nutzungshandlungen, also z. B. das Speichern der audiovisuellen Bestandteile des Spiels auf der Festplatte oder im Arbeitsspeicher vor. Außerdem bleibt diese Genehmigung - zumindest bei Inklusiv-, Konsolen- und Free-to-Play-Spielen - auch nach dem Supportende noch bestehen. ${ }^{841}$

Die supportlosen Online-DRM-Systeme verhindern nun aber jegliche Nutzung des digitalen Spiels, also auch eine solche, für die der Rechtsinhaber an und für sich Nutzungsrechte erworben hat. Fraglich ist, ob die supportlosen technischen Maßnahmen aus diesem Grund ihren Schutz vor unerlaubten Umgehungen im Sinne des § 95a Abs. 1 UrhG verlieren.

Dies wäre die Konsequenz der Ansicht Peukerts, der dafür plädiert, den Rechtsschutz nicht nur für solche technischen Maßnahmen zu versagen, die urheberrechtlich irrelevante Nutzungshandlungen anbelangen, sondern auch für derartige Mechanismen, die eine vom Rechtsinhaber genehmigte Nutzungshandlung verhindern. ${ }^{842}$

Wenn man den Ansatz Peukerts konsequent zuende denkt, hätte dies zur Folge, dass vor allem technische Maßnahmen, die den Zugang zu geschützten Werken kontrollieren, weitgehend schutzlos gestellt werden. Derartige technische Maßnahmen blockieren nämlich zunächst und regulär auch für „legale“ Nutzer den Zugang und überprüfen

\footnotetext{
836 Wandtke/Bullinger/Wandtke/Ohst, § 95a UrhG, Rn. 42.

837 Fromm/Nordemann/Nordemann, § 97 UrhG, Rn. 24; vgl. BGH, GRUR 2010, 628, 631, Tz. 33 Vorschaubilder.

838 Fromm/Nordemann/Nordemann, § 97 UrhG, Rn. 24.

839 Vgl. Dreier/Schulze/Schulze, § 31 UrhG, Rn. 7.

840 Siehe dazu oben, S. $147 \mathrm{ff}$.

${ }^{841}$ Siehe dazu oben, S. $149 \mathrm{ff}$.

842 Peukert, in Loewenheim, Handbuch des Urheberrechts, § 34, Rn. 3, Fn. 4.
} 
ihre Berechtigung, bevor sie letztlich den Zugriff auf die geschützten Inhalte ermöglichen. ${ }^{843}$ Auf diese Weise funktionieren auch die in Kapitel 1 dargestellten OnlineDRM-Systeme ${ }^{844}$.

Dass die Ansicht Peukerts problematisch ist, wird auch mit Blick auf den Wortlaut des $\S 95 \mathrm{a}$ Abs. 2 Satz 1 UrhG bzw. des weitgehend wortgleichen Art. 6 Abs. 3 Satz 1 InfoSoc-Rl. deutlich. Die in diesen Vorschriften enthaltene Definition technischer Maßnahmen schreibt vor, dass solche Maßnahmen geschützt werden, die Nutzungshandlungen verhindern oder einschränken, „die vom Rechtsinhaber nicht genehmigt sind“. Der Wortlaut enthält also keine Einschränkung dahingehend, dass technische Maßnahmen nur so weit geschützt werden, als sie nicht genehmigte Handlungen kontrollieren. Daraus ist zu schließen, dass technische Maßnahmen auch dann Rechtsschutz genießen, wenn sie auch nicht genehmigte Nutzungshandlungen verhindern oder einschränken.

\section{c) Verhältnismäßigkeit}

Die Tatsache, dass die supportlosen Online-DRM-Systeme auch vom Rechtsinhaber genehmigte Nutzungshandlungen verhindern, könnte jedoch gegen ihre VerhältnismäBigkeit und damit gegen ihren Schutz nach § 95a Abs. 2 UrhG sprechen.

In seiner Nintendo-Entscheidung hatte sich der EuGH mit dem im Gesetz nicht ,angelegten ${ }^{\text {“845 }}$ Erfordernis der Verhältnismäßigkeit technischer Maßnahmen befasst. ${ }^{846}$ „Verhältnismäßigkeit" bedeutet hiernach, dass nur solche technischen Maßnahmen Rechtsschutz nach dem UrhG genießen, welche das Ziel verfolgen, die vom Rechtsinhaber nicht genehmigten Nutzungshandlungen zu verhindern. ${ }^{847}$ Die Maßnahmen müssen zur Verwirklichung dieses Ziels geeignet sein und dürfen nicht über das erforderliche Maß hinausgehen. ${ }^{848}$

Dies könnte man so verstehen, dass technische Maßnahmen bereits dann unverhältnismäßig sind, wenn sie vom Rechtsinhaber erlaubte Nutzungshandlungen verhindern. Allerdings kommt es für die Beurteilung der Verhältnismäßigkeit technischer Maßnahmen nicht darauf an, ob vom Rechtsinhaber genehmigte Nutzungshandlungen neben ungenehmigten verhindert oder beeinträchtigt werden, sondern ob Nutzungshandlungen ausgeschlossen werden, für die der Verwender der technischen Maßnahmen mangels Rechtsinhaberschaft überhaupt gar keine Genehmigung erteilen kann. Denn sowohl der EuGH als auch der BGH verdeutlichen, dass eine technische Maßnahme

${ }^{843}$ So kann z. B. die Eingabe eines Passworts erforderlich sein. Näher zu dieser Art von technischen Schutzmaßnahmen Fromm/Nordemann/Czychowski, § 95a UrhG, Rn. 15; Arlt, Digital Rights Management Systeme, S. 15.

844 Siehe dazu oben, S. 19 ff.

845 So Arlt, in Hoeren/Sieber/Holznagel, Handbuch Multimedia-Recht, Teil 7.7, Rn. 20.

846 EuGH, GRUR 2014, 255, 257, Tz. 30; daran anschließend BGH, MMR 2015, 460, 463 - Videospiel-Konsolen II.

${ }^{847}$ EuGH, GRUR 2014, 255, 257, Tz. 31; BGH, MMR 2015, 460, 463.

848 EuGH, GRUR 2014, 255, 257, Tz. 31; BGH, MMR 2015, 460, 463. 
nur dann unverhältnismäßig ist, wenn es andere Mechanismen gibt, die einen vergleichbaren Schutz bieten und Nutzungshandlungen, welche „keiner Genehmigung des Inhabers der Urheberrechte bed[ürfen] “ ${ }^{849}$, in geringerem Maße beeinträchtigten. Sowohl dem Fall des EuGH als auch dem des BGH lag nämlich eine technische Maßnahme zugrunde, die letztlich das „Abspielen zulässiger Drittentwicklungen“"850 auf einer Nintendo-Konsole und damit die Nutzung digitaler Spiele, welche nicht vom Publisher Nintendo vertrieben wurden, verhindern konnte.

Damit wird deutlich, dass die Verhinderung der vom Rechtsinhaber genehmigungsfähigen Nutzungen durch die supportlosen Online-DRM-Systeme nicht die Unverhältnismäßigkeit dieser technischen Maßnahmen begründen kann.

Insgesamt stellen die Online-DRM-Systeme somit auch in ihrem supportlosen $\mathrm{Zu}$ stand wirksame technische Maßnahmen gemäß § 95a Abs. 2 UrhG dar.

\section{Schutz gemäß $§ 69$ f Abs. 2 UrhG}

Es ist bereits dargestellt worden, dass Computerprogramme einem besonderen Rechtsschutz nach den $\S \S 69$ a ff. UrhG unterliegen. ${ }^{851}$ Gemäß $\S 69$ a Abs. 5 UrhG finden deshalb unter anderem die $\S \S 95$ a bis $95 \mathrm{~d}$ UrhG keine Anwendung auf Software.

$\S 69 f$ Abs. 2 UrhG räumt dem Rechtsinhaber ausdrücklich lediglich einen Anspruch auf Vernichtung von Mitteln ein, ,die allein dazu bestimmt sind, die unerlaubte Beseitigung oder Umgehung technischer Programmschutzmechanismen zu erleichtern“. Unter Programmschutzmechanismen sind alle Mittel zu verstehen, welche dazu dienen, Verletzungen des Urheberrechts am geschützten Programm zu verhindern ${ }^{852}$ Mithin sind auch supportlose Online-DRM-Systeme von diesem Begriff erfasst. Die objektive Zweckbestimmung der Online-DRM-Systeme ist auch nach dem Supportende nach wie vor auf die Verhinderung von Urheberrechtsverletzungen gerichtet.

Ein ausdrückliches Verbot der Umgehung von Programmschutzmechanismen findet sich in den $\S \S 69 \mathrm{a}$ ff. UrhG allerdings nicht. ${ }^{853} \mathrm{Ob}$ das UrhG dennoch über den $\S 69 \mathrm{f}$ Abs. 2 UrhG hinaus die Umgehung von Programmschutzmechanismen verbietet, ist umstritten und wurde in Literatur und Rechtsprechung bisher vor allem bei der eigenmächtigen Entfernung fehlerhafter Software-Dongle durch den Programmanwender diskutiert.

In den 1990er-Jahren war die durch Programmanwender eigenhändig vorgenommene Entfernung von sog. Software-Donglen Gegenstand mehrerer Gerichtsverfahren.

Bei diesen Donglen handelte es sich um Steckmodule, die auf die Parallele Schnittstelle des Anwender-PCs angebracht wurden und deren Vorhandensein ständig von

\footnotetext{
849 EuGH, GRUR 2014, 255, 257, Tz. 32; BGH, MMR 2015, 460, 463.

850 BGH, MMR 2015, 460, 462.

851 Siehe dazu oben, S. $146 \mathrm{ff}$.

852 Dreier/Schulze/Dreier, § 69f UrhG, Rn. 11.

853 Kreutzer, CR 2006, 804, 804; Jaeger, CR 2002, 309, 310; Mayer-Wegelin, JurPC 2009, WebDok 28, Abs. 81.
} 
einem Dongle-Abfrageprogramm überprüft wurde ${ }^{854}$ Für den Fall, dass der Dongle nicht mehr am PC angebracht oder defekt war, blockierte das Dongle-Abfrageprogramm den Ablauf der Software, zu deren Schutz der Rechtsinhaber den Dongle vorgesehen hatte ${ }^{855}$ Es handelte sich also um einen Schutzmechanismus, der verhindern sollte, dass unerlaubt hergestellte Kopien verwendet wurden, wenngleich die Herstellung solcher Kopien durch den Dongle nicht unmittelbar verhindert wurde. ${ }^{856}$

Gegenstand der erwähnten Gerichtsverfahren war die Frage, ob der Programmanwender im Falle eines Defekts des Dongles, welcher den Programmablauf behinderte, den Dongle eigenhändig entfernen durfte.

\section{a) Ansichten in Literatur und Rechtsprechung}

Einer Ansicht zufolge ist der Programmanwender dazu berechtigt, die softwareseitige Dongle-Abfrage in Fällen, in welchen der Dongle Störungen im Ablauf des Computerprogramms verursacht, eigenhändig und ohne Zustimmung des Rechtsinhabers zu entfernen. ${ }^{857}$

Diese Befugnis wird aus dem Recht des Programmanwenders zur „Fehlerberichtigung" nach § 69d Abs. 1 UrhG hergeleitet. Dagegen spreche auch nicht die Vorschrift des § 69f Abs. 2 UrhG. Diese Norm, welche auf § 69f Abs. 1 UrhG verweist, bezwecke zuvörderst die Bekämpfung der Herstellung und Verwendung von Raubkopien, wende sich aber nicht generell gegen die Umgehung von Programmschutzmechanismen. ${ }^{858}$ Die Verwendung des Wortes ,unerlaubt" in § 69f Abs. 2 UrhG mache außerdem deutlich, dass es nach dem Willen des Gesetzgebers auch erlaubte Umgehungen von Programmschutzmechanismen geben muss, wozu eben die Beseitigung von Programmschutzmechanismen zum Zwecke der Fehlerberichtigung gehöre. ${ }^{859}$

Würde man dem Programmanwender die Entfernung fehlerhafter Programmschutzmechanismen untersagen, würde dessen - vertraglich nicht abdingbares - Recht zur Erstellung einer Sicherungskopie ${ }^{860}$ und der damit verbundene Zweck der „Sicherung künftiger Benutzung“ unterlaufen, da der Programmanwender spätestens nach Ablauf

854 Für eine ausführliche Beschreibung der Funktionsweise von Donglen siehe König, NJW 1995, 3293, 3293.

König, NJW 1995, 3293, 3293.

Vgl. LG Düsseldorf, CR 1996, 737, 738.

857 LG Mannheim, NJW 1995, 3322, 3322 f. (aufgehoben durch OLG Karlsruhe CR 1996, 341); König, NJW 1995, 3293, 3294 f.; ders., NJW-CoR 1995, 191, 191; Dreyer/Kotthoff/Meckel/Hentsch/Kotthoff, § 69d UrhG, Rn. 7; Marly, NJW-CoR 1993, 21, 24; ders., JurPC 1992, 1652, 1673; Koch, NJW-CoR 1994, 293, 296; so auch für die Umgehung von Sperrmechanismen Jobke, Produktaktivierung, S. 191 f.; vgl. ferner Schack, Urheberrecht, Rn. 473, für den jedoch eine den Programmablauf behindernde Dongleabfrage schon dann fehlerhaft ist, wenn der Käufer der Software weder über das Vorhandensein noch die Bedeutung der Dongleabfrage aufgeklärt worden ist.

König, NJW 1995, 3293, 3294; ders., NJW-CoR 1995, 191, 191.

König, NJW 1995, 3293, 3295.

860 Siehe $\S 69$ d Abs. 2 UrhG. 
der Gewährleistungsfrist auch die hergestellten Sicherungskopien aufgrund des fehlerhaften Dongles nicht mehr verwenden könnte. ${ }^{861}$

Eine andere Ansicht in der Literatur und Rechtsprechung spricht sich gegen ein Selbsthilferecht des Softwareanwenders in Fällen von Störungen im Programmablauf aus, die durch einen fehlerhaften Dongle hervorgerufen werden. ${ }^{862}$ Gegen ein Selbsthilferecht des Softwareanwenders wendet diese Ansicht ein, dass nach der Konzeption des Herstellers des Programms die Dongle-Abfrage gerade Teil des Programms sei und damit auch zum fehlerfreien Betrieb der Software gehöre. ${ }^{863}$ Daher umfasse die bestimmungsgemäße Benutzung des Programms nach $\S 69 \mathrm{~d}$ Abs. 1 UrhG stets nur die Verwendung des Programms einschließlich des Dongles, sodass die Umgehung bzw. Beseitigung des Dongles dann aber auch keine zur bestimmungsgemäßen Programmnutzung notwendige Fehlerberichtigung darstellen könne. ${ }^{864}$ Die Ansicht, welche sich für ein Selbsthilferecht des Programmnutzers ausspricht, übersehe außerdem, dass mit der Umgehung gar keine Fehlerberichtigung des Dongles in Form einer Reparatur stattfinde, sondern der Dongle komplett entfernt werde, wodurch das Programm wesentlich umgestaltet werde. ${ }^{865}$

Es handle sich außerdem um Fehler, die allenfalls den Dongle bzw. sein Abfrageprogramm betreffen, nicht aber die Software selbst, sodass schon aus diesem Grunde zweifelhaft sei, ob überhaupt das Recht des Softwareanwenders zur Fehlerberichtigung ausgelöst wird. ${ }^{866} \mathrm{Zu}$ beachten sei schließlich, dass der Vertragspartner des Programmverwenders das Recht zur Fehlerbeseitigung - und damit auch ein etwaiges Recht zur Entfernung des fehlerhaften Dongles - vertraglich ausschließen könne. ${ }^{867}$

Eine vermittelnde Ansicht in der Literatur will dem Programmnutzer zwar ein Selbsthilferecht zur Umgehung des fehlerhaften Dongles gewähren, jedoch nur unter der Voraussetzung, dass der Programmhersteller bzw. Lieferant zur Beseitigung der Störung nicht bereit oder in der Lage ist. ${ }^{868}$ Insgesamt solle das Selbsthilferecht für

861 Vgl. König, NJW 1995, 3293, 3295.

862 OLG Karlsruhe, CR 1996, 341, 343; OLG Düsseldorf, CR 1997, 337, 339; OLG München, CR 1996, 11, 18; LG Düsseldorf, CR 1996, 737, 739; Raubenheimer, CR 1996, 343, 344; ders., CR 1996, 69, 72; Fromm/Nordemann/Czychowski, § 69d UrhG, Rn. 22; Möhring/Nicolini/Kaboth/Spies, § 69d UrhG, Rn. 8.

863 OLG Karlsruhe, CR 1996, 341, 343.

864 Vgl. ebd.; LG Düsseldorf, CR 1996, 737, 739; OLG Düsseldorf, CR 1997, 337, 339; Raubenheimer, CR 1996, 343, 344; Möhring/Nicolini/Kaboth/Spies, § 69d UrhG, Rn. 8.

865 LG Düsseldorf, CR 1996, 737, 739; OLG Düsseldorf, CR 1997, 337, 339.

866 Vgl. OLG München, CR 1996, 11, 18; Raubenheimer, CR 1996, 69, 73.

867 Raubenheimer, CR 1996, 69, 72.

868 Vgl. Schricker/Loewenheim/Spindler, § 69d UrhG, Rn. 11; Dreier/Schulze/Dreier, § 69d UrhG, Rn. 9; Baus, Verwendungsbeschränkungen, S. 193; Wandtke/Bullinger/Grützmacher, § 69d UrhG, Rn. 19; vgl. mit Blick auf Programmsperren Koch, CR 2002, 629, 635. 
den Nutzer nur als ultima ratio in Betracht kommen, sodass dem Hersteller bzw. Lieferant zuvor in jedem Fall die Möglichkeit zur Beseitigung des Fehlers gegeben werden müsse. ${ }^{869}$

\section{b) Stellungnahme}

Für die Frage, ob der Programmanwender eigenhändig einen defekten Programmschutzmechanismus entfernen darf, kann es zunächst nicht darauf ankommen, ob der Fehler dem Programm selbst immanent ist oder (nur) vom Programmschutzmechanismus ausgeht. Unter den Begriff des „Fehlers“ im Sinne des § 69d Abs. 1 UrhG fallen alle Elemente, die eine bestimmungsgemäße Benutzung des Programms einschränken. ${ }^{870}$ Dabei ist es unerheblich, ob diese Elemente Teil des Programms selbst sind oder von außerhalb kommen. ${ }^{871}$ Würden die durch externe Faktoren hervorgerufenen Fehler vom Recht zur Fehlerberichtigung ausgeschlossen werden, dürften auch Computerviren vom Programmanwender nicht beseitigt werden.

Zwar mag die bestimmungsgemäße Benutzung eines Programms auch den Einsatz bzw. das Vorhandensein eines Programmschutzmechanismus wie einen Dongle umfassen. Zur bestimmungsgemäßen Benutzung gehört aber auch und zuvörderst das „Laden, Anzeigen [und] Laufenlassen“ dieses Computerprogramms. ${ }^{872}$ Nur aufgrund der Erwartung, dass das Computerprogramm diese Funktionen auf seinem Endgerät ohne Probleme ausführen kann, hat der Anwender das Computerprogramm überhaupt erst erworben.

Würde man ein Recht des Erwerbers auf Entfernung des fehlerhaften Dongles verneinen, liefe das auf eine völlige Vernachlässigung der Interessen des Benutzers an einem funktionierenden Computerprogramm sowie auf einen übermäßigen Schutz der Interessen des Rechtsinhabers an einem intakten Dongle hinaus. Dies ist umso problematischer, wenn man bedenkt, dass die Vorschriften der $\S \S 69 \mathrm{a}$ ff. UrhG eben keinen ausdrücklichen Rechtsschutz vor Umgehung von Programmschutzmechanismen vorsehen ${ }^{873}$ Darüber hinaus darf die Fehlerberichtigung auch nicht generell vertraglich untersagt werden, da sie zu dem sog. abredefesten Kern der durch § 69d Abs. 1 UrhG vermittelten Nutzerrechte gehört. ${ }^{874}$ Eine Reparatur des Dongle-Abfrageprogramms oder des Dongles selbst dürfte dem Nutzer oftmals nicht möglich sein, weshalb wohl regelmäßig auch nur eine Entfernung des Dongles bzw. der Dongle-Anfrage in Frage kommen dürfte. ${ }^{875}$

\footnotetext{
869 Vgl. Baus, Verwendungsbeschränkungen, S. 193.

870 Schricker/Loewenheim/Spindler, § 69d UrhG, Rn. 10; Dreier/Schulze/Dreier, § 69d UrhG, Rn. 9.

871 Schricker/Loewenheim/Spindler, § 69d UrhG, Rn. 10.

872 Vgl. Dreier/Schulze/Dreier, §69d UrhG, Rn. 8.

873 Dazu Kreutzer, CR 2006, 804, 808.

874 BGH, NJW 2000, 3212, 3214 - Programmfehlerbeseitigung; Lehmann, NJW 1993, 1822, 1824; Dreier/Schulze/Dreier, $\S 69 \mathrm{~d}$ UrhG, Rn. 12. Siehe auch Erwägungsgrund 13 der RL 2009/24/EG.

875 Vgl. Wandtke/Bullinger/Grützmacher, § 69d UrhG, Rn. 19.
} 
Um jedoch dem Interesse des Rechtsinhabers an einer Verhinderung von Raubkopien Rechnung zu tragen, sollte - im Sinne der oben dargestellten vermittelnden Ansicht - die Umgehung nur dann zulässig sein, wenn weder der Rechtsinhaber noch der Lieferant oder Hersteller der Software zur Beseitigung des Fehlers bereit oder in der Lage ist. Diese Einschränkung ergibt sich aber schon aus dem Wortlaut des $\S 69 \mathrm{~d}$ Abs. 1 UrhG, nach dem Handlungen gemäß § 69c Nr. 1, 2 UrhG nicht der Zustimmung des Rechtsinhabers bedürfen, wenn sie für eine bestimmungsgemäße Benutzung des Programms ,notwendig“ sind. Insgesamt ist damit der vermittelnden Ansicht zu folgen.

\section{Schutz nach dem Zugangskontrolldiensteschutzgesetz (ZKDSG)}

Das am 23.03.2002 in Kraft getretene ZKDSG stellt sog. Zugangskontrolldienste sowie zugangskontrollierte Dienste unter einen besonderen rechtlichen Schutz vor unerlaubten Eingriffen und wurde zur Umsetzung der Richtlinie 98/84/EG (sog. Zugangskontrolldienste-Richtlinie) erlassen. ${ }^{876}$

Gemäß $§ 2$ Nr. 1 ZKDSG handelt es sich bei „,zugangskontrollierten Diensten“ um Rundfunkdarbietungen gemäß $\S 2$ des Rundfunkstaatsvertrages (RStV) bzw. um Telemedien gemäß $\S 1$ des Telemediengesetzes (TMG), die unter der Voraussetzung eines Entgelts erbracht und nur unter Verwendung eines Zugangskontrolldienstes genutzt werden können.

Die Beendigung des Supports der Online-DRM-Systeme führt dazu, dass offline nutzbare Spielkomponenten wie der Einzelspielermodus nicht mehr verwendet werden können.

Bei diesen Komponenten des digitalen Spiels handelt es sich jedoch regelmäßig nicht um Telemedien gemäß $§ 1$ Abs. 1 TMG. Hierunter fallen elektronische Informations- und Kommunikationsdienste, welche nicht ausschließlich Telekommunikationsbzw- telekommunikationsgestützte Dienste oder Rundfunk darstellen. ${ }^{877}$ Die als Telemedien einzuordnenden Informations- und Kommunikationsdienste werden mithilfe von Telekommunikationsmitteln erbracht ${ }^{878}$, weshalb Offline-Medien wie CDs und DVDs vom Telemedienbegriff ausgeschlossen sind ${ }^{879}$. Typische Telemediendienste stellen Online-Angebote wie Online-Zeitungen, Chat-Rooms, Internetsuchmaschinen, aber auch Werbe-E-Mails dar. ${ }^{880}$

Die Offline-Komponenten digitaler Spiele können grundsätzlich unabhängig von einer Verbindung mit einem Server genutzt werden. Ihre Nutzungsmöglichkeit ist damit nicht auf einen Online-Diensteanbieter und damit auch nicht auf Telekommunikationsmittel angewiesen. So wie klassische Offline-Medien stellen damit auch die offline

876 Federrath, in Dressel/Scheffler, Rechtsschutz gegen Dienstepiraterie, S. 1; Arlt, in Hoeren/Sieber/Holznagel, Handbuch Multimedia-Recht, Teil 7.7, Rn. 78; Bär/Hoffmann, MMR 2002, 654, 654.

877 Holznagel, in Hoeren/Sieber/Holznagel, Handbuch Multimedia-Recht, Teil 3, Rn. 87.

878 Ebd., Teil 3, Rn. 88.

879 Heckmann, in Heckmann, juris Praxiskommentar Internetrecht, Kap. 1, Rn. 37.

880 Holznagel, in Hoeren/Sieber/Holznagel, Handbuch Multimedia-Recht, Teil 3, Rn. 87. 
nutzbaren Spielkomponenten keine Telemedien dar. Da die Offline-Spielkomponenten somit nicht als Telemedien einzuordnen sind, können sie auch keine zugangskontrollierten Dienste im Sinne von $\S 2$ Nr. 1 ZKDSG darstellen.

Im Gegensatz zu den offline nutzbaren Spielkomponenten können Online-Komponenten, wie z. B. der Online-Mehrspielermodus, durchaus als Telemedien eingeordnet werden ${ }^{881}$ Dieser Dienst wird mithilfe von Telekommunikationsmitteln erbracht und erweitert darüber hinaus die inhaltlichen Nutzungsmöglichkeiten des Spiels durch die Zurverfügungstellung eines Masterservers, mit dem Spiele-Server angesteuert und zum Spielen ausgewählt werden können. Damit stellt der Online-Mehrspielermodus ein Telemedium i. S. d. § 1 Abs. 1 TMG dar.

Neben der Einordnung eines Dienstes als Telemedium ist eine weitere Voraussetzung für einen zugangskontrollierten Dienst, dass er auch unter der Voraussetzung eines Entgelts erbracht wird. Es muss also eine vermögenswerte Gegenleistung für die Dienstleistung erbracht werden. ${ }^{882}$ Wie bereits festgestellt, beinhaltet ein Teil der Gegenleistung des Käufers eines Inklusivspiels bereits die Vergütung für den Support der Online-Komponenten. Damit handelt es sich bei den online nutzbaren Spielkomponenten eines digitalen Spiels auch um einen Dienst, der unter Voraussetzung eines Entgelts erbracht wird.

Free-to-Play-Spiele fallen demgegenüber aus dem Begriff des zugangskontrollierten Dienstes heraus, da sämtliche Komponenten dieser Spiele - mit Ausnahme etwaiger virtueller Gegenstände - ohne eine vermögenswerte Gegenleistung erbracht werden.

Schließlich muss das Telemedium gemäß $\S 2 \mathrm{Nr} .1$ a. E. ZKDSG ,nur unter Verwendung eines Zugangskontrolldienstes genutzt werden können“. ,Zugangskontrolldienste" sind nach $\S 2$ Nr. 2 ZKDSG technische Verfahren oder Vorrichtungen, welche die erlaubte Nutzung eines zugangskontrollierten Dienstes ermöglichen.

Aus dem Erfordernis, dass durch den Zugangskontrolldienst erst die Nutzung des zugangskontrollierten Dienstes ermöglicht werden muss, folgert die Literatur, dass der Zugangskontrolldienst Verschlüsselungstechnologien einsetzen muss. ${ }^{883}$ Dafür spricht auch die Definition der "Zugangskontrollvorrichtung“ in Art. 2 lit. c) der RL 98/84/EG, welche jedes Gerät oder jedes Computerprogramm meint, das dazu bestimmt oder entsprechend angepasst ist, um den Zugang zu einem geschützten Dienst in verständlicher Form zu ermöglichen. ${ }^{884}$

Es ist allerdings zweifelhaft, ob die Online-DRM-Systeme das gesamte Spiel oder nur die Online-Komponenten von Inklusivspielen in dieser Form verschlüsseln. Doch selbst wenn das im Einzelfall gegeben sein sollte, muss beachtet werden, dass die Verschlüsselung des Dienstes gerade mit dem Zweck erfolgen muss, die Erzielung eines

881 So auch Baumann/Hofmann, ZUM 2010, 863, 868; siehe zur alten Rechtslage nach dem TDG auch Strobel, in Dressel/Scheffler, Rechtsschutz gegen Dienstepiraterie, S. 97 f.

882 Ausführlich dazu Strobel, in Dressel/Scheffler, Rechtsschutz gegen Dienstepiraterie, S. $101 \mathrm{f}$.

883 Ebd., S. 110.

884 Siehe ferner die Gesetzesbegründung, die vom Vorliegen eines „,unverständlichen“ Angebots ausgeht, welches durch den Zugangskontrolldienst wieder in „verständliche Form“ gebracht werden könne, was bspw. ein Decoder bewerkstelligen könne, BT-Drs. 14/7229, S. 7. 
Entgelts zu ermöglichen bzw. sicherzustellen. ${ }^{885}$ Es reicht dagegen nicht aus, dass Dienste aus Gründen der Sicherheit, der Authentifizierung oder des Kopierschutzes verschlüsselt werden. ${ }^{886}$ Wie oben ausgeführt, dienen die Online-DRM-Systeme aber nur der Authentifzierung des Spielers bzw. der Spielkopie. ${ }^{887}$ Damit stellen OnlineDRM-Systeme digitaler Spiele in der Regel keine Zugangskontrolldienste im Sinne des $\S 2$ Nr. 2 ZKDSG dar.

\section{Eliminierung supportloser Online-DRM-Systeme}

Nachdem zuvor der Rechtsschutz supportloser Online-DRM-Systeme festgestellt wurde, ist nunmehr zu untersuchen, ob dieser Schutz der Eliminierung supportloser Online-DRM-Systeme zur Wiederherstellung der Nutzungsmöglichkeit des digitalen Spiels entgegensteht.

\section{Bewertung nach § 95a Abs. 1, 3 UrhG}

\section{a) Eliminierung als Umgehung i.S.d. § 95a Abs. 1 UrhG}

Wie unter I. 1. festgestellt, genießen die supportlosen Online-DRM-Systeme als technische Maßnahmen gemäß § 95a Abs. 2 UrhG Schutz. ${ }^{888}$ Fraglich ist, ob die Eliminierung der supportlosen Online-DRM-Systeme eine Umgehung gemäß § 95a Abs. 1 UrhG darstellt.

Der Umgehungstatbestand setzt in objektiver Hinsicht voraus, dass die technische Maßnahme außer Kraft gesetzt oder abgeschwächt wird. ${ }^{889}$ Dabei kommt es nicht darauf an, auf welche Art und Weise die Umgehung erfolgt. ${ }^{890}$

In subjektiver Hinsicht muss dem Handelnden entsprechend dem Gesetzeswortlaut bekannt oder den Umständen nach bekannt sein, dass die Umgehung erfolgt, „um den Zugang zu einem solchen Werk oder Schutzgegenstand oder deren Nutzung zu ermöglichen“ ${ }^{891}$ Erforderlich ist also die Kenntnis des Umgehenden davon, dass die Umgehung mit dem Ziel erfolgt, das Werk bzw. den geschützten Gegenstand zu nutzen. ${ }^{892}$

Nach diesen Grundsätzen ist die von den Inhabern der Spielkopie vorgenommene Eliminierung der Online-DRM-Systeme durch Veränderung der Programmcodes der Spielkopie grundsätzlich als Umgehung dieser technischen Maßnahmen anzusehen.

\footnotetext{
885 Strobel, in Dressel/Scheffler, Rechtsschutz gegen Dienstepiraterie, S. 103 mit Verweis auf die Gesetzesbegründung, BT-Drs. 14/7229, S. 7. Siehe auch Erwg. 4 der RL 98/84/EG.

886 BT-Drs. 14/7229, S. 7.

887 Siehe dazu oben, S. $19 \mathrm{ff}$.

888 Siehe dazu oben, S. $152 \mathrm{ff}$.

889 Dreyer/Kotthoff/Meckel/Hentsch/Dreyer, § 95a UrhG, Rn. 31; Peukert, in Loewenheim, Handbuch des Urheberrechts, Rn. 15; Schricker/Götting, § 95a UrhG, Rn. 10.

890 Peukert, in Loewenheim, Handbuch des Urheberrechts, Rn. 15.

891 § 95a Abs. 1 2. Hs. UrhG.

892 Dreyer/Kotthoff/Meckel/Hentsch/Dreyer, $\S 95$ a UrhG, Rn. 30.
} 
Die Umgehung erfolgt hier gerade mit dem Ziel, das Spiel wieder bzw. weiternutzen zu können.

Es ist in der Einleitung dieser Arbeit erläutert worden, dass nicht nur private Spieler ein Interesse an der Wiederherstellung der Nutzungsmöglichkeit supportloser digitaler Spiele haben. Digitale Spiele sind darüber hinaus auch Gegenstand einer Vielzahl von wissenschaftlichen Disziplinen. Für die Wissenschaftler ist dabei in gleichem Maße wie für Spieler ein ungehinderter Zugriff auf alle Funktionen der digitalen Spiele erforderlich, welcher jedoch durch die supportlosen Online-Komponenten beschränkt bzw. unmöglich gemacht wird. Die Beendigung des Supports der Online-Komponenten behindert darüber hinaus aber auch Gedächtnisinstitutionen bei ihrer Arbeit, digitale Spiele zu erhalten und auszustellen. Vor diesem Hintergrund führen Erwägungsgrund 48 der InfoSoc-Rl. und daran anschließend auch die Gesetzesbegründung des deutschen Gesetzgebers zum Umsetzungsgesetz ${ }^{893}$ aus, dass Umgehungshandlungen, die (ausschließlich) Forschungszwecken dienen, nicht nach Art. 6 Abs. 1 InfoSoc-Rl. bzw. § 95a Abs. 1 UrhG verboten sein sollen. Beispielhaft werden hierfür Forschungsarbeiten im Bereich der Verschlüsselungstechniken genannt.

Diese Bereichsausnahme ist jedoch eng und umfasst nicht solche Umgehungsmaßnahmen, welche auf die - urheberrechtlich relevante - Nutzung der digitalen Spiele gerichtet sind ${ }^{894}$ Lediglich solche Forschungsvorhaben sind von dem Umgehungsverbot ausgenommen, die sich ausschließlich auf die Umgehung von technischen Maßnahmen beschränken. ${ }^{895}$

Aus diesem Grund hilft die Bereichsausnahme Wissenschaftlern, welche digitale Spiele selbst erforschen wollen, und Gedächtnisinstitutionen, die diese auszustellen gedenken, nicht weiter. Umgehungen, die zu diesen Zwecken vorgenommen werden, bleiben nach $\S 95 \mathrm{a}$ Abs. 1 UrhG untersagt.

\section{b) Verbot von Vorbereitungshandlungen gemäß § 95a Abs. 3 UrhG}

Die Umgehung der supportlosen Online-DRM-Systeme setzt ein nicht unerhebliches Fachwissen voraus, worüber der durchschnittliche Inhaber eines digitalen Spiels regelmäßig nicht verfügt. Er ist daher auf Hilfestellungen und Werkzeuge anderer angewiesen, mithilfe derer er die Umgehung der supportlosen Online-DRM-Systeme auch selbst vornehmen kann.

Wie bereits dargestellt, können derartige Hilfestellungen und Werkzeuge im Internet auf einschlägigen Webseiten von Fans, die sich der Wiederherstellung der Nutzungsmöglichkeit von digitalen Spielen verschrieben haben, ausfindig gemacht werden. ${ }^{896}$ Dazu gehören Anleitungen zur Wiedernutzbarmachung des digitalen Spiels sowie

\footnotetext{
893 Siehe BT-Drs. 15/38, S. 26.

894 Vgl. Dreyer/Kotthoff/Meckel/Hentsch/Dreyer, § 95a UrhG, Rn. 34.

895 Ebd., Rn. 38

896 Siehe dazu oben, S. $30 \mathrm{ff}$.
} 
Software, die heruntergeladen werden kann und die notwendigen Änderungen an der Spielkopie automatisch vornimmt. ${ }^{897}$

Die Produktion und Zurverfügungstellung solcher Werkzeuge und Hilfestellungen fallen in der Regel unter das Verbot von Vorbereitungshandlungen gemäß § 95a Abs. 3 Nr. 3 UrhG.

Software, welche automatisch die Spielkopie so verändern kann, dass supportlose Online-DRM-Systeme umgangen werden, wird unter die Begriffe der Vorrichtungen, Erzeugnisse oder Bestandteile zu fassen sein. ${ }^{898}$ Diese Software wird durch ihre Veröffentlichung auf den Fan-Webseiten auch ,,verbreitet“ im Sinne von $\S$ 95a Abs. 3 UrhG. Die Verbreitung im Sinne dieser Norm ist nicht in gleicher Weise auszulegen wie der gleichnamige Begriff in $\S 17$ UrhG. ${ }^{899}$ Von diesem Begriff wird vielmehr auch die Bereitstellung von Software im Internet zur Umgehung technischer Maßnahmen erfasst. ${ }^{900}$

Die Zurverfügungstellung von Anleitungen wird darüber hinaus regelmäßig auch eine Erbringung von Dienstleistungen gemäß § 95a Abs. 3 1. Halbsatz UrhG darstellen. ${ }^{901}$ Zwar ist umstritten, ob die „Erbringung von Dienstleistungen“ auch auf Handlungen Privater Anwendung findet. ${ }^{902} \mathrm{Im}$ Wortlaut der Vorschrift finden sich jedoch keine Anhaltspunkte dafür, Handlungen Privater vom Anwendungsbereich auszuschließen. Dies wird vor allem daran ersichtlich, dass der Besitz von Vorrichtungen, Erzeugnissen oder Bestandteilen nur dann verboten ist, wenn dieser gewerblichen Zwecken dient. Eine derartige Qualifikation haben die anderen Tatbestandsmerkmale und damit auch die Erbringung von Dienstleistungen im Tatbestand nicht erhalten. ${ }^{903}$ Damit ist auch der Austausch von Informationen zwischen Privaten erfasst. ${ }^{904}$

Eine weitere Voraussetzung ist, dass die Umgehungssoftware bzw. die Anleitung einen der drei alternativ genannten Zwecke in $\S 95$ a Abs. 3 Nr. 1 bis 3 UrhG erfüllen müssen. Die Umgehungssoftware und die Anleitung zur Umgehung werden regelmäBig hauptsächlich hergestellt bzw. erbracht, um die Umgehung wirksamer technischer Maßnahmen zu ermöglichen oder zu erleichtern i. S. d. § 95a Abs. 3 Nr. 3 UrhG.

${ }^{897}$ Siehe dazu oben, S. $30 \mathrm{ff}$.

898 Vgl. Peukert, in Loewenheim, Handbuch des Urheberrechts, Rn. 20; Schricker/Götting, § 95a UrhG, Rn. 25.

899 BT-Drs. 15/38, S. 26.

900 Dreyer/Kotthoff/Meckel/Hentsch/Dreyer, $\S 95$ a UrhG, Rn. 67.

901 BT-Drs. 15/38, S. 26; Dreier/Schulze/Specht, § 95a UrhG, Rn. 18.

902 Dagegen Schricker/Götting, § 95a UrhG, Rn. 31; Dreier/Schulze/Specht, § 95a UrhG, Rn. 18; Wandtke/Bullinger/Wandtke/Ohst, § 95a UrhG, Rn. 80.

903 BGH, ZUM 2008, 781, 783, Tz. 22 - Clone-CD; Pleister/Ruttig, MMR 2003, 763, 764; so auch in Bezug auf Art. 6 Abs. 2 der InfoSoc-RL Spindler, GRUR 2002, 105, 116.

904 Vgl. Peukert, in Loewenheim, Handbuch des Urheberrechts, Rn. 18; Fromm/Nordemann/Czychowski, § 95a UrhG, Rn. 47. 


\section{c) Zwischenergebnis}

Die Online-DRM-Systeme genießen auch in ihrem supportlosen Zustand Schutz nach § 95a UrhG. Dieser Schutz hat zur Folge, dass der Nutzer die technischen Maßnahmen nicht umgehen darf ( $\$ 95$ a Abs. 1 UrhG), auch wenn der Support bereits beendet worden ist und er noch immer zur Nutzung der Spielkopie berechtigt ist.

Darüber hinaus können auch Vorbereitungsmaßnahmen wie die Zurverfügungstellung von Anleitungen zur Umgehung der technischen Maßnahmen im Internet oder die Herstellung und Verfügbarmachung von Umgehungssoftware unter das Verbot des $\S 95$ a Abs. 3 UrhG fallen.

\section{Bewertung nach § 69d Abs. 1 UrhG}

Es ist bereits herausgearbeitet worden, dass die Umgehung von Programmschutzmechanismen im Urheberrecht für Computerprogramme nicht uneingeschränkt erlaubt ist. Die Umgehung ist allerdings dann zulässig, wenn weder der Rechtsinhaber noch der Lieferant oder Hersteller der Software zur Beseitigung des gemäß $\S 69 \mathrm{~d}$ Abs. 1 UrhG fehlerhaften Programmschutzmechanismus bereit oder in der Lage ist. ${ }^{905}$

Mit Blick auf die supportlosen Online-DRM-Systeme stellt sich dabei die Frage, ob die dauerhafte Unterbrechung der Client-Server-Verbindung infolge der Beendigung des Supports der Online-Komponenten als „Fehler“ i. S. d. § 69d Abs. 1 UrhG eingeordnet werden kann.

Eine Definition des Fehlerbegriffs findet sich weder im UrhG noch in der Computerprogramm-Rl.

Die Literatur versteht unter dem Begriff „Fehler“ diejenigen Elemente eines Programms, welche die bestimmungsgemäße Benutzung einschränken. ${ }^{906}$ Fehler können sowohl programminterne als auch externe Faktoren darstellen, wie z. B. Programmiermängel, Programmabstürze und Funktionsstörungen, aber auch Viren und Bugs, welche die Software „befallen“. 907 Vom Fehlerbegriff wird daher auch das gestörte Zusammenwirken des Programms mit einem Peripheriegerät angesehen. ${ }^{908}$ Der Fehler braucht darüber hinaus auch nicht bereits im Zeitpunkt des Erwerbs des Computerprogramms vorgelegen zu haben, sondern kann auch erst nachträglich auftreten. ${ }^{909}$

905 Siehe dazu oben, S. $160 \mathrm{ff}$.

906 Wand, Technische Schutzmaßnahmen, S. 128; Schricker/Loewenheim/Spindler, § 69d UrhG, Rn. 10; Dreier/Schulze/Dreier, § 69d UrhG, Rn. 9; Blocher, in Walter/von Lewinski, Europäisches Urheberrecht: Kommentar, Art. 5, Rn. 27; vgl. auch Dreier/Vogel, Software- und Computerrecht, S. 66.

907 Dreier/Schulze/Dreier, § 69d UrhG, Rn. 9; Schricker/Loewenheim/Spindler, § 69d UrhG, Rn. 10; Möhring/Nicolini/Kaboth/Spies, § 69d UrhG, Rn. 7; Ahlberg/Götting/dies., § 69d UrhG, Rn. 7; Dreier/Vogel, Software- und Computerrecht, S. 66; Geiger, Umarbeitungsrecht, S. 109; Blocher, in Walter/von Lewinski, Europäisches Urheberrecht: Kommentar, Art. 5, Rn. 27; Lehmann, NJW 1993, 1822, 1823.

So Dreier/Vogel, Software- und Computerrecht, S. 66.

909

Insofern unterscheidet sich der Fehlerbegriff nach $\S 69 \mathrm{~d}$ Abs. 1 UrhG vom Sachmangelbegriff gem. § 434 Abs. 1 Satz 1 BGB, Koch, CR 2002, 629, 635; vgl. Schricker/Loewenheim/Spindler, $\S 69 \mathrm{~d}$ UrhG, Rn. 10; Möhring/Nicolini/Kaboth/Spies, § 69d UrhG, Rn. 7. 
Nach einer Minderansicht in der Rechtsprechung werden vom Fehlerbegriff allerdings nur solche „Unzulänglichkeiten“ des Programms erfasst, welche der Hersteller des Programms nicht beabsichtigt habe. ${ }^{910}$ An dieser Ansicht ist jedoch problematisch, dass sich für den Anwender des Programms kaum zweifelsfrei feststellen lässt, welche „Unzulänglichkeiten“ des Programms sein Programmhersteller beabsichtigt hat und welche nicht. Außerdem könnte der Programmhersteller als Folge dieser Ansicht das Recht des Software-Anwenders zur Fehlerberichtigung beliebig einschränken, wenn nicht sogar vollständig ausschließen, indem er schlicht behauptet, (alle) Unzulänglichkeiten im Programm auch so beabsichtigt zu haben. Dass dies aber nicht richtig sein kann, belegt die Tatsache, dass die Fehlerberichtigung - wie bereits erläutert - zum abredefesten Kern der Nutzerbefugnisse gehört, die $\S 69 \mathrm{~d}$ Abs. 1 UrhG vorsieht.

Damit ist der Fehlerbegriff mit der überwiegenden Literaturansicht nicht subjektiv, sondern allein objektiv zu bestimmen, indem gefragt wird, ob ein Element vorliegt, welches die bestimmungsgemäße Benutzung des Programms beeinträchtigt.

Die endgültige Unterbrechung der Client-Server-Kommunikation infolge der Beendigung des Supports der Online-Komponenten führt zu einer Beeinträchtigung der bestimmungsgemäßen Benutzung des digitalen Spiels.

Die Beendigung des Betriebs der DRM-Server hat nämlich zur Folge, dass die Software des digitalen Spiels keine Verbindung mehr mit diesen Servern aufbauen und die erforderliche Authentifizierung nicht mehr durchführen kann. Zur bestimmungsgemäßen Benutzung des digitalen Spiels gehört es, dass der Erwerber dieses auf seinem Endgerät überhaupt ablaufen lassen kann. Diese Nutzungsmöglichkeit fällt aufgrund der soeben beschriebenen Funktionsstörung weg. Zwar liegt die Funktionsstörung in der Beendigung des Betriebs der DRM-Server begründet, jedoch lässt sich der Betrieb der DRM-Server von der Spielkopie funktionell nicht trennen.

Zwar ist die Beendigung des Supports der Online-Komponenten vom Publisher darüber hinaus beabsichtigt und die sich daraus ergebende Beeinträchtigung der Nutzungsmöglichkeit zumindest in Kauf genommen, jedoch führt diese Intention nicht dazu, dass die permanente Unterbrechung der Client-Server-Kommunikation vom Fehlerbegriff des $\S 69 \mathrm{~d}$ Abs. 1 UrhG auszuschließen ist.

Damit liegt ein Fehler im Sinne des $§ 69 \mathrm{~d}$ Abs. 1 UrhG vor. Sofern die Nutzungsberechtigung des Erwerbers mit Blick auf das Spiel noch nicht weggefallen ist ${ }^{911}$, kann der Spieler den Fehler des Programms durch Eliminierung der vom Programm durchgeführten Serveranfragen berichtigen. Allerdings gilt das Recht zur Fehlerberichtigung nur insoweit, als der Publisher nicht bereit oder in der Lage ist, den Fehler selbst zu beheben. Ein uneingeschränktes Recht zur Selbsthilfe würde die Interessen des Publishers an einem Schutz seiner Software vor unerlaubten Vervielfältigungen unangemessen beeinträchtigten.

\footnotetext{
910 So LG Düsseldorf, CR 1996, 737, 739.

911 Siehe dazu oben, S. 149 ff.
} 


\section{Verhältnis von $§ 95 a$ Abs. 1 und $\S$ 69a ff. UrhG}

Die Untersuchung der Frage, ob supportlose Online-DRM-Systeme zur Wiederherstellung der Nutzungsmöglichkeit digitaler Spiele eliminiert werden dürfen, hat ergeben, dass die Umgehung zwar nach § 95a Abs. 1 UrhG unzulässig, nach den Sondervorschriften der $\S \S 69 \mathrm{a}$ ff. UrhG für Computerprogramme jedoch unter bestimmten Umständen gestattet ist. Da die Online-DRM-Systeme jedoch grundsätzlich sowohl die audiovisuellen Bestandteile des digtialen Spiels als auch die Steuerungssoftware schützen, ist zu untersuchen, welche der beiden Regelungsregime Anwendung findet. Diese Frage ist in Literatur und Rechtsprechung stark umstritten.

\section{a) Schwerpunkttheorie}

Ein Teil der Literatur schlägt zur Lösung des soeben erläuterten Konflikts die sog. Schwerpunkttheorie vor. ${ }^{912}$ Demnach finden auf ein hybrides Werk diejenigen urheberrechtlichen Vorschriften Anwendung, welche für den prägenden Teil, also den Schwerpunkt des Werkes gelten. ${ }^{913}$ Dieser Schwerpunkt könne anhand des wirtschaftlichen Wertes der einzelnen Komponenten des Werkes ermittelt werden. ${ }^{914}$ Größtenteils sieht die Literatur den Schwerpunkt im Steuerungsprogramm der digitalen Spiele mit der Folge, dass die §§ 69a ff. UrhG und nicht die §§ 95a ff. UrhG auf das digitale Spiel und damit auch auf die technischen Maßnahmen bzw. Programmschutzmechanismen Anwendung finden. ${ }^{915}$ Dafür spreche, dass Kopierschutzmaßnahmen digitaler Spiele in der Regel nicht die Vervielfältigung von Bildschirmausgaben verhindern würden, sondern erst dann eingriffen, wenn die Software des Spiels kopiert wird. ${ }^{916}$ Außerdem sei es letztlich die Software des digitalen Spiels, welche das Spielerlebnis erst visualisiere. ${ }^{917}$

Für die Schwerpunkttheorie wird angeführt, dass durch sie eine einseitige Benachteiligung von Interessengruppen und eine künstliche Aufspaltung des hybriden Werkes in seine Einzelbestandteile sowie eine isolierte rechtliche Bewertung derselben vermieden werde. ${ }^{918}$ Schließlich sei der Gedanke, das anwendbare Recht anhand des

912 Kreutzer, CR 2007, 1, 4ff.; Redeker, IT-Recht, Rn. 125; Förster, in Duisberg/Picot, Recht der Computer- und Videospiele, Kap. 2, Rn. 28; Wandtke/Bullinger/Grützmacher, § 69a UrhG, Rn. 83; Schweyer, Reverse Engineering, S. 209 f.; Grützmacher, ITRB 2015, 120, 122;

Fromm/Nordemann/Czychowski, § 69a UrhG, Rn. 45; Schröder, MMR 2013, 80, 82; im Ergebnis auch Bullinger/Czychowski, GRUR 2011, 19, 25.

913 Vgl. Kreutzer, CR 2007, 1, 4.

914 Ebd., 4, 7; Wandtke/Bullinger/Grützmacher, § 69a UrhG, Rn. 83.

915 Kreutzer, CR 2007, 1, 7; Redeker, IT-Recht, Rn. 125; Förster, in Duisberg/Picot, Recht der Computer- und Videospiele, Kap. 2, Rn. 28; Wandtke/Bullinger/Grützmacher, § 69a UrhG, Rn. 83; Schröder, MMR 2013, 80, 82; a. A. Bullinger/Czychowski, GRUR 2011, $19,25$.

916 Förster, in Duisberg/Picot, Recht der Computer- und Videospiele, Kap. 2, Rn. 28.

917 Schröder, MMR 2013, 80, 82; so auch für auf Software basierende Multimedia-Anwendungen im Allgemeinen Koch, GRUR 1995, 459, 462.

918 Vgl. Kreutzer, CR 2007, 1, 6; Schröder, MMR 2013, 80, 82. 
Schwerpunktes des Regelungsgegenstandes zu ermitteln, dem Zivilrecht nicht fremd, da die Rechtsprechung in gleicher Weise auch bei gemischten Verträgen verfahre. ${ }^{919}$

\section{b) Trennungstheorie}

Der BGH und ein weiterer Teil der Literatur vertreten demgegenüber die sog. Trennungstheorie. Hiernach finden auf digitale Spiele als hybride Werkformen die Vorschriften der $\S \S 69 \mathrm{a}$ ff. UrhG und der $\S \S 95 \mathrm{a}$ ff. UrhG nebeneinander Anwendung. ${ }^{920}$ Letztlich trennt diese Auffassung also streng zwischen den audiovisuellen Bestandteilen und dem Steuerungsprogramm des digitalen Spiels.

Für diese Theorie wird ins Feld geführt, dass es nicht hinnehmbar sei, hybriden Werken wie digitalen Spielen den weitergehenden Schutz der InfoSoc-Rl. nur deshalb zu entziehen, weil die technischen Maßnahmen auch dem Schutz der Software des Spiels dienen. ${ }^{921}$ Anderenfalls würden komplexe Werke schlechter gestellt als Werke, die nicht aus verschiedenen Werkarten zusammengesetzt sind. ${ }^{922}$ Darüber hinaus würde der Schutz nach Art. 6 der InfoSoc-Rl. in unangemessner Weise abgeschwächt. ${ }^{923}$

Der BGH sieht sich in seiner Ansicht durch das Nintendo/PC Box-Urteil des EuGH $^{924}$ aus dem Jahre 2014 bestätigt. ${ }^{925}$ Das italienische Vorlageersuchen in diesem Fall ging aus einem Rechtsstreit zwischen dem Entwickler und Publisher Nintendo einerseits und dem Unternehmen PC Box andererseits hervor. PC Box hatte ohne Erlaubnis Spielekonsolen Nintendos derart modifiziert, dass auf ihnen - unter Umgehung technischer Maßnahmen - nachgeahmte Nintendo-Spiele sowie andere Software ohne Genehmigung von Nintendo ablaufen konnten. Diese Konsolen hatte PC Box an Endnutzer veräußert. Gegenstand des Vorlageverfahrens war zum einen die Frage, ob auch solche technischen Maßnahmen nach Art. 6 Abs. 3 der InfoSoc-Rl. Schutz genießen, die nicht nur im Träger des Videospiels, sondern auch in der Konsole, auf welcher das Spiel ablaufen soll, implementiert sind. ${ }^{926}$ Zum anderen wurde der EuGH gefragt, nach

919 Vgl. Schröder, MMR 2013, 80, 82.

920 BGH, GRUR 2013, 1035, 1037, Tz. 24 - Videospiel-Konsolen I; daran anschließend GRUR 2015, 672, 676, Tz. 44 - Videospiel-Konsolen II; GRUR 2017, 541, 542 - Videospiel-Konsolen III; dem BGH folgend Roth, MMR 2013, 671, 673; Arnold, GRUR-Prax 2013, 410, 410; Spindler/Schuster/Spindler, § 95a UrhG, Rn. 4; Ludwig/Falker, K\&R 2013, 654, 655; Hilgert, CR 2014, 354, 356; wohl auch Schricker/Loewenheim/Spindler, § 69a UrhG, Rn. 27; zuvor schon Katko/Maier, MMR 2009, 306, 306; Lindhorst, technische Maßnahmen, S. 144.

BGH, GRUR 2013, 1035, 1037, Tz. 24; Roth, MMR 2013, 671, 673; Spindler/Schuster/Spindler, § 95a UrhG, Rn. 4.

Hilgert, CR 2014, 354, 356; Arnold, GRUR-Prax 2013, 410, 410; Ludwig/Falker, K\&R 2013, 654, 655; vgl. Hermes, GRUR-Prax 2014, 37, 37.

Lindhorst, technische Maßnahmen, S. 144.

EuGH, GRUR 2014, 255, 255.

BGH, GRUR 2015, 672, 674 f., Tz. 43.

EuGH, GRUR 2014, 255, 256, Tz. 19. 
welchen Kriterien der Umfang des Rechtsschutzes technischer Maßnahmen gegen eine Umgehung gemäß Art. 6 der InfoSoc-R1. zu bestimmen sei. ${ }^{927}$

Der EuGH bejahte die erste Frage und führte zur Beantwortung der zweiten Frage aus, dass technische Maßnahmen den Grundsatz der Verhältnismäßigkeit wahren müssen. ${ }^{928}$ Im Rahmen seiner Ausführungen erläuterte der Gerichtshof außerdem, dass Videospiele nicht nur aus Computerprogrammen, sondern auch aus audiovisuellen Elementen bestehen. ${ }^{929}$ Diese Bestandteile unterliegen dem Schutz der InfoSoc-R1 $1{ }^{930}$ Dieser Richtlinie und Art. 1 Abs. 1 der RL 2009/24 EG lasse sich nicht entnehmen, dass das Gesamtwerk einer anderen Regelung unterliegen müsse als dessen Bestandteile. ${ }^{931}$ Das sich aus Computerprogrammen und audiovisuellen Bestandteilen ergebende Gesamtwerk unterfalle damit gleichfalls dem Schutz des Art. 6 der InfoSoc-R1. ${ }^{932}$

Neben dem BGH interpretieren auch Teile der Literatur die Entscheidung des EuGH dahingehend, dass Videospiele in ihrer Gesamtheit sowohl der InfoSoc- als auch der Computerprogramm-Rl. unterliegen. ${ }^{933}$ Eindeutig lässt sich diese Aussage der Entscheidung des EuGH jedoch nicht entnehmen. Es bleibt daher unklar, ob und in welchem Umfang tatsächlich auch die Computerprogramm-Rl. neben der InfoSoc-Rl. auf das Videospiel-Gesamtwerk Anwendung findet. ${ }^{934}$ Jedenfalls ist der Entscheidung des Gerichtshofs aber zu entnehmen, dass die InfoSoc-Rl. auf das digitale Spiel als Ganzes Anwendung findet und die technischen Maßnahmen des Spiels daher (auch) dem Schutz des Art. 6 der InfoSoc-Rl. unterfallen.

\section{c) Weitere Ansichten}

Darüber hinaus wird zum Teil argumentiert, dass $§ 69$ a Abs. 5 UrhG restriktiv auszulegen sei und nur auf Computerprogramme im eigentlichen Sinn bzw. auf solche Sachverhalte anzuwenden sei, in denen neben Software keine andere Werkart betroffen ist. ${ }^{935}$ Würde man in den Regelungsbereich des $\S 69 \mathrm{a}$ Abs. 5 UrhG auch Werke fallen lassen, die lediglich auf Computerprogrammen basieren, stehe eine „Durchlöcherung" des Schutzes nach den $\S \S 95$ a ff. UrhG zu befürchten. ${ }^{936}$

Andere wollen die Anwendung der $\S \S 69$ ff. UrhG bzw. der $\S \S 95 a$ ff. UrhG davon abhängig machen, ob das Computerprogramm Voraussetzung für die Visualisierung des audiovisuellen Inhalts ist oder ob für die Nutzung dieser Inhalte der Ablauf der

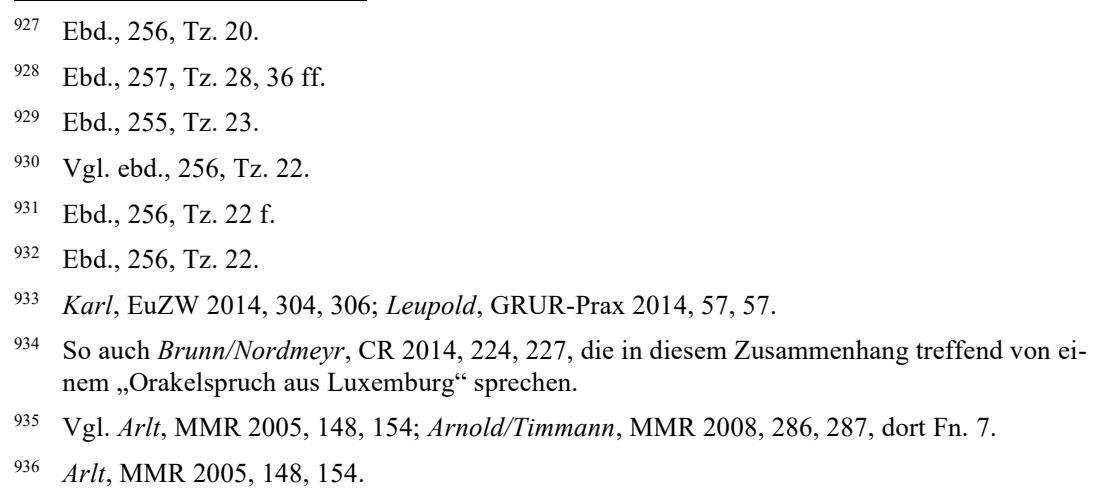


Software irrelevant sei. ${ }^{937}$ Dementsprechend sollen die $\S \S 69$ a ff. UrhG Anwendung finden, wenn der Ablauf der Software Voraussetzung für die Darstellung der audiovisuellen Inhalte ist.

\section{d) Stellungnahme}

Gegen die beiden zuletzt genannten Ansichten spricht, dass die Vorschriften der $\S \S$ 95a ff. UrhG auf digitale Spiele hiernach praktisch nie Anwendung finden würden, da davon auszugehen ist, dass die Wiedergabe der audiovisuellen Elemente dieser Spiele stets vom Ablauf der Software abhängig ist. Dies würde jedoch eine unangemessene Verkürzung des Schutzes technischer Maßnahmen für digitale Spiele bedeuten.

Gegen die Schwerpunkttheorie spricht die erhebliche Rechtsunsicherheit, die mit dieser Ansicht verbunden ist. ${ }^{938}$ Das Kriterium des wirtschaftlichen Schwerpunktes eines digitalen Spiels ist nämlich kaum dafür geeignet, die anwendbaren Rechtsvorschriften eindeutig zu bestimmen. So ist schon unklar, auf welche Perspektive zur Bestimmung des Schwerpunktes - die des Erwerbers oder die des Publishers? - abzustellen ist. Darüber hinaus ist es fraglich, ob bei digitalen Spielen ein solcher Schwerpunkt überhaupt existiert. Denn sowohl für Erwerber als auch für Publisher dürften alle Komponenten eines digitalen Spiels in gleichem Maße von hoher wirtschaftlicher Bedeutung sein. Das Spiel funktioniert schließlich nur zusammen mit all seinen Komponenten.

Für die Trennungstheorie spricht dagegen, dass sie beiden Regelungskomplexen die $\S \S 69$ a ff. UrhG sowie die $\S \S 95 \mathrm{a}$ ff. UrhG - Geltung verschafft und nicht einen zulasten des anderen ausschließt. Sie trägt der Tatsache in angemessener Weise Rechnung, dass digitale Spiele aus untrennbar miteinander verbundenen Komponenten bestehen, die alle für das Funktionieren des Spiels erforderlich und daher auch allesamt auf angemessenen rechtlichen Schutz angewiesen sind. Zwar führt die Trennungstheorie in der Konsequenz dazu, dass von beiden Regelungskomplexen das strengere Recht gilt, jedoch entspricht dieses Ergebnis eher dem Ziel der InfoSoc-R1., ein hohes Schutzniveau für die Rechtsinhaber ${ }^{939}$ zu etablieren als die Schwerpunkttheorie, die zwangsläufig zu einer Verkürzung dieses Schutzes führt.

Da somit sowohl die Vorschriften der $\S \S 69$ a ff. UrhG als auch der $\S \S 95 a \mathrm{ff}$. UrhG parallel auf digitale Spiele Anwendung finden, unterliegen auch die technischen Maßnahmen, welche sowohl den Programmcode als auch die audiovisuellen Inhalte schützen, beiden Regelungskomplexen. In der Konsequenz setzt sich das strengere Regelungsregime durch mit der Folge, dass die Umgehung der supportlosen Online-DRMSysteme zum Zwecke der Wiederherstellung der Nutzungsmöglichkeit des digitalen Spiels gemäß § 95a Abs. 1 UrhG unzulässig ist.

Diese Ergebnisse können auch auf den sog. Jailbreak von Spielekonsolen übertragen werden. Wie in Kapitel 1 bereits erläutert worden ist, setzt das Ablaufenlassen von

\footnotetext{
937 Peukert, in Loewenheim, Handbuch des Urheberrechts, § 34, Rn. 8; Lambrecht, Bildschirmspiele, S. 241.

938 So auch BGH, GRUR 2013, 1035, 1037, Tz. 24; Hilgert, CR 2014, 354, 356; Roth, MMR 2013, 671,674 .

939 Siehe die InfoSoc-R1., Erwg. 4.
} 
wieder nutzbar gemachten digitalen Spielen auf Spielekonsolen einen sog. Jailbreak der Konsole voraus. ${ }^{940}$ Der Jailbreak erfordert eine Modifikation der Konsolen-Firmware mit dem Ziel, dass auch nicht autorisierte Spielkopien auf der Spielekonsole gespielt werden können. Die Authentifizierung der Spielkopie erfordert ein Zusammenspiel von Spielkopie und Spielekonsole, was der EuGH ausdrücklich als eine technische Maßnahme im Sinne des Art. 6 Abs. 3 der InfoSoc-RL angesehen hat. ${ }^{941}$

Die Firmware bzw. die von Unterprogrammen der Firmware durchgeführten Authentifizierungsmaßnahmen können daher als technische Maßnahmen i. S. d. § 95a Abs. 2 UrhG eingeordnet werden.

\section{e) Pflicht zur Zurverfügungstellung erforderlicher Mittel gemäß § 95b Abs. 1 UrhG}

Einen Ausweg aus dem Dilemma, wonach die Eliminierung der supportlosen Online-DRM-Systeme zwar nach § 69d Abs. 1 UrhG zulässig, nach § 95a Abs. 1 UrhG jedoch nicht erlaubt ist, könnte $\S 95 \mathrm{~b}$ Abs. 1 UrhG bieten.

Nach dieser Vorschrift sind Rechtsinhaber verpflichtet, den durch die in $\S 95 \mathrm{~b}$ Abs. 1 UrhG aufgelisteten Schrankenbestimmungen „Begünstigten, soweit sie rechtmäßig Zugang zum Werk oder Schutzgegenstand haben, die notwendigen Mittel zur Verfügung zu stellen, um von diesen Bestimmungen in dem erforderlichen Maße Gebrauch machen zu können“. ${ }^{942}$ Damit die Inhaber supportloser digitaler Spiele gegen den Rechtsinhaber einen Anspruch nach § 95b Abs. 2 UrhG geltend machen können, ist es erforderlich, dass sie auf "Mittel“ des Rechtsinhabers angewiesen sind, um von den in $\S 95 b$ Abs. 1 UrhG aufgelisteten Schrankenbestimmungen Gebrauch machen zu können. ${ }^{943}$

Welche „Mittel“ in diesem Fall zur Verfügung zu stellen sind, ist nicht vorgegeben, sodass ganz verschiedene Optionen in Betracht kommen. ${ }^{944}$ Daher ist es auch denkbar, dass der Rechtsinhaber mit Blick auf supportlose Online-DRM-Systeme beispielsweise ein Update für das digitale Spiel veröffentlicht, welches das Erfordernis der Authentifizierung beseitigt. ${ }^{945}$

Voraussetzung ist zunächst, dass die supportlosen Online-DRM-Systeme Nutzungshandlungen des Nutzers des digitalen Spiels blockieren, welche von einer in $\S 95 \mathrm{~b}$ Abs. 1 UrhG aufgelisteten Schrankenbestimmung gedeckt sind. Dies ist in Bezug auf die für das Ablaufenlassen des digitalen Spiels erforderliche Vervielfältigung der audiovisuellen Bestandteile in den Arbeitsspeicher bzw. auf die Festplatte des Nutzers

\footnotetext{
940 Siehe dazu oben, S. 24.

941 EuGH, GRUR 2014, 255, 257, Tz. 26.

$942 \S 95$ b Abs. 1 S. 1 UrhG; Dreier/Schulze/Specht, § 95b UrhG, Rn. 2.

943 Vgl. Fromm/Nordemann/Czychowski, § 95b UrhG, Rn. 16.

944 Dreier/Schulze/Specht, § 95b UrhG, Rn. 10.

945 Siehe dazu oben, S. 30 f.
} 
vorstellbar. Wie bereits dargestellt, handelt es sich hierbei jeweils um eine Vervielfältigung gemäß $\S 16$ Abs. 1 UrhG. ${ }^{946} \mathrm{Zu}$ einer solchen Verhinderung der Vervielfältigung audiovisueller Bestandteile in den Arbeitsspeicher kann es möglicherweise kommen, wenn eine servergebundene Authentifizierung bereits beim Start des Spiels erforderlich ist - wie z. B. beim Einloggen mit einem Spieleraccount -, der Support für diese Authentifizierung jedoch nicht mehr erbracht wird.

Im Folgenden wird untersucht, welche Schrankenbestimmungen die Vervielfältigung der audiovisuellen Bestandteile des digitalen Spiels gestatten und ob diese von $\S$ 95b Abs. 1 UrhG erfasst sind.

Mit Blick auf Privatperson, welche die Spielkopie nur zur Unterhaltung - und nicht etwa zu Forschungszwecken - nutzen, kommt als Rechtfertigung für eine solche Vervielfältigung die Schranke des $\S 53$ UrhG in Betracht. Gemäß § 95b Abs. 1 Nr. 6 UrhG setzen sich jedoch nur einige Bestimmungen dieser Schranke durch. Die digitale Vervielfältigung audiovisueller Werke zu privaten Zwecken ist nicht von $\S 95 \mathrm{~b}$ Abs. 1 UrhG erfasst. ${ }^{947}$

Eine weitere Schrankenbestimmung, auf welche sich der private Inhaber der Spielkopie berufen könnte, um einen Anspruch nach $\S 95$ b Abs. 2 UrhG gegen den Rechtsinhaber geltend zu machen, ist nicht ersichtlich.

Seit dem 1. März 2018 ist das „Gesetz zur Angleichung des Urheberrechts an die aktuellen Erfordernisse der Wissensgesellschaft“ (UrhWissG) in Kraft. Ziel dieser Novelle des UrhG ist es, bisherige Defizite in den gesetzlichen Schrankenbestimmungen zugunsten von Bildung und Wissenschaft zu beseitigen und die erlaubnisfreien Nutzungen für diesen Bereich im UrhG sowohl zu vereinfachen als auch zu erweitern. ${ }^{948}$ $\mathrm{Zu}$ diesem Zweck wurde mit den $\S \S 60 \mathrm{a} f f$. UrhG eine Reihe neuer Schrankenbestimmungen in das UrhG aufgenommen, welche spezifisch auf den Bereich Bildung und Wissenschaft zugeschnitten sind.

Der neue $\S 60 \mathrm{c}$ UrhG ersetzt sowohl $\S 52 \mathrm{a}$ Abs. $1 \mathrm{Nr} .2$ als auch $\S 53$ Abs. 2 Satz 1 Nr. 1 UrhG a. F. und übernimmt die in diesen Normen enthaltenen Regelungsgehalte. ${ }^{949}$ Abs. 1 dieser Vorschrift erlaubt die Vervielfältigung, Verbreitung und öffentliche Zugänglichmachung von bis zu $15 \%$ eines Werkes für einen bestimmt abgegrenzten Kreis von Personen für deren eigene wissenschaftliche Forschung sowie für einzelne Dritte, soweit dies der Überprüfung der Qualität wissenschaftlicher Forschung dient. Voraussetzung ist jedoch, dass die Forschung ,nicht kommerziell“" ist, worunter auch drittmittelfinanzierte Forschung an öffentlichen Hochschulen fallen kann. ${ }^{950}$ Bis zu $75 \%$ eines Werkes dürfen darüber hinaus gemäß § 60c Abs. 2 UrhG für die eigene wissenschaftliche Forschung genutzt werden.

\footnotetext{
946 Siehe dazu oben, S. $146 \mathrm{ff}$.

947 Vgl. Wandtke/Bullinger/Wandtke/Ohst, § 95b UrhG, Rn. 26.

948 BT-Drs. 18/12329, S. 2; siehe zum Ganzen auch de la Durantaye, GRUR 2017, 558.

949 BT-Drs. 18/12329, S. 39.

950 BT-Drs. 18/12329, S. 39.
} 
Damit ist die Vervielfältigung der audiovisuellen Inhalte des supportlosen digitalen Spiels auf der Festplatte oder in den Arbeitsspeicher - im Rahmen des jeweils anwendbaren prozentualen Nutzungsumfangs - zum Zwecke der Forschung von § 60c UrhG erlaubt. Zur Klarstellung sei an dieser Stelle noch einmal angeführt, dass sich die Erlaubnis nicht auch auf die Vervielfältigung der Steuerungssoftware in den Arbeitsspeicher oder auf der Festplatte erstreckt, wofür § 69d Abs. 1 UrhG eine Rechtfertigung enthält. Deshalb haben Forscher nach dem Ende des Supports - sofern die supportlosen Online-DRM-Systeme die Vervielfältigung verhindern - auch einen Anspruch gegen den Rechtsinhaber gemäß § 95b Abs. 1 Nr. 10 UrhG i. V. m. § 60c Abs. 1, 2 UrhG.

Die neu eingeführten $\S \S 60 \mathrm{e}, 60 \mathrm{f}$ UrhG enthalten Schrankenregelungen für öffentlich zugängliche Bibliotheken, die keine unmittelbaren oder mittelbaren kommerziellen Zwecke verfolgen, Archive, Einrichtungen im Bereich des Film- oder Tonerbes sowie für öffentlich zugängliche Museen und Bildungseinrichtungen (zusammen nachfolgend "Gedächtnisinstitutionen“).

$\S 60$ e Abs. 1 i. V. m. § 60f UrhG erlaubt es den genannten Gedächtnisinstitutionen, ein Werk aus ihrem Bestand bzw. ihrer Ausstellung unter anderem für Zwecke der Erhaltung und Restaurierung zu vervielfältigen oder vervielfältigen zu lassen. In eng umgrenzten Fällen dürfen die Gedächtnisinstitutionen die gemäß § 60e Abs. 1 UrhG hergestellten Vervielfältigungen darüber hinaus auch i. S. d. § 17 UrhG verbreiten.

$\S 60 \mathrm{e}$ Abs. 4 UrhG enthält die sog. Terminal-Schranke aus Art. 5 Abs. 3 der InfoSocRL, welche vor Inkrafttreten des UrhWissG in $\S 52 \mathrm{~b}$ UrhG geregelt war. ${ }^{951} \mathrm{Zu}$ beachten ist aber, dass nach dem neu gefassten $\S 95$ b Abs. 1 UrhG die Terminal-Schranke nicht durchsetzungsstark ist.

Es werden somit zwar Vervielfältigungen von den neuen Schrankenvorschriften der $\S \S 60 \mathrm{e}, \mathrm{f}$ UrhG erfasst. Allerdings sind diese Schrankenvorschriften im Gegensatz zu $\S 60 \mathrm{c}$ UrhG weitaus enger. So ist gemäß $\S 60 \mathrm{e}$ Abs. 1 UrhG die Vervielfältigung unter anderem nur zu Zwecken der Erhaltung und Restaurierung zulässig. Damit hatte der Gesetzgeber Maßnahmen zur digitalen Langzeitarchivierung im Sinne, bei denen eine Vervielfältigung eines Werkes auf einen neuen Träger und unter Umständen auch in einem neuen Format erforderlich sein kann. ${ }^{952}$ Diese zum Zwecke der Langzeitarchivierung notwendigen Vervielfältigungen bedürfen jedoch nicht zwingend auch einer Umgehung technischer Maßnahmen. Nur wenn eine Langzeitarchivierung durch die supportlosen Online-DRM-Systeme verhindert werden sollte, besteht auch ein Anspruch der Gedächtnisinstitutionen nach § 95b Abs. 2 UrhG. Das Gleiche gilt auch für das Verbreiten etwaiger restaurierter digitaler Spiele gemäß § 60e Abs. 2 und 3 UrhG.

Ein Anspruch gegen den Rechtsinhaber auf eine Zurverfügungstellung von Mitteln für die Umgehung technischer Maßnahmen, um von den in § 95b Abs. 2 UrhG aufgeführten Schrankenbestimmungen in dem erforderlichen Maße Gebrauch machen zu können, steht somit ausschließlich Forschern sowie Gedächtnisinstitutionen unter bestimmten Umständen zu.

\footnotetext{
951 BT-Drs. 18/12329, S. 43.

952 BT-Drs. 18/12329, S. 42.
} 


\section{Zulässigkeit weiterer Nutzungshandlungen}

Bisher ging es stets um die Zulässigkeit der Eliminierung von Online-DRM-Systemen selbst. Die Eliminierung kann jedoch mit urheberrechtlich relevanten Nutzungshandlungen einhergehen, welche wiederum selbst einer Rechtfertigung bedürfen.

Es ist nicht auszuschließen, dass im Einzelfall eine zumindest teilweise Rückübersetzung des maschinenlesbaren Programmcodes der Spielkopie in eine von Menschen lesbare Programmiersprache erforderlich ist, um herauszufinden, wie der Programmcode an welchen Stellen geändert werden muss, um die nicht spielbezogenen OnlineKomponenten zu eliminieren. ${ }^{953}$

Dieser auch als „Dekompilierung“954 bezeichnete Prozess greift in das Vervielfältigungsrecht gem. § 69c Nr. 1 UrhG und in das Umarbeitungsrecht gemäß $\S 69 \mathrm{c} \mathrm{Nr} .2$ UrhG des Rechtsinhabers ein. ${ }^{955}$

$\S 69 \mathrm{e}$ Abs. 1 UrhG erlaubt derartige Nutzungshandlungen, wenn sie unerlässlich sind, um die erforderlichen Informationen zur Herstellung der Interoperabilität eines unabhängig geschaffenen Computerprogramms mit anderen Programmen zu erhalten. Die Eliminierung der Online-DRM-Systeme dient aber nicht der Herstellung von Interoperabilität mit einem unabhängig geschaffenen Computerprogramm. Wie bereits erläutert, handelt es sich vielmehr um eine Fehlerberichtigung i. S. d. § 69d Abs. 1 UrhG. Aus diesem Grund kann die evtl. erforderliche Dekompilierung nicht durch $\S$ 69e Abs. 1 UrhG gerechtfertigt werden. ${ }^{956}$

Fraglich ist dann aber, ob die Dekompilierung vom Recht der Fehlerberichtigung selbst gemäß $§ 69 \mathrm{~d}$ Abs. 1 UrhG umfasst ist.

Dies wird zum Teil verneint. ${ }^{957}$ Begründet wird dies damit, dass $\S 69 \mathrm{e}$ UrhG eine abschließende Regelung über die Zulässigkeit der Dekompilierung treffe und damit eine Rückübersetzung nicht erlaubt sei, die zu anderen als den in dieser Vorschrift geregelten Zwecken vorgenommen wird.

Nach einer anderen Auffassung treffe $\S 69 \mathrm{e}$ UrhG mit Blick auf die urheberrechtliche Zulässigkeit der Dekompilierung von Computerprogrammen keine abschließende Regelung. ${ }^{958}$ Hierfür spreche die schlichte Notwendigkeit einer Dekompilierung, die

953 Nach Schweyer, Reverse Engineering, S. 107 ist die Fehlerberichtigung regelmäßig erst nach Rückübersetzung des Programms möglich.

954 Ausführlich dazu ebd., S. $81 \mathrm{ff}$.

955 Ebd., S. 87 f.

956 Dreier/Schulze/Dreier, § 69e UrhG, Rn. 12; Wandtke/Bullinger/Grützmacher, § 69e UrhG, Rn. 7; Ahlberg/Götting/Kaboth/Spies, § 69e UrhG, Rn. 7.

957 Schricker/Loewenheim/Spindler, § 69d UrhG, Rn. 3; Wandtke/Bullinger/Grützmacher, § 69d UrhG, Rn. 22; Dreier/Schulze/Dreier, § 69d UrhG, Rn. 10, 22; Lehmann, NJW 1993, 1822, 1824; Raubenheimer, CR 1996, 69, 76; Marly, JurPC 1992, 1652, 1662; Möhring/Nicolini/Kaboth/Spies, § 69d UrhG, Rn. 3.

958 Schweyer, Reverse Engineering, S. 107 ff.; Günther, CR 1994, 321, 327 f.; Hoeren/Schuhmacher, CR 2000, 137, 140; Lehmann, in Loewenheim, Handbuch des Urheberrechts, § 76, Rn. 30; Koch, NJW-CoR 1994, 293, 296; Vinje, CR 1993, 401, 404 f.; Dreyer/Kotthoff/Meckel/Hentsch/Kotthoff, § 69d UrhG, Rn. 7; nur bei Vorliegen eines schweren Programmfehlers: Fromm/Nordemann/Czychowski, § 69d UrhG, Rn. 20. 
zum Zwecke der Fehlerbeseitigung in Computerprogrammen sehr oft erforderlich sei. ${ }^{959}$ Darüber hinaus hätten $\S 69 \mathrm{~d}$ Abs. 1 UrhG und $\S 69$ e UrhG unterschiedliche Schutzrichtungen: $\S 69 \mathrm{~d}$ Abs. 1 UrhG schütze das Interesse des Programmanwenders an einem funktionierenden Programm, wohingegen $\S 69 \mathrm{e} \mathrm{UrhG} \mathrm{wettbewerbsbezogene}$ Interessen des Programmherstellers im Blick habe. ${ }^{960}$ Schließlich verweist $\S 69 \mathrm{~d}$ Abs. 1 UrhG auch ausdrücklich auf $\S 69 \mathrm{c}$ Nr. 2 UrhG und damit auf „Übersetzungen“ des Programms, worunter eben auch die Dekompilierung zu fassen sei. ${ }^{961}$ Dieser Ansicht ist zu folgen. Würde die Dekompilierung nicht von $\S 69 \mathrm{~d}$ Abs. 1 UrhG erfasst, würde das Recht des Nutzers zur Fehlerberichtigung unangemessen eingeschränkt werden.

Damit ist die Dekompilierung grundsätzlich auch im Rahmen der Fehlerberichtigung zulässig, jedoch nur, soweit sie erforderlich ist, etwa weil die mittels Dekompilation zu ermittelnden Informationen nicht anderweitig verfügbar sind. Es muss sich auf die Teile des Programms beschränkt werden, in denen der Programmcode des fehlerhaften Programmschutzes vermutet wird. ${ }^{962}$

Es ist bereits dargestellt worden, dass die Umgehung von supportlosen OnlineDRM-Systemen dauerhafte oder temporäre Veränderungen am Steuerungsprogramm des digitalen Spiels erforderlich machen kann. Hierbei kann es sich um eine Umarbeitung gemäß $\S 69 \mathrm{c}$ Nr. 2 UrhG handeln. Der Begriff umfasst alle Änderungen eines urheberrechtlich geschützten Computerprogramms. ${ }^{963}$ Die Veränderung des Programmcodes des Steuerungsprogramms zum Zwecke der Umgehung der supportlosen Online-DRM-Systeme ist als eine solche Umarbeitung einzuordnen.

Die Umarbeitung ist jedoch gemäß § 69d Abs. 1 UrhG zulässig. Dies ergibt sich aus den oben genannten Ausführungen, nach denen die Umgehung eines fehlerhaften Programmschutzmechanismus zum Zwecke der Fehlerberichtigung erlaubt sein kann. ${ }^{964}$ Dies gilt allerdings nur, soweit eine solche eigenhändige Fehlerberichtigung auch notwendig ist.

\section{Die Wiederherstellung des Online-Mehrspielermodus}

Im Folgenden wird die Rechtmäßigkeit der Wiederherstellung des Online-Mehrspielermodus und der dafür erforderlichen Maßnahmen untersucht.

\footnotetext{
959 Ausführlich dazu Schweyer, Reverse Engineering, S. 107 ff.

960 Vgl. Lehmann, in Loewenheim, Handbuch des Urheberrechts, § 76, Rn. 30.

961 Hoeren/Schuhmacher, CR 2000, 137, 140.

962 Vgl. Schweyer, Reverse Engineering, S. 112.

963 Dreier/Schulze/Dreier, § 69c UrhG, Rn. 15.

964 Siehe dazu oben, S. $160 \mathrm{f}$.
} 
Zunächst wird die Frage beleuchtet, ob es zulässig ist, die Spielkopie ohne Erlaubnis des Publishers mit einer anderen Spielkopie (Tunneling) oder mit einem neuen Masterserver zu verbinden (I.). Im Anschluss daran soll das in Kapitel 1 näher erläuterte Reverse Engineering von Kommunikationsprotokoll und Payload ${ }^{965}$ auf seine Rechtmäßigkeit hin untersucht werden (II.).

Schließlich wird geprüft, ob der sich an die Wiederherstellung der Nutzungsmöglichkeit anschließende Betrieb des inoffiziellen Masterservers sowie damit zusammenhängende Maßnahmen zulässig sind (III.).

\section{Die Herstellung einer unerlaubten Verbindung}

In Kapitel 1 dieser Arbeit sind verschiedene Möglichkeiten zur Wiederherstellung des Online-Mehrspielermodus dargestellt worden. ${ }^{966}$

Falls das Spiel einen LAN-Mehrspielermodus enthält, kann mithilfe eines auf dem Endgerät des Spielers zu installierenden Programms eine Verbindung über das Internet mit einem anderen Spieler hergestellt werden. Das Programm täuscht der Spielkopie dabei vor, dass es innerhalb eines LAN mit einem anderen Spieler verbunden ist. Hierfür ist grundsätzlich weder eine Verbindung mit einem (neuen) Server noch eine Modifikation der Spielkopie erforderlich.

Falls das Spiel keinen LAN-Mehrspielermodus enthält, ist die Wiederherstellung des Online-Mehrspielermodus nur durch die Einrichtung einer Client-Server-Verbindung zwischen der Spielkopie und einem neuen Masterserver möglich. Hierfür kommen zwei Wege in Betracht: Zum einen kann der Inhaber der Spielkopie einen sog. custom DNS-Server einrichten, welcher die von der Spielkopie angefragte IP-Adresse des ursprünglichen Masterservers mit der IP-Adresse eines neuen Servers verbindet. Alternativ kann die URL des alten Servers innerhalb der Spielkopie mit der URL des neuen Servers temporär oder dauerhaft überschrieben werden. ${ }^{967}$

Nutzungsbedingungen der Publisher, welchen der Erwerber des Spiels zustimmen muss, untersagen derartige Verbindungen jedoch zum Teil ausdrücklich. So verbietet beispielsweise Ziff. 1. C. ix der Endnutzerlizenzvereinbarung des Publishers Blizzard vom 11. September 2018 jegliche Form von

„Förderung, Aufbau oder Aufrechterhaltung einer nicht genehmigten Verbindung zur Plattform ${ }^{968}$, einschließlich, jedoch nicht beschränkt auf (i) Verbindungen zu nicht genehmigten Servern, die die Plattform emulieren oder dies versuchen [...] “969

965 Siehe dazu oben, S. 28 ff.

966 Ausführlich dazu oben, S. 26 ff.

967 Siehe zum Ganzen oben, S. 26 ff.

968 Der Begriff „Plattform“ meint ausweislich der Blizzard-Endnutzerlizenzvereinbarung unter anderem die von Blizzard verlegten digitalen Spiele.

969 Die Blizzard-Endnutzerlizenzvereinbarung ist abrufbar unter http://eu.blizzard.com/dede/company/legal/eula. 
Bei der soeben zitierten Bestimmung handelt es sich um eine vorformulierte Klausel gemäß $§ 305$ Abs. 1 Satz 1 BGB. Die Auslegung dieser Klausel ergibt, dass vom Publisher nicht genehmigte Verbindungen der Spielkopie beispielsweise mit einem inoffiziellen Masterserver unzulässig sind. Dabei ist die Konnektierung der Spielkopie mit nicht genehmigten Servern nur als ein Beispiel in einem nicht abschließenden Katalog aufgeführt, sodass die Klausel durchaus dahingehend ausgelegt werden kann, dass auch andere Maßnahmen wie das Tunneling nicht gestattet sind. Fraglich ist jedoch, ob diese Einschränkungen der Nutzungsmöglichkeit der Spielkopie wirksam sind.

Für die Prüfung der Wirksamkeit solcher Klauseln ist danach zu unterscheiden, ob die Herstellung der unerlaubten Verbindung eine Veränderung des Programmcodes der Spielkopie erfordert oder nicht.

\section{Herstellung der Verbindung durch Veränderung der Spielkopie}

$\S 69 \mathrm{~d}$ Abs. 1 UrhG erlaubt dem Anwender, Vervielfältigungen und Umarbeitungen des Computerprogramms im Sinne von $\S 69 \mathrm{c} \mathrm{Nr}$. 1 und 2 UrhG vorzunehmen, wenn sie für eine bestimmungsgemäße Benutzung einschließlich der Fehlerberichtigung notwendig sind. Die Änderung der Programmkopie des digitalen Spiels dahingehend, dass sie sich mit einem neuen Masterserver verbindet, kann als eine Umarbeitung angesehen werden.

$\S 69 \mathrm{~d}$ Abs. 1 UrhG enthält dabei einen zwingenden Kern, von dem durch vertragliche Vereinbarungen nicht abgewichen werden darf. ${ }^{970} \mathrm{Zu}$ dem zwingenden Kern gehört unter anderem die Fehlerberichtigung. ${ }^{971}$ AGB, welche gegen diesen zwingenden Kern verstoßen, sind gem. $§ 307$ Abs. 2 Nr. 1 BGB unwirksam. ${ }^{972}$

Es ist im Zusammenhang mit der Eliminierung von supportlosen Online-DRM-Systemen bereits erläutert worden, dass die dauerhafte Unterbrechung der Client-ServerKommunikation einen Fehler gemäß $§ 69 \mathrm{~d}$ Abs. 1 UrhG darstellt, welcher es dem Nutzer des digitalen Spiels - soweit erforderlich - erlaubt, diesen Fehler durch Veränderungen am Programm zu berichtigen. ${ }^{973}$

In gleicher Weise ist die dauerhafte Unterbrechung der für den Support des OnlineMehrspielermodus erforderlichen Client-Server-Kommunikation als Fehler i. S. d. § 69d Abs. 1 UrhG einzuordnen. Durch die Supportbeendigung kann das Spiel keine Verbindung mehr mit den Masterservern aufbauen und damit keine - an und für sich noch vorhandene - Spieleserver auflisten, welche der Spieler für ein MehrspielerMatch nutzen könnte. Zwar gestattet das Recht zur Fehlerberichtigung nicht, die Software weiterzuentwickeln oder zu verbessern. ${ }^{974}$ Bei der Veränderung der Spielkopie

\footnotetext{
970 Siehe dazu oben, S. $160 \mathrm{f}$.

971 Dreier/Schulze/Dreier, § 69d UrhG, Rn. 12; Redeker, IT-Recht, Rn. 65; Schweyer, Reverse Engineering, S. 116.

972 Vgl. Wandtke/Bullinger/Grützmacher, § 69d UrhG, Rn. 41.

973 Siehe dazu oben, S. $166 \mathrm{f}$.

974 Schricker/Loewenheim/Spindler, § 69d UrhG, Rn. 10.
} 
zum Zweck der Verbindung mit einem neuen Masterserver handelt es sich jedoch lediglich um eine Erhaltung der Nutzungsmöglichkeit, welche als solche vom Fehlerberichtigungsrecht umfasst ist. ${ }^{975}$

Doch auch dieses Recht des Spielers steht unter dem Vorbehalt der Erforderlichkeit. ${ }^{976}$ Solange und soweit der Supportverpflichtete die Client-Server-Kommunikation mit seinen eigenen Masterservern aufrechterhält, ist eine eigenhändige Fehlerberichtigung des Programmanwenders nicht erforderlich und damit ausgeschlossen. $\mathrm{Zu}-$ lässig ist die Beseitigung der Funktionsstörung damit erst ab dem Zeitpunkt, ab dem der Publisher keinen Support mehr erbringt.

Da die zitierte Klausel des Publishers Blizzard nicht zwischen dem Zeitraum vor und nach dem Ende des Supports unterscheidet und deshalb eine Fehlerberichtigung auch nach dem Supportende untersagt, schränkt sie unzulässigerweise den nicht abdingbaren Kern an Nutzungshandlungen des Erwerbers ein, zu dem auch die Fehlerberichtigung gehört. Damit verstößt sie gegen $\S 307$ Abs. 2 Nr. 1 BGB und ist unwirksam.

\section{Herstellung der Verbindung ohne Veränderung der Spielkopie}

Wie bereits dargelegt worden ist, bedarf die Herstellung einer vom Publisher nicht genehmigten Verbindung nicht stets einer Umarbeitung der Software der Spielkopie. So erfordert die Wiederherstellung des Online-Mehrspielermodus im Wege des Tunnelings oder mithilfe eines custom-DNS-Servers keine Veränderungen des Programmcodes selbst. ${ }^{977}$ Mit Blick auf beide Verbindungsmöglichkeiten ist der Prüfungsmaßstab für die Zulässigkeit der zitierten Klausel mangels urheberrechtlich relevanter Umarbeitungen des Programmcodes daher nicht $\S 69 \mathrm{~d}$ Abs. 1 UrhG, sondern ausschließlich $\S 307$ Abs. 1 Satz 1 BGB.

Die oben zitierte Klausel des Publishers Blizzard verbietet alle vom Publisher nicht genehmigten Verbindungen, wodurch der Support von Online-Komponenten des digitalen Spiels wiederhergestellt werden könnte. Der Publisher erlaubt damit eine Nutzung der Spielkopie nur mit einer von ihm genehmigten Client-Server-Verbindung. Die Klausel ist daher mit sog. CPU-Klauseln vergleichbar, die Regelungen in Software-Überlassungsverträgen darstellen, welche die erlaubte Nutzung eines Computerprogramms nur auf einen bestimmten Rechner beschränken. ${ }^{978}$ So wie CPU-Klauseln dem Nutzer vorschreiben, die Software nur auf einem bestimmten Rechner zu benutzen, schreibt die oben zitierte AGB des Publishers Blizzard dem Erwerber des digitalen Spiels vor, das Spiel nur mit einer vom Publisher genehmigten Client-Server-Verbindung zu verwenden.

\footnotetext{
975 So auch für die Portierung Wandtke/Bullinger/Grützmacher, § 69d UrhG, Rn. 21; Dreier/Schulze/Dreier, $\S 69 \mathrm{~d}$ UrhG, Rn. 8.

976 Vgl. Dreier/Schulze/Dreier, § 69d UrhG, Rn. 11.

977 Siehe dazu oben, S. 26 ff.

978 Spindler/Schuster/Wiebe, § 69d UrhG, Rn. 34.
} 
Die herrschende Lehre vertritt die Auffassung, dass CPU-Klauseln, die in kaufvertragliche Softwareüberlassungsverträge einbezogen wurden, unwirksam sind. ${ }^{979}$

Zwar sei das Interesse des Softwareherstellers an einer wirtschaftlichen und sachgerechten Verwertung der Software anzuerkennen, was eine Nutzung des Computerprogramms auf mehr als einem Gerät ohne Erlaubnis des Softwareherstellers ausschließe. ${ }^{980}$ Die CPU-Klauseln gingen jedoch über eine rein quantitative Beschränkung der Nutzungsmöglichkeit hinaus, da der Käufer der Software ihretwegen mit der Kaufsache nicht im Rahmen des technisch Möglichen frei verfahren könne. ${ }^{981}$ So würde er durch CPU-Klauseln beispielsweise an einem notwendigen Hardware-Wechsel wegen eines Defekts des ursprünglichen Rechners gehindert. ${ }^{982}$ Das Interesse des Softwareherstellers, an einer Verbreitung seiner Software wirtschaftlich zu partizipieren, könne eine solch weitgehende qualitative Nutzungseinschränkung nicht rechtfertigen. ${ }^{983}$

Maßgeblich für eine Interessenabwägung ist damit einerseits das Interesse des Nutzers, mit der Software im Rahmen des technisch Möglichen frei verfahren zu können, und andererseits das Interesse des Softewareherstellers, an bestimmten Nutzungen der Software durch ihren Erwerber - wie eben ihrer Verbreitung oder der Installation auf einem anderen Rechner - wirtschaftlich zu partizipieren.

Zwar ist das Interesse des Supportverpflichteten, an der Nutzung des Online-Mehrspielermodus während des Supportzeitraums wirtschaftlich zu partizipieren, grundsätzlich höher zu gewichten als das Interesse des Erwerbers des digitalen Spiels, ebendieses unter Verwendung einer anderen Tunneling- oder Client-Server-Verbindung zu nutzen. Nach dem Ende des Supports kehrt sich dieses Verhältnis jedoch um. Angesichts schwindender Spielerzahlen ist es für den Supportverpflichteten nicht mehr wirtschaftlich, den Support des Online-Mehrspielermodus aufrechtzuerhalten. ${ }^{984} \mathrm{Da}-$ mit hat der Supportverpflichtete auch kein wirtschaftliches Partizipationsinteresse mehr an der Nutzung des Online-Mehrspielermodus durch den Erwerber des digitalen Spiels, denn anderenfalls hätte er den Support nicht beendet. Wie bereits dargestellt, sind die Erwerber von Inklusiv-, Konsolen- und Free-to-Play-Spielen auch noch nach

979 Dreier/Schulze/Dreier, § 69c UrhG, Rn. 33; Schricker/Loewenheim/Spindler, § 69d UrhG, Rn. 15; Wandtke/Bullinger/Grützmacher, § 69c UrhG, Rn. 42; Dreyer/Kotthoff/Meckel/Hentsch/Kotthoff, § 69d UrhG, Rn. 13; Spindler/Schuster/Wiebe, § 69d UrhG, Rn. 34; Haberstumpf, in Lehmann, Rechtsschutz und Verwertung von Computerprogrammen, 1. Teil, II., Rn. 165; Czychowski/Siesmayer, in Kilian/Heussen, Computerrechtshandbuch, Abschnitt 1, Teil 2, 20.4, Rn. 137; so auch OLG Frankfurt a. M., NJW-RR 1995, 182, 182.

980 Haberstumpf, in Lehmann, Rechtsschutz und Verwertung von Computerprogrammen, 1. Teil, II., Rn. 165.

981 Schricker/Loewenheim/Spindler, § 69d UrhG, Rn. 15.

982 Haberstumpf, in Lehmann, Rechtsschutz und Verwertung von Computerprogrammen, 1. Teil, II., Rn. 165; Dreyer/Kotthoff/Meckel/Hentsch/Kotthoff, § 69d UrhG, Rn. 13.

983 Vgl. Haberstumpf, in Lehmann, Rechtsschutz und Verwertung von Computerprogrammen, 1. Teil, II., Rn. 165; Dreyer/Kotthoff/Meckel/Hentsch/Kotthoff, § 69d UrhG, Rn. 13.

984 Siehe dazu die Einleitung. 
dem Ende des Supports dazu berechtigt, den Online-Mehrspielermodus zu benutzen. ${ }^{985}$ Durch vorformulierte Klauseln, welche den Erwerbern die Herstellung von mit dem „offiziellen“ Support des Publishers vergleichbaren Verbindungen pauschal untersagen, wird den Nutzern jedoch jede Möglichkeit genommen, vom Online-Mehrspielermodus auch noch nach der Beendigung des „offiziellen“ Supports Gebrauch machen zu können, wodurch ihre Nutzungsinteressen unangemessen beeinträchtigt werden.

Damit sind Klauseln wie die oben zitierte, welche die Herstellung von nicht genehmigten Verbindungen per se untersagen - also ohne danach zu differenzieren, ob diese Verbindung vor oder nach dem Ende des Supports erfolgt - gemäß § 307 Abs. 1 Satz 1 BGB unwirksam.

Im Ergebnis ist damit festzuhalten, dass sowohl die Verbindung der Spielkopie mit einem privaten - vom Publisher nicht genehmigten - Server als auch die Herstellung einer Verbindung mit einer anderen Spielkopie im Wege des Tunnelings nach dem Ende des Supports erlaubt ist und dass vorformulierte Klauseln, welche dies pauschal verbieten, unzulässig sind.

\section{Das Reverse Engineering des Kommunikationsprotokolls}

Wenngleich der Aufbau einer Verbindung mit einem ,inoffiziellen“ Masterserver nach dem Ende des Supports des Online-Mehrspielermodus zulässig ist, kann eine Kommunikation zwischen Server und Spielkopie nur dann erfolgen, wenn sie protokollgemäß vonstattengeht, wenn also die ausgetauschten Nachrichten zwischen Server und Clientprogramm beispielsweise ein bestimmes Format haben sowie auf eine festgelegte Art und Weise anfangen und enden.

Wie bereits in Kapitel 1 dieser Arbeit dargestellt, sind Informationen über Protokolle, die von Publishern für die Kommunikation zwischen Masterserver und Spielclient eingesetzt werden, nicht immer frei verfügbar. ${ }^{986}$

Ziel des Reverse Engineerings des Kommunikationsprotokolls ist es daher, die Informationen herauszufinden, die notwendig sind, um eine protokollgemäße ClientServer-Kommunikation programmiertechnisch umsetzen zu können.

Es stellt sich dabei aber die Frage, ob im Zuge des Reverse Engineerings Rechte des Publishers an dem Kommunikationsprotokoll verletzt werden.

\section{Verletzung eines nach dem UrhG geschützten Rechts am Kommuni- kationsprotokoll}

In Betracht kommt zunächst die Verletzung eines nach dem UrhG geschützten Rechts am Kommunikationsprotokoll. Hierfür ist erforderlich, dass das Kommunikationsprotokoll ein Werk darstellt (a)) oder auf andere Weise nach dem UrhG geschützt ist (b)).

\footnotetext{
985 Siehe dazu oben, S. 149 ff.

986 Siehe dazu oben, S. $28 \mathrm{ff}$.
} 


\section{a) Schutz als Werk gemäß § 69a Abs. 1, 2 UrhG}

Ausgangspunkt ist hierbei $\S 69 \mathrm{a}$ Abs. 2 UrhG, welcher festlegt, dass der urheberrechtliche Schutz für alle Ausdrucksformen eines Computerprogramms gilt. § 69a Abs. 2 Satz 2 UrhG erstreckt diesen Schutz auch auf Schnittstellen, worunter Elemente eines Computerprogramms verstanden werden, welche zur Interaktion mit externen Computerprogrammen bestimmt oder geeignet sind. ${ }^{987}$

$\S 69 \mathrm{a}$ Abs. 2 UrhG schließt jedoch aus seinem Schutzbereich die Ideen und Grundsätze aus, die einem Element des Computerprogramms einschließlich seiner Schnittstellen zugrunde liegen. Die Literatur ordnet Kommunikationsprotokolle als Grundsätze bzw. Ideen in diesem Sinne ein und versagt ihnen daher den Schutz nach dem UrhG. ${ }^{988}$ Dieser Ansicht ist zu folgen. Die Kommunikationsprotokolle legen fest, auf welche Art und Weise eine Kommunikation zwischen zwei Computerprogrammen, die über eine Schnittstelle miteinander verbunden sind, abzulaufen hat. Die Kommunikationsprotokolle geben damit eine bestimmte Funktionalität des Computerprogramms vor. Funktionalitäten sind jedoch keine Ausdrucksformen von Computerprogrammen und damit auch nicht nach $\S 69 \mathrm{a}$ Abs. 2 UrhG schutzfähig. ${ }^{989}$

Es ist zwar denkbar, dass die programmiertechnische Umsetzung dieser Funktionalität, die grundsätzlich im Ermessen des Programmierers liegt ${ }^{990}$, Urheberrechtsschutz genießen kann. Es ist jedoch Ziel der Computerprogramm-Rl., die Interoperabilität zwischen Computerprogrammen möglichst weitgehend sicherzustellen, weshalb der Urheberrechtsschutz von Schnittstellen oder eng mit diesen zusammenhängenden Komponenten nur sehr zurückhaltend bejaht werden sollte. ${ }^{991}$ Dementsprechend sind die Anforderungen an einen Urheberrechtsschutz an die programmiertechnische Umsetzung der Protokoll-Vorgaben entsprechend hoch. Doch selbst wenn - einen Urheberrechtsschutz unterstellt - die programmiertechnische Umsetzung der ProtokollVorgaben durch den Anwender mit der Programmierung des Publishers bzw. Entwicklers identisch sein sollte, wird es sich hierbei in der Regel um eine unabhängige Doppelschöpfung handeln, die als solche keine Verletzung des Urheberrechts darstellt ${ }^{992}$. Denn der Anwender, welcher die Protokollvorgaben herausfinden und für seinen eigenen Masterserver implementieren möchte, hat in der Regel keinen Zugriff auf die Server-Software des Publishers bzw. Entwicklers - erst recht dann nicht, wenn der offizielle Masterserver gar nicht mehr betrieben wird.

987 Vgl. Dreier/Schulze/Dreier, § 69a UrhG, Rn. 23.

988 So Koch, GRUR 1997, 417, 422; Pilny, GRUR Int. 1990, 431, 440; so auch ohne Begründung Ensthaler/Bock, GRUR 2009, 1, 6.

989 So in Bezug auf Art. 1 Abs. 2 der RL 91/250 EWG der EuGH, EuZW 2012, 584, 586, Tz. 46 SAS Institute.

990 So Schweyer, Reverse Engineering, S. 64.

991 Lehmann, in Lehmann, Rechtsschutz und Verwertung von Computerprogrammen, Einleitung I.A, Rn. 7.

992 Vgl. dazu Dreier/Schulze/Dreier/Specht, § 97 UrhG, Rn. 11. 
Aus diesen Gründen ist ein Urheberrechtsschutz des Kommunikationsprotokolls und in der Regel auch eine urheberrechtsrelevante Verletzung der - eventuell im Einzelfall schutzfähigen - programmiertechnischen Umsetzung desselben nicht gegeben.

\section{b) Schutz als technische Maßnahme oder Programmschutzmechanismus}

In Betracht kommt weiterhin ein Schutz des Kommunikationsprotokolls als technische Maßnahme gemäß $\S 95 \mathrm{a}$ Abs. 2 UrhG bzw. als Programmschutzmechanismus gemäß § 69f Abs. 2 UrhG ${ }^{993}$

Im Sixth Triennial Section 1201 Proceeding wurde die Ansicht vertreten, dass Server-Protokolle insbesondere dann, wenn die Kommunikation zwischen Client und Server verschlüsselt erfolgt, den Zugang zu einem urheberrechtlich geschützten Werk kontrollieren und damit als ,technological measure“ im Sinne des sec. 1201(a)(1)(A) des amerikanischen Copyright Act einzuordnen seien. ${ }^{994}$

Diese Auffassung ist auf das deutsche und europäische Recht allerdings nicht übertragbar.

Voraussetzung für einen Schutz nach § 95a Abs. 2 UrhG ist, dass die Maßnahme „im normalen Betrieb“ dazu bestimmt ist, Handlungen, die nach dem UrhG geschützte Werke und Leistungen betreffen, zu verhindern oder einzuschränken. ${ }^{995}$ Diese Voraussetzung ist jedoch bei Kommunikationsprotokollen nicht gegeben. Ihr Zweck besteht in der Etablierung von Standards für einen reibungslosen Ablauf einer Client-ServerKommunikation. Nun mag die Unkenntnis von den Protokoll-Spezifikationen und eine etwaige Verschlüsselung der ausgetauschten Datenpakete die Wiederherstellung der Nutzungsmöglichkeit der spielbezogenen Online-Komponenten im Einzelfall erschweren oder faktisch sogar ausschließen. Dabei handelt es sich aber nur um einen Nebeneffekt, der für eine Einordnung des Protokolls als technische Maßnahme gemäß § 95a Abs. 2 UrhG nicht ausreicht.

Das Gleiche gilt für Programmschutzmechanismen gemäß § 69f Abs. 2 UrhG, welche dazu bestimmt sein müssen, Urheberrechtsverletzungen in Bezug auf Computerprogramme zu verhindern. ${ }^{996}$

Damit scheidet ein Schutz des Kommunikationsprotokolls als technische Maßnahme bzw. als Programmschutzmechanismus aus.

993 Siehe zu den Definitionen und Tatbestandsmerkmalen beider Begriffe oben, S. $152 \mathrm{ff}$. sowie S. $157 \mathrm{ff}$.

994 Vgl. Electronic Frontier Foundation, Comments of the Electronic Frontier Foundation (2015), S. 4, abrufbar unter http://copyright.gov/1201/2015/comments020615/InitialComments_longform_EFF_Class23.pdf.

995 Siehe dazu oben, S. $152 \mathrm{ff}$.

996 Vgl. Schricker/Loewenheim/Spindler, § 69f UrhG, Rn. 10; Möhring/Nicolini/Kaboth/Spies, § 69f UrhG, Rn. 8; Wand, Technische Schutzmaßnahmen, S. 146. 


\section{Verletzung eines Patents am Kommunikationsprotokoll}

Neben der Verletzung von urheberrechtlichen Vorschriften durch das Reverse Engineering kommt auch die Verletzung eines Patents in Betracht, welches für das Kommunikationsprotokoll besteht. Der Patentschutz setzt nach $\S 1$ Abs. 1 PatG voraus, dass es sich bei dem zu schützenden Gegenstand um eine Erfindung auf technischem Gebiet handelt, die neu ist, auf einer erfinderischen Tätigkeit beruht und gewerblich anwendbar ist. ${ }^{997}$

Vom Begriff der Erfindung ausgeschlossen sind gemäß $§ 1$ Abs. 3 Nr. 3, Abs. 4 PatG allerdings u. a. Programme für Datenverarbeitungsanlagen, sofern für sie als solche Schutz begehrt wird. Das bedeutet jedoch nicht, dass Computerprogramme schlechthin vom Patentschutz ausgeschlossen wären, was auch nicht mit Art. 27 Abs. 1 Satz 1 des Übereinkommens über handelsbezogene Aspekte der Rechte des geistigen Eigentums (im Folgenden „TRIPS-Abkommen“) in Einklang zu bringen wäre, wonach grundsätzlich „Patente für Erfindungen auf allen Gebieten der Technik erhältlich“ sein müssen. ${ }^{998}$ Für Computerprogramme bzw. computerimplementierte Erfindungen bedeutet dies, dass sie Patentschutz erlangen können, soweit sie ein über das Computerprogramm hinausgehendes technisches Problem lösen. ${ }^{999}$ Vor diesem Hintergrund können auch Kommunikationsprotokolle durchaus Patentschutz genießen. ${ }^{1000}$ Die Art und Weise der Kommunikation von Daten kann nämlich ein technisches Problem darstellen, dessen Lösung die Anforderungen an eine Erfindung gemäß $§ 1$ Abs. 1 PatG durchaus erfüllen kann. ${ }^{1001}$

Unter Voraussetzung eines bestehenden Patentschutzes für das Kommunikationsprotokoll ist jedoch fraglich, ob die Implementierung des geschützten Kommunikationsprotokolls ohne Einholung einer Lizenz vom Rechtsinhaber durch den Anwender eine Verletzung des Patents darstellt.

$\S 9$ PatG gewährt dem Patentinhaber ein Ausschließlichkeitsrecht. ${ }^{1002}$ Je nachdem, was für eine Patentkategorie gewählt worden ist, darf der Patentinhaber es Dritten untersagen, ein Erzeugnis, das Gegenstand des Patents ist, u. a. herzustellen, in Verkehr zu bringen oder zu gebrauchen oder - falls Gegenstand des Patents ein Verfahren ist das geschützte Verfahren anzuwenden. ${ }^{1003}$ Verfahrenspatente schützen ein bestimmtes erfinderisch-technisches Verfahren. ${ }^{1004}$

\footnotetext{
997 Vgl. Osterrieth, Patentrecht, Rn. 332, 504 ff.

998 Ebd., Rn. 375; vgl. auch Mes/Mes, § 1 PatG, Rn. 122.

999 BGH, GRUR 2009, 479, 480 - Steuerungseinrichtung für Untersuchungsmodalitäten; Mes/Mes, § 1 PatG, Rn. 122.

${ }^{1000}$ Schweyer, Reverse Engineering, S. 64, 302; Pilny, GRUR Int. 1990, 431, 441.

1001 Schweyer, Reverse Engineering, S. 302.

1002 Benkard/Scharen, § 9 PatG, Rn. 4.

$1003 \S 9$ S. 2 PatG.

1004 Osterrieth, Patentrecht, Rn. 366.
} 
Für ein Kommunikationsprotokoll kommt die Einordnung als Verfahrenspatent in Betracht. ${ }^{1005}$ Die Anwendung eines Verfahrenspatents i. S. d. § 9 Satz 2 Nr. 2 PatG meint den bestimmungsgemäßen Gebrauch dieses Verfahrens. ${ }^{1006}$ Ein solcher Gebrauch kann im Einzelfall gegeben sein, wenn der Anwender das einmal ermittelte Kommunikationsprotokoll für den Betrieb des neuen Masterservers implementiert hat.

$\mathrm{Zu}$ beachten ist allerdings, dass $\S 11 \mathrm{PatG}$ bestimmte Handlungen in Bezug auf den vom Patent geschützten Gegenstand erlaubt. Dazu zählen gem. § 11 Nr. 1 PatG Handlungen, die im privaten Bereich und zu nichtgewerblichen Zwecken vorgenommen werden.

Der private Bereich eines Menschen umfasst seine Privatsphäre wie seine Familie, seinen Haushalt und Aktivitäten, denen er zur Unterhaltung nachgeht. ${ }^{1007}$ Die Handlungen erfolgen darüber hinaus zu nichtgewerblichen Zwecken, wenn der Handelnde mit ihnen keinen Erwerbszweck verfolgt. ${ }^{1008}$

Sobald der Anwender - was im Regelfall anzunehmen sein dürfte - das patentrechtlich geschützte Protokoll lediglich in seiner Privatsphäre und ohne Verfolgung von Erwerbszwecken zur Wiederherstellung des Online-Mehrspielermodus implementiert, ist eine Verletzung eines etwaigen Patents an dem Kommunikationsprotokoll zu verneinen.

Anders ist die Lage allerdings mit Blick auf Gedächtnisinstitutionen wie Bibliotheken und Museen sowie auf Forscher zu beurteilen. Da die Tatbestandsmerkmale - privater Bereich und nichtgewerblicher Zweck - kumulativ vorliegen müssen ${ }^{1009}$, können sich Gedächtnisinstitutionen nicht auf $\S 11$ Nr. 1 PatG berufen, weil die Handlungen hier schon nicht „im privaten Bereich“ im Sinne dieser Vorschrift stattfinden. Das Gleiche gilt für Forscher, sofern sie mit ihrer Forschung einen Erwerbszweck verfolgen, der ebenfalls die Nichtgewerblichkeit ausschließ $\mathrm{t}^{1010}$.

\section{Verletzung von Vorschriften aus dem StGB}

Das Reverse Engineering des Kommunikationsprotokolls ist mit einem Abfangen sowie einer Analyse von Datenpaketen verbunden, die von der Spielkopie ausgesandt oder - falls der offizielle Masterserver des Supportverpflichteten noch online ist zwischen Server und Spielkopie ausgetauscht werden. ${ }^{1011}$ Dieses Abfangen und Analysieren der Datenpakete könnte gemäß §§ 202a, 202b sowie 303a StGB strafbar sein.

\footnotetext{
1005 Siehe z. B. das Patent des Patentinhabers Qualcomm Inc., Az. 60034551.3 für ein Kommunikationsprotokoll zwischen einer Kommunikationseinrichtung und einem externen Zusatzgerät, welches als ein Verfahrenspatent angemeldet worden ist, abrufbar unter https://register.dpma.de/DPMAregister/pat/PatSchrifteneinsicht?docId=DE000060034551T2\&p age $=3 \&$ dpi $=150 \&$ lang $=$ de .

1006 Mes/Mes, § 9 PatG, Rn. 56; Benkard/Scharen, § 9 PatG, Rn. 49.

1007 Vgl. Mes/Mes, § 11 PatG, Rn. 3; Benkard/Scharen, § 11 PatG, Rn. 3.

1008 Ann, in Kraßer/Ann, Patentrecht, § 33, Rn. 239.

1009 Benkard/Scharen, § 11 PatG, Rn. 3.

1010 Vgl. Ann, in Kraßer/Ann, Patentrecht, § 33, Rn. 239.

1011 Siehe dazu oben, S. $28 \mathrm{ff}$.
} 


\section{a) Strafbarkeit gemäß § 202a Abs. 1 StGB}

Die Vorschrift bestraft den Täter, welcher sich oder einem anderen unter Überwindung einer Zugangssicherung unbefugt Zugang zu Daten verschafft.

$\S 202$ a Abs. 2 StGB legt dabei fest, dass die Daten ,elektronisch, magnetisch oder sonst nicht unmittelbar wahrnehmbar gespeichert [sein] oder übermittelt werden" müssen. Die Vorschrift definiert den Begriff „Daten“ nicht. Er ist jedoch grundsätzlich weit zu verstehen, sodass hierunter die Darstellung aller Informationen mithilfe bestimmter Codes fällt. ${ }^{1012}$ Die Daten sind nicht unmittelbar wahrnehmbar, wenn sie erst nach einer technischen Umwandlung sinnlich wahrgenommen werden können. ${ }^{1013}$ Die Übermittlung meint die Weitergabe der Daten von der einen zu einer anderen Speicherungsstelle. ${ }^{1014}$ Die Daten müssen darüber hinaus nicht für den Täter bestimmt und gegen unberechtigten Zugang besonders gesichert sein. Ob die Daten für den Täter bestimmt sind, hängt davon ab, ob sie ,nach dem Willen desjenigen, der zum Zeitpunkt der Tat die Verfügungsmacht über die Daten innehat", dem Täter zur Verfügung stehen sollen. ${ }^{1015}$ Die Verfügungsmacht wird durch das Abspeichern der Daten oder aber im Falle der Übermittlung mit ihrem Empfang begründet. ${ }^{1016}$ Darüber hinaus ist auch die übermittelnde Stelle Berechtigter im Sinne des $\S 202 \mathrm{a}$ Abs. 1 StGB. ${ }^{1017}$

Eine Zugangssicherung liegt vor, wenn Maßnahmen getroffen wurden, welche den Zugriff auf die Daten verhindern oder zumindest nicht unerheblich erschweren. ${ }^{018}$

Die zwischen Server und Spielkopie ausgetauschten Datenpakete können durchaus als „Daten“ i. S. d. § 202a Abs. 2 StGB eingeordnet werden. ${ }^{1019}$ Die Strafbarkeit wird jedoch teilweise bereits wegen Fehlens einer Zugangssicherung auszuschließen sein. Zwar werden die Datenpakete - wie in Kapitel 1 erläutert - zum Teil verschlüsselt, jedoch muss dies nicht immer so sein.

Eine Strafbarkeit gemäß § 202a Abs. 1 StGB scheidet aber jedenfalls deshalb aus, weil die von den Servern des Supportverpflichteten ausgesandten Datenpakete für alle berechtigten Inhaber einer Spielkopie bestimmt sind. Daran ändert auch nichts die Tatsache, dass der Inhaber der Spielkopie die Datenpakete in einem gesonderten Programm näher untersucht, um die Spezifikationen des Kommunikationsprotokolls zu

\footnotetext{
1012 Kindhäuser/Neumann/Paeffgen/Kargl, § 202a StGB, Rn. 4; MüKo StGB/Graf, § 202a StGB, Rn. 12.

1013 Vgl. BeckOK StGB/Weidemann, § 202a StGB, Rn. 4.

1014 Kindhäuser/Neumann/Paeffgen/Kargl, § 202a StGB, Rn. 6.

1015 BeckOK StGB/Weidemann, § 202a StGB, Rn. 8 mwN.

1016 Ebd..

1017 Kindhäuser/Neumann/Paeffgen/Kargl, § 202a StGB, Rn. 7.

1018 BGH, NJW 2015, 3463, 3464 - Bitcoin-Generierung.

1019 Vgl. auch Bedner, Rechtmäßigkeit der "Deep Packet Inspection" (2009), S. 21, abrufbar unter https://kobra.uni-

kassel.de/bitstream/handle/123456789/2009113031192/BednerDeepPacketInspection.pdf?seque nce $=5$ \& is Allowed $=y$.
} 
ermitteln. Für die Strafbarkeit nach § 202a Abs. 1 StGB kommt es allein auf das $Z u$ griffsrecht an den Daten und nicht darüber hinaus auch auf etwaige Nutzungsbeschränkungen in Bezug auf diese Daten an. ${ }^{1020}$

Mit Blick auf die Datenpakete, welche von der Spielkopie in Richtung des Masterservers ausgesandt werden, ist eine Strafbarkeit ebenfalls zu verneinen. Denn über diese Daten hat der Täter bereits selbst die Verfügungsmacht inne und ist damit Berechtigter i. S. d. § 202a Abs. 1 StGB.

\section{b) Strafbarkeit gemäß § 202b StGB und § 303a Abs. 1 StGB}

$\S 202 b$ StGB stellt das unbefugte Sichverschaffen von Daten aus einer nichtöffentlichen Datenübermittlung oder aus der elektromagnetischen Abstrahlung einer Datenverarbeitungsanlage unter Strafe.

Zwar ist es im Gegensatz zu $§ 202 \mathrm{a}$ Abs. 2 StGB nicht erforderlich, dass die Daten einer besonderen Zugangssicherung unterliegen. Wie auch bei § 202a Abs. 1 StGB ist jedoch Tatbestandsvoraussetzung, dass die Daten nicht für den Täter bestimmt sind. Dieses Tatbestandsmerkmal ist nicht anders auszulegen als im Rahmen des $\S 202 \mathrm{a}$ Abs. 1 StGB. ${ }^{1021}$

Da die Datenpakete nach dem Willen des Supportverpflichteten an den Erwerber der Spielkopie übermittelt werden sollen, sind die Daten bereits aus diesem Grund für den Erwerber bestimmt. Das Gleiche gilt auch für die von der Spielkopie ausgesandten Datenpakete, da der Inhaber der Spielkopie zugleich auch Inhaber der Verfügungsmacht über die Daten und daher Berechtigter ist.

Aus diesem Grund ist auch eine Strafbarkeit gemäß § 202b StGB in der Regel nicht gegeben.

$\S 303$ a Abs. 1 StGB stellt das rechtswidrige Löschen, Unterdrücken, Unbrauchbarmachen und Verändern von Daten im Sinne des § 202a Abs. 2 StGB unter Strafe.

Die Veränderung von Daten meint eine ,inhaltliche Umgestaltung“ der Daten, sodass sie einen neuen Informationsgehalt erhalten. ${ }^{1022}$ Nicht erfasst wird die Veränderung der äußeren Form der Daten. ${ }^{1023}$ Aus diesem Grund stellt auch die Entschlüsselung chiffrierter Datenpakete keine Veränderung gemäß § 303a Abs. 1 StGB dar. Der Inhalt der Daten ändert sich schließlich nicht - sie werden lediglich in eine menschenlesbare Form übertragen.

Wie in Kapitel 1 erläutert, werden die vom Masterserver bzw. die von der Spielkopie ausgesandten Datenpakete vom Inhaber der Spielkopie, welcher die Spezifikationen des Kommunikationsprotokolls herauszufinden versucht, nicht nur abgefangen, son-

\footnotetext{
1020 Kindhäuser/Neumann/Paeffgen/Kargl, § 202a StGB, Rn. 8.

1021 Vgl. Kindhäuser/Neumann/Paeffgen/Kargl, § 202b StGB, Rn. 4.

1022 BeckOK StGB/Weidemann, § 303a StGB, Rn. 14.

${ }^{1023}$ MüKo StGB/Wieck-Noodt, § 303a StGB, Rn. 15.
} 
dern - falls sie verschlüsselt worden sind - auch entschlüsselt und in ein lesbares Format übertragen ${ }^{1024}$. Eine strafbare Veränderung von Daten gemäß § 303a Abs. 1 StGB ist darin jedoch nicht zu erblicken.

\section{Unzulässige Erlangung eines Betriebsgeheimnisses}

Weiterhin kann dem Reverse Engineering ein Schutz des Kommunikationsprotokolls als Geschäftsgeheimnis entgegenstehen. Seit dem 5. Juli 2016 ist die Richtlinie (EU) 2016/943 über den Schutz vertraulichen Know-hows und vertraulicher Geschäftsinformationen (Geschäftsgeheimnisse) vor rechtswidrigem Erwerb sowie rechtswidriger Nutzung und Offenlegung (im Folgenden: „Geschäftsgeheimnis-Rl.“) in Kraft. Der deutsche Gesetzgeber hat die Richtlinie mit dem Geschäftsgeheimnisschutzgesetz (GeschGehG), welches am 26.04.2019 in Kraft getreten ist, umgesetzt. Vor dem Hintergrund, dass Geschäftsgeheimnisse bisher nicht in jedem Mitgliedsstaat der EU gleichermaßen geschützt waren, dient die Geschäftsgeheimnis-Rl. dazu, Regelungen zum Schutz von Geschäftsgeheimnissen in den Mitgliedsstaaten zu vereinheitlichen. ${ }^{1025}$ Bis zum Inkrafttreten des GeschGehG war der Schutz von Geschäftsgeheimnissen nicht in einem einheitlichen Gesetz konzentriert, sondern nur punktuell in verschiedenen Gesetzen, z. B. in den $\S \S 17-19$ UWG, geregelt. ${ }^{1026}$

Im Folgenden wird untersucht, ob das Kommunikationsprotokoll als Betriebs- bzw. Geschäftsgeheimnis geschützt ist und ob dieser Schutz dem Reverse Engineering des Protokolls entgegensteht. Dies wird sowohl nach alter (a)) als auch nach aktueller (b)) Rechtslage unter Geltung der Richtlinie und dem GeschGehG geprüft.

\section{a) Alte Rechtslage}

\section{aa) Schutz als Betriebsgeheimnis}

Betriebs- und Geschäftsgeheimnisse sind Tatsachen, welche einen Unternehmensbezug aufweisen, nicht offenkundig sind und deren Geheimhaltung von einem Geheimhaltungswillen und -interesse des Unternehmens- bzw. Betriebsinhabers getragen wird. ${ }^{1027}$ Der Unternehmensbezug der Tatsache setzt voraus, dass sie im Zusammenhang mit dem Unternehmen steht und nicht etwa der Privatsphäre des Unternehmers entstammt. ${ }^{1028}$

Die Nichtoffenkundigkeit der Tatsache ist zu bejahen, wenn sie weder ,allgemein bekannt“ noch „leicht zugänglich“ ist. ${ }^{1029}$ Die Tatsache ist leicht zugänglich, wenn sich

\footnotetext{
1024 Vgl. zi, Toorcon: San Diego (2014), ab Min. 17:30, abrufbar unter https://www.youtube.com/watch?v=K4dyyLpMkQk.

1025 Vgl. Erwg. 10 der Geschäftsgeheimnisse-RL.

1026 von Busekist/Racky, ZRP 2018, 135, 136.

1027 BGH, GRUR 2009, 603, 604 - Versicherungsuntervertreter; Ohly/Sosnitza/Ohly, § 17 UWG, Rn. 5; Schweyer, Reverse Engineering, S. 458; Harte-Bavendamm, GRUR 1990, 657, 660.

1028 Köhler/Bornkamm/Feddersen/Köhler, § 17 UWG, Rn. 5.

1029 Ebd., Rn. 6.
} 
jeder Interessierte von ihr ,ohne größere Schwierigkeiten und Opfer mit lauteren Mitteln Kenntnis verschaffen kann“. ${ }^{1030}$

Das Geheimhaltungsinteresse des Unternehmers liegt vor, wenn das Bekanntwerden der Tatsache den Wettbewerb des geheim haltenden Unternehmens gefährden und im Gegenzug den Wettbewerb anderer Unternehmen begünstigen kann. ${ }^{1031}$ Der Geheimhaltungswille des Unternehmensinhabers kann entweder ausdrücklich bekundet werden, oder sich aus den Umständen ergeben, etwa weil die Tatsache für den Geschäftsbetrieb und seine Position am Markt von Bedeutung ist und der Unternehmensinhaber sie daher geheim halten würde. ${ }^{1032}$

Unter Berücksichtigung dieser Kriterien können Kommunikationsprotokolle im Einzelfall durchaus als Betriebsgeheimnisse eingeordnet werden. Der Unternehmensbezug und die Nichtoffenkundigkeit liegen regelmäßig vor. Es ist in Kapitel 1 außerdem dargestellt worden, wie aufwendig und anspruchsvoll die Ermittlung des Kommunikationsprotokolls sein kann. ${ }^{1033}$ Die hierfür aufzuwendenden, nicht unerheblichen Ressourcen dürften die von der Rechtsprechung und Literatur festgelegte Opfergrenze regelmäßig überschreiten. $\mathrm{Zu}$ beachten ist dabei allerdings, dass das Kommunikationsprotokoll dann nicht (mehr) als Betriebsgeheimnis angesehen werden kann, wenn hierfür ein Patent erteilt worden ist. Grundsätzlich kann nämlich jedermann gemäß § 31 Abs. 1 Satz 1 PatG Einsicht in die Akten von erteilten Patenten nehmen ${ }^{1034}$, was die Nichtoffenkundigkeit einer Tatsache ausschlie $\beta \mathrm{t}^{1035}$.

Darüber hinaus kann das Kommunikationsprotokoll auch aus wettbewerblicher Sicht für den Publisher wirtschaftlich wertvoll sein. So ist es möglich, dass ein bestimmtes Protokoll beispielsweise einen besonders effektiven und schnellen Informationsaustausch zwischen Spielkopie und Server ermöglicht und damit zu einem flüssigeren Spielerlebnis führt, was einem Unternehmen einen entscheidenden Wettbewerbsvorteil gegenüber Konkurrenten geben kann. Aus diesem Grund kann im Einzelfall sowohl ein Geheimhaltungsinteresse als auch -wille bejaht werden.

\section{bb) Verstoß gegen § 17 UWG}

Das Reverse Engineering des als Betriebsgeheimnisses geschützten Kommunikationsprotokolls könnte gegen $\S 17$ Abs. 2 UWG verstoßen.

$\S 17$ Abs. 2 Nr. 1 UWG stellt das mit bestimmten Mitteln vorgenommene unbefugte Sichverschaffen oder Sichern eines Geschäfts- oder Betriebsgeheimnisses durch einen Arbeitnehmer oder einen Dritten unter Strafe. § 17 Abs. 2 Nr. 2 UWG bestraft darüber

\footnotetext{
${ }^{1030}$ Ebd., Rn. 8; vgl. BayObLG, GRUR 1991, 694, 695 - Geldspielautomat.

1031 Köhler/Bornkamm/Feddersen/Köhler, § 17 UWG, Rn. 9.

${ }^{1032}$ Harte-Bavendamm/Henning-Bodewig/Harte-Bavendamm, 17 UWG, Rn. 5; vgl. BGH, GRUR 2006, 1044, 1046, Tz. 19 - Kundendatenprogramm.

${ }^{1033}$ Siehe dazu oben, S. 28 ff.

${ }^{1034} \mathrm{Mes} / \mathrm{Mes}, \S 31 \mathrm{PatG}$, Rn. 6 f.

1035 So auch in Bezug auf Patentanmeldungen entsprechend § 31 Abs. 2 PatG Köhler/Bornkamm/Feddersen/Köhler, § 17 UWG, Rn. 7.
} 
hinaus auch das sonstige unbefugte Sichverschaffen oder Sichern eines Geschäfts - oder Betriebsgeheimnisses, sofern dieses unbefugt verwertet oder jemandem mitgeteilt wird.

Das „Sichverschaffen“ meint die aktive Kenntniserlangung vom Geheimnis durch den Täter und das „Sichern“ die Schaffung einer bleibenden Kenntnis hiervon. ${ }^{1036}$ Das Sichverschaffen oder Sichern kann nach $\S 17$ Abs. 2 Nr. 1 lit. a) UWG durch die „Anwendung technischer Mittel“ erfolgen, worunter der Einsatz jeglicher Vorrichtungen $\mathrm{zu}$ verstehen ist, die zur Kenntniserlangung von Geheimnissen dienen können. ${ }^{1037}$ Diese Handlungen sind unbefugt, wenn sie nicht durch Rechtfertigungsgründe gedeckt sind. ${ }^{1038}$

Einer der - zum subjektiven Tatbestand zuzuordnenden - Zwecke, der mit dem Sichverschaffen oder Sichern des Geheimnisses verfolgt werden muss, ist der Eigennutz, worunter das Streben nach einem materiellen oder zumindest immateriellen Vorteil verstanden wird. ${ }^{1039}$ Der immaterielle Vorteil muss mit einem materiellen Vorteil jedoch vergleichbar sein. ${ }^{1040}$ Das Sichverschaffen oder Sichern des Betriebsgeheimnisses wird darüber hinaus auch dann von der Vorschrift erfasst, wenn einem Dritten dieser Vorteil zugeführt werden soll. ${ }^{1041}$

Die Literatur sieht das Reverse Engineering überwiegend als von $\S 17$ Abs. $2 \mathrm{Nr}$. $1^{1042}$ bzw. Nr. $2^{1043}$ UWG erfasst an. ${ }^{1044}$

Dementsprechend stellt die in Kapitel 1 beschriebene Kenntniserlangung von den Spezifikationen des Kommunikationsprotokolls im Wege des Reverse Engineering ein Sichverschaffen eines Betriebsgeheimnisses unter Anwendung technischer Mittel gemäß $\S 17$ Abs. 2 Nr. 1 lit. a) UWG dar. Der subjektive Tatbestand des Eigennutzes bzw. der Drittbegünstigung ist gleichfalls erfüllt, da die Kenntnis vom Kommunikationsprotokoll grundsätzlich allen Spielern die Entwicklung oder den Einsatz eines eigenen Masterservers erlaubt, mithilfe dessen der Online-Mehrspielermodus wiederhergestellt werden kann. Daher stellt das Streben nach der Kenntnis des Kommunikationsprotokolls auch ein Streben nach einem materiellen Vorteil dar.

\footnotetext{
1036 Harte-Bavendamm/Henning-Bodewig/Harte-Bavendamm, § 17 UWG, Rn. 20.

1037 Ebd., Rn. 22; Kochmann, Schutz des „Know-how“, S. 118.

1038 Ohly/Sosnitza/Ohly, § 17 UWG, Rn. 19.

1039 Köhler/Bornkamm/Feddersen/Köhler, § 17 UWG, Rn. 25; Ohly/Sosnitza/Ohly, § 17 UWG, Rn. 25; Harte-Bavendamm/Henning-Bodewig/Harte-Bavendamm, § 17 UWG, Rn. 15.

1040 Ohly/Sosnitza/Ohly, § 17 UWG, Rn. 25.

1041 Ebd.

${ }^{1042}$ Kalbfus, Know-how-Schutz, Rn. 545; ders., GRUR 2016, 1009, 1012; Ohly/Sosnitza/Ohly, § 17 UWG, Rn. 26a; Ohly, GRUR 2014, 1, 7; Wiebe, CR 1992, 134, 137; MüKo UWG/Brammsen, § 17 UWG, Rn. 88; Baranowski/Glaßl, BB 2016, 2563, 2566.

${ }^{1043}$ Kalbfus, Know-how-Schutz, Rn. 546; Wiebe, CR 1992, 134, 137; Köhler/Bornkamm/Feddersen/Köhler, § 17 UWG, Rn. 33.

1044 Vgl. auch BGH, GRUR 2003, 356, 358 - Präzisionsmessgeräte.
} 
$\mathrm{Zu}$ beachten ist weiterhin, dass die etwaige Veröffentlichung von Informationen über das Kommunikationsprotokoll beispielsweise auf einer Internetseite ein „Mitteilen“ im Sinne des $§ 17$ Abs. 2 Nr. 2 UWG darstellen kann.

Das Reverse Engineering zu wissenschaftlichen Zwecken soll demgegenüber vom Tatbestand des $\S 17$ Abs. 2 UWG ausgenommen sein. ${ }^{1045}$ Daher könnte man annehmen, dass das von Wissenschaftlern aus dem Bereich der Game Studies oder von Gedächtnisinstitutionen vorgenommene Reverse Engineering des Kommunikationsprotokolls nicht strafbar ist nach $\S 17$ Abs. 2 UWG. Zu beachten ist dabei allerdings, dass sich das Reverse Engineering hier nicht nur im Interesse an der Kenntnis von den Spezifikationen des Kommunikationsprotokolls erschöpft, sondern gerade deshalb vorgenommen wird, um den Online-Mehrspielermodus wiederherzustellen. Auch Wissenschaftler und Gedächtnisinstitutionen erstreben insofern einen materiellen Vorteil. Aus diesem Grund sind auch von Wissenschaftlern und Gedächtnisinstitutionen vorgeneommene Rückentwicklungen strafbewehrt, sofern diese die Wiederherstellung des Online-Mehrspielermodus zum Gegenstand haben.

An der Strafbarkeit des Reverse Engineering nach $\S 17$ Abs. 2 UWG üben verschiedene Literaturstimmen allerdings Kritik. ${ }^{1046}$

Gegen die Strafbarkeit wird vorgebracht, dass durch die Vorschrift des $§ 17$ Abs. 2 UWG ein besonderer wettbewerbsrechtlicher Leistungsschutz für komplexe Güter geschaffen werde, was insbesondere dann problematisch sei, wenn diese Güter ansonsten frei nachgeahmt werden könnten. ${ }^{1047}$ Insofern gehe die Pönalisierung des Reverse Engineering weit über den Schutz betriebsinterner Informationen hinaus. ${ }^{1048}$

Das Reverse Engineering sei außerdem vom Unrechtsgehalt her ein völlig anderes Verhalten als die Betriebsspionage, aus deren Anlass der Gesetzgeber § 17 UWG eigentlich eingeführt habe. ${ }^{1049} \S 17$ Abs. 2 UWG stelle aufgrund der Weite des Tatbestandes mit dem Reverse Engineering auch Tathandlungen unter Strafe, die als solche nicht strafwürdig seien. ${ }^{1050}$

Der Kritik in der Literatur ist zuzustimmen. Es erscheint fragwürdig, dass Tathandlungen, die anhand von rechtmäßig erworbenen Produkten vorgenommen werden und die noch dazu - wie hier - nicht das Ziel haben, Konkurrenzprodukte mithilfe der mit dem Reverse Engineering gewonnenen Erkenntnisse zu entwickeln, strafbar sind. Das Reverse Engineering hat hier lediglich den Zweck, den ursprünglichen Gebrauchszustand der digitalen Spiele zu privaten oder zu Forschungszwecken zu erhalten bzw. wiederherzustellen.

1045 Harte-Bavendamm, GRUR 1990, 657, 663; Ohly/Sosnitza/Ohly, § 17 UWG, Rn. 25.

1046 Schweyer, Reverse Engineering, S. 476 ff.; Ohly, GRUR 2014, 1, 7; Ohly/Sosnitza/ders., § 17 UWG, Rn. 26a; Beater, Unlauterer Wettbewerb, Rn. 1893; Kalbfus, Know-how-Schutz, Rn. 569 ff.; Kuhlmann, CR 1989, 177, 184; Kochmann, Schutz des „Know-how“, S. 135 f.; vgl. auch Harte-Bavendamm, in FS Köhler, S. 244 ff.

1047 Ohly/Sosnitza/Ohly, § 17 UWG, Rn. 26a; Beater, Unlauterer Wettbewerb, Rn. 1893.

1048 Ohly/Sosnitza/Ohly, § 17 UWG, Rn. 26a.

1049 Schweyer, Reverse Engineering, S. 480; vgl. auch Kochmann, Schutz des „Know-how“, S. 135 f.

${ }^{1050}$ Kochmann, Schutz des „Know-how“, S. 135 f. 
Fraglich ist jedoch, auf welchem dogmatischen Wege ein Ausschluss des Reverse Engineerings vom Anwendungsbereich des $\S 17$ Abs. 2 UWG erreicht werden kann.

Einerseits ist es möglich, das Tatbestandsmerkmal „unbefugt“ in $\S 17$ Abs. 2 Nr. 1 und 2 UWG im Zuge einer Interessenabwägung dahingehend auszulegen, dass das Reverse Engineering keine unbefugte Tathandlung mehr darstellt. ${ }^{1051}$ Hiergegen spricht jedoch, dass durch eine Interessenabwägung das Tatbestandsmerkmal „unbefugt“ in hohem Maße unbestimmt würde und dazu nicht immer sichergestellt wäre, dass die Interessenabwägung auch zugunsten des Täters ausfiele. ${ }^{1052}$ Dies ist mit dem Bestimmtheitsgebot des Art. 103 Abs. 2 GG nicht in Einklang zu bringen. ${ }^{1053}$

Überzeugender ist dagegen der Ausschluss des Reverse Engineerings im Wege einer teleologischen Reduktion des $\S 17$ Abs. 2 UWG mit der Folge, dass diese Vorschrift auf das Reverse Engineering von rechtmäßig in Verkehr gebrachten Produkten, die der Reverse Engineerende zulässigerweise besitzt, keine Anwendung findet. ${ }^{1054}$ Dies muss insbesondere dann gelten, wenn das Reverse Engineering keinen wettbewerblichen Zwecken dient, wie etwa der Herstellung eines Konkurrenzproduktes, sondern der Erhaltung bzw. Wiederherstellung der Nutzungsmöglichkeit des Produkts.

Das Reverse Engineering des Kommunikationsprotokolls ist damit aufgrund einer teleologischen Reduktion des $§ 17$ Abs. 2 UWG nicht strafbar, wenn die Untersuchung des Protokolls anhand einer rechtmäßig in Verkehr gebrachten Spielkopie erfolgt, welche der Untersuchende auch zulässigerweise besitzen darf, und sofern das Reverse Engineering mit dem Ziel vorgenommen wird, den Online-Mehrspielermodus wiederherzustellen.

\section{cc) Verwirklichung zivilrechtlicher Unterlassens- und Schadenser- satzansprüche}

\section{(1) Deliktsrechtliche Ansprüche}

Sowohl die ältere Rechtsprechung als auch ein Teil der Literatur vertreten die Ansicht, dass Betriebs- und Geschäftsgeheimnisse Schutz nach $\S 823$ Abs. 1 BGB genieBen. ${ }^{1055}$ Die Folge dieser Auffassung ist, dass der Inhaber der Geschäfts- und Betriebsgeheimnisse gegen denjenigen aus § 823 Abs. 1 BGB Schadensersatz oder in Verbindung mit $\S 1004$ Satz 1 BGB analog Beseitigung ${ }^{1056}$ oder Unterlassung verlangen

\footnotetext{
1051 Ohly/Sosnitza/Ohly, § 17 UWG, Rn. 26a; Ohly, GRUR 2014, 1, 7.

1052 Kalbfus, Know-how-Schutz, Rn. 573.

1053 Ebd.; Kochmann, Schutz des „Know-how“, S. 138.

1054 Vgl. Kalbfus, Know-how-Schutz, Rn. 574.

1055 BGH, GRUR 1955, 388, 389 - Dücko; GRUR 1955, 468, 472 - Schwermetall-Kokillenguß; NJW 1990, 52, 53 - Forschungskosten; Nastelski, GRUR 1957, 1, 6; Mes, GRUR 1979, 584, 592; Habel, CR 1991, 257, 258; Enders, GRUR 2012, 25, 28; Ohly/Sosnitza/Ohly, § 17 UWG, Rn. 49; Hk-BGB/Staudinger, § 823 BGB, Rn. 120; Köhler/Bornkamm/Feddersen/Köhler, § 17 UWG, Rn. 53.

1056 Z. B. die Vernichtung von Aufzeichnungen des Geheimnisses, vgl. Kalbfus, Know-how-Schutz, Rn. 439.
} 
kann, der die Geschäfts- und Betriebsgeheimnisse im Wege des Reverse Engineering ermittelt, verwertet oder anderen mitteilt. ${ }^{1057}$

Zum Teil werden die Betriebs- und Geschäftsgeheimnisse dabei unmittelbar als ein „sonstiges Recht“ im Sinne des $\S 823$ Abs. 1 BGB eingeordnet. ${ }^{1058}$ Zur Begründung dafür wird vorgebracht, dass die Rechtsordnung den Betriebs- und Geschäftsgeheimnissen einen strafrechtlichen Schutz beimisst, was sowohl die hohe Bedeutung von Betriebs- und Geschäftsgeheimnissen als auch die Notwendigkeit verdeutliche, diese als sonstiges Recht im Sinne des $\S 823$ Abs. 1 BGB zu schützen. ${ }^{1059}$ Darüber hinaus habe der Inhaber der Geschäfts- und Betriebsgeheimnisse eine Dispositionsbefugnis inne, welche derjenigen von Urhebern und Patentinhabern nahekomme, da er Dritte von der Nutzung der Geheimnisse ausschließen oder ihnen eine Lizenz zur Nutzung der Geheimnisse einräumen könne. ${ }^{1060}$ Dabei sei das sonstige Recht an den Betriebsund Geschäftsgeheimnissen als ein Rahmenrecht ausgestaltet, was zur Folge habe, dass die Verwertung der Geheimnisse noch nicht die Rechtswidrigkeit indiziere, welche vielmehr erst aufgrund einer gesonderten Interessenabwägung festzustellen sei. ${ }^{1061}$

Ein anderes Meinungsspektrum sieht Betriebs- und Geschäftsgeheimnisse zwar nicht unmittelbar als ein „sonstiges Recht“ im Sinne des § 823 Abs. 1 BGB an, jedoch als Bestandteil des Rechts am eingerichteten und ausgeübten Gewerbebetrieb, welches ebenfalls nach $\S 823$ Abs. 1 BGB als sonstiges Recht Schutz genießt. ${ }^{1062}$

Die neuere Literatur lehnt dagegen einen Schutz der Betriebs- und Geschäftsgeheimnisse nach $\S 823$ Abs. 1 BGB ab. ${ }^{1063}$ Begründet wird dies einerseits damit, dass es Betriebs- und Geschäftsgeheimnissen an dem für sonstige Rechte im Sinne des $\S 823$ Abs. 1 BGB erforderlichen „Zuweisungsgehalt“ fehle. ${ }^{1064}$ Der Rechtsordnung lasse sich also keine Zuordnung der Geheimnisse zu einem bestimmten Inhaber entnehmen. ${ }^{1065}$ Denn nicht die Monopolisierung eines Geheimnisses werde geschützt, sondern der Geheimnisinhaber werde vor bestimmten, rechtlich missbilligten Zugriffen auf seine Geheimnisse bewahrt. ${ }^{1066}$

\footnotetext{
1057 Vgl. Ohly/Sosnitza/Ohly, § 17 UWG, Rn. 48 f.

1058 Ebd., Rn. 49; Mes, GRUR 1979, 584, 592; Köhler/Bornkamm/Feddersen/Köhler, § 17 UWG, Rn. 53.

1059 Mes, GRUR 1979, 584, 592.

1060 Ohly/Sosnitza/Ohly, Vorbemerkungen vor $\S$ 17-19 UWG, Rn. 4.

1061 Ohly/Sosnitza/Ohly, § 17 UWG, Rn. 49; Köhler/Bornkamm/Feddersen/Köhler, § 17 UWG, Rn. 53.

1062 BGH, GRUR 1955, 468, 472; NJW 1990, 52, 53; Habel, CR 1991, 257, 258; Hk-BGB/Staudinger, § 823 BGB, Rn. 120; Enders, GRUR 2012, 25, 28.

1063 Kochmann, Schutz des „Know-how“, S. 237; Kalbfus, Know-how-Schutz, Rn. 410; Erman/Wilhelmi, § 823 BGB, Rn. 55; MüKo UWG/Brammsen, § 17 UWG, Rn. 7; MüKo BGB/Wagner, § 823 BGB, Rn. 284.

1064 Kalbfus, Know-how-Schutz, Rn. 407 ff.; Erman/Wilhelmi, § 823 BGB, Rn. 55; Habel, CR 1991, $257,258$.

1065 Kalbfus, Know-how-Schutz, Rn. 408.

1066 Ebd.
} 
Zum anderen liefe ein Schutz der Betriebs- und Geschäftsgeheimnisse als ,,sonstiges Recht" der für Immaterialgüterrechte teilweise geltenden Pflicht zur Offenlegung des geschützten Gegenstandes zuwider. ${ }^{1067}$ Darüber hinaus würde der Geheimnisschutz im Gegensatz etwa zum Patentschutz - zeitlich unbegrenzt gewährt. ${ }^{1068}$ Das hätte jedoch zur Folge, dass Forschung und wissenschaftlicher Fortschritt mangels Offenlegung von Wissen behindert werden würden. ${ }^{1069}$

Auch können Betriebs- und Geschäftsgeheimnisse nach dieser Ansicht nicht vom Recht am eingerichteten und ausgeübten Gewerbebetrieb umfasst sein, da es am betriebsbezogenen Eingriff fehle. ${ }^{1070}$ Das Wissen kann schließlich auch noch nach seiner Aufdeckung verwendet werden und berührt den Betrieb damit in aller Regel nicht. ${ }^{1071}$

Der letztgenannten Auffassung ist zu folgen. Der Rechtsschutz der Inhaber von Betriebs- und Geschäftsgeheimnissen hat keine vergleichbar umfassende Regelung erfahren wie der von Inhabern der Immaterialgüterrechte, wie z. B. das Urheberrecht oder das Patent. Lediglich bestimmte Arten von Zugriffen auf und Verwertungen von Betriebs- und Geschäftsgeheimnissen sind strafbewehrt. Ein Schutz der Betriebs- und Geschäftsgeheimnisse als sonstiges Recht nach § 823 Abs. 1 BGB wäre daher nicht gerechtfertigt. Damit stehen dem Inhaber des Geschäfts- und Betriebsgeheimnisses keine Ansprüche aus $\S 823$ Abs. 1 BGB zu, falls dieses im Wege des Reverse Engineerings offengelegt und zur Wiederherstellung der Nutzungsmöglichkeit des OnlineMehrspielermodus verwendet wird.

\section{(2) Ansprüche aus Bereicherungsrecht und einer unechten Geschäftsfüh- rung ohne Auftrag}

Umstritten ist darüber hinaus, ob dem Inhaber des Betriebs- oder Geschäftsgeheimnisses gegen demjenigen, welcher dieses im Wege des Reverse Engineerings erlangt hat, ein Bereicherungsanspruch gemäß $\S 812$ Abs. 1 Satz 1 Fall 2 BGB zusteht. Nach h. M. setzt die Eingriffskondiktion eine Rechtsposition voraus, welche nach der Rechtsordnung dem Bereicherungsgläubiger zu dessen ausschließlicher Verfügung und Verwertung zugewiesen ist. ${ }^{1072}$

Eine in der Literatur vertretene Ansicht befürwortet einen solchen Zuweisungsgehalt. ${ }^{1073}$ Der Inhaber von Betriebs- und Geschäftsgeheimnissen könne schließlich Drit-

\footnotetext{
1067 Kochmann, Schutz des „Know-how“, S. 228.

1068 Ebd.; vgl. auch Habel, CR 1991, 257, 258.

1069 Kochmann, Schutz des „Know-how“, S. 228.

1070 Ebd., S. 233.

1071 Ebd.

1072 BGH, NJW 2012, 2034, 2037, Tz. 40 - gewinn.de; NJW 2012, 3572, 3574, Tz. 9 - Mietauskehrstreit; NJW 1990, 52, 52; Palandt/Sprau, § 812 BGB, Rn. 40; Erman/Buck-Heeb, § 812 BGB, Rn. 65 mwN.

1073 Köhler/Bornkamm/Feddersen/Köhler, § 17 UWG, Rn. 55; Ohly/Sosnitza/Ohly, § 17 UWG, Rn. 50; BeckOK BGB/Wendehorst, § 812 BGB, Rn. 130; MüKo BGB/Schwab, § 812 BGB, Rn.
} 
ten die Nutzung des Geheimnisses untersagen oder auch gegen ein Entgelt gestatten. ${ }^{1074}$ Bei den Betriebs- und Geschäftsgeheimnissen handle es sich außerdem um Vermögenswerte, die über den allgemeinen lauterkeitsrechtlichen Schutz hinaus durch besondere Strafvorschriften ( $\S 17,18$ UWG) geschützt werden. ${ }^{1075}$

Die überzeugenderen Argumente sprechen allerdings gegen die Anerkennung eines Zuweisungsgehalts und damit gegen einen bereicherungsrechtlichen Schutz der Betriebs- und Geschäftsgeheimnisse. ${ }^{1076}$ Zwar bieten die $\S \S 17,18$ UWG einen Schutz gegen bestimmte Arten der Erlangung von Betriebs- und Geschäftsgeheimnissen. Dieser letztlich aber nur lückenhafte Rechtsschutz dient vornehmlich dazu, den Aufwand des Geheimnisinhabers mit Blick auf die von ihm vorgenommenen Schutzmaßnahmen zu reduzieren, nicht jedoch, ihm eine Alleinstellung hinsichtlich seiner Betriebs- und Geschäftsgeheimnisse zu sichern. ${ }^{1077} \mathrm{Zu}$ beachten ist auch, dass die Eingriffkondiktion - im Gegensatz zu den $\S \S 17,18$ UWG - nicht danach unterscheidet, auf welche Art und Weise die Geheimnisse erlangt werden ${ }^{1078}$, sodass der Geheimnisinhaber stets kondizieren könnte, unabhängig davon, ob die Erlangung der Geheimnisse im konkreten Fall gemäß $\S 17,18$ UWG strafbar ist oder nicht. Damit würden die gesetzgeberischen Wertungen unterlaufen, die eben nur bestimmte Formen der Geheimniserlangung für unzulässig erklären.

Damit scheidet auch ein Anspruch gemäß § 812 Abs. 1 Satz 1 Fall 2 BGB gegen denjenigen aus, welcher das Betriebs- oder Geschäftsgeheimnis im Wege des Reverse Engineering ermittelt und verwertet.

Aus den gleichen Gründen, die zur Ablehnung eines Kondiktionsanspruchs aus $\S$ 812 Abs. 1 Satz 1 Fall 2 BGB geführt haben, ist auch ein Anspruch gemäß $§ 687$ Abs. 2 BGB wegen angemaßter Eigengeschäftsführung mit Blick auf das Reverse Engineering von Betriebs- und Geschäftsgeheimnissen zu verneinen. Es fehlt auch hier an einer Rechtsposition, die dem Anspruchsgläubiger mit ausschließlicher Wirkung zugewiesen ist. ${ }^{1079}$ Damit fehlt es schon an einem objektiv fremden Geschäft. ${ }^{1080}$

\section{b) Aktuelle Rechtslage}

Die 2016 in Kraft getretene Geschäftsgeheimnis-Rl. dient - wie bereits dargestellt der Vereinheitlichung von Regelungen zum Schutz von Geschäftsgeheimnissen in der

307; MüKo UWG/Fritzsche, § 9 UWG, Rn. 125; Loewenheim, WRP 1997, 913, 916;

Kiethe/Groeschke, WRP 2005, 1358, 1362; Siems, WRP 2007, 1146, 1147.

1074 Loewenheim, WRP 1997, 913, 916.

1075 MüKo UWG/Fritzsche, § 9 UWG, Rn. 125.

1076 Vgl. Kochmann, Schutz des „Know-how“, S. 246; Kalbfus, Know-how-Schutz, Rn. 281 ff.; Ellger, Bereicherung, S. 402; Peukert, Güterzuordnung, S. 821.

1077 Kalbfus, Know-how-Schutz, Rn. 487; Peukert, Güterzuordnung, S. 821.

1078 Kalbfus, Know-how-Schutz, Rn. 489.

1079 Ebd., Rn. 493; vgl. auch Kochmann, Schutz des „Know-how“, S. 248 f.; a. A. wohl, jedoch ohne Begründung BGH, GRUR 2012, 1048, 1049, Tz. 27 - MOVICOL-Zulassungsantrag; Köhler/Bornkamm/Feddersen/Köhler, § 17 UWG, Rn. 56; Ohly/Sosnitza/Ohly, § 17 UWG, Rn. 50.

1080 Kalbfus, Know-how-Schutz, Rn. 493. 
Europäischen Union. Die Mitgliedsstaaten dürfen allerdings innerhalb der Grenzen des Art. 1 Abs. 1 Satz 2 Geschäftsgeheimnis-Rl. einen weitergehenden Schutz für Geschäftsgeheimnisse vorsehen. Insofern sieht die Geschäftsgeheimnis-Rl. nur einen Mindestschutz vor. ${ }^{1081}$

Der in Art. 2 Nr. 1 der Geschäftsgeheimnis-RL definierte Geschäftsgeheimnis-Begriff weicht von der oben erläuterten Definition ${ }^{1082}$ insofern ab, als die geschützten Informationen Gegenstand von Geheimhaltungsmaßnahmen sein müssen. ${ }^{1083}$ Ein solches Merkmal war dem deutschen Begriffsverständnis bisher fremd. ${ }^{1084}$ An solche Maßnahmen, wozu beispielsweise vertragliche Vertraulichkeitsverpflichtungen gehören, sind jedoch grundsätzlich keine allzu hohen Anforderungen zu stellen. ${ }^{1085}$

Sofern Publisher entsprechende Maßnahmen zur Geheimhaltung ihrer Kommunikationsprotokolle implementieren, sind sie auch nach zukünftiger Rechtslage geschützt.

$\mathrm{Zu}$ beachten ist jedoch die Regelung des Art. 3 Abs. 1 lit. b) Var. 1 der Geschäftsgeheimnis-Rl., welcher das Reverse Engineering öffentlich verfügbar gemachter Produkte ausdrücklich für zulässig erklärt. Die Mitgliedsstaaten dürfen gemäß Art. 1 Abs. 1 Satz 2 Geschäftsgeheimnis-Rl. auch keine Vorschriften erlassen, welche das Reverse Engineering solcher Produkte für unzulässig erklären. Eine Ausnahme gilt allerdings für nicht öffentlich verfügbar gemachte Produkte ( 3 Abs. 1 lit. b) Var. 2). Seinem Vertragspartner kann der Inhaber des Geschäftsgeheimnisses das Reverse Engineering des zur Verfügung gestellten, nicht öffentlich verfügbar gemachten Produkts vertraglich untersagen.

Digitale Spiele stellen öffentlich verfügbar gemachte Produkte dar, sodass das Reverse Engineering ihrer Kommunikationsprotokolle aufgrund der Geschäftsgeheimnis-Rl. zulässig ist. Da die Mitgliedsstaaten keine Vorschriften erlassen dürfen, welche das Reverse Engineering öffentlich verfügbar gemachter Produkte verbieten, erübrigt sich nach neuer Rechtslage auch die Diskussion zur Frage, ob der Inhaber des Betriebsgeheimnisses im Falle des Reverse Engineering zivilrechtliche Ansprüche nach dem Delikts- oder Bereicherungsrecht geltend machen kann. Solche Ansprüche bestehen nunmehr - wie auch schon nach einer alten, jedoch umstrittenen Rechtsansicht ${ }^{1086}$ - nicht. Dies ergibt sich mit Blick auf $\S 823$ Abs. 1 BGB und dem Schutz „,sonstiger Rechte" auch schon daraus, dass die Richtlinie keine Schaffung eines absoluten Rechts bezweckt. ${ }^{1087}$

\footnotetext{
1081 Kalbfus, GRUR 2016, 1009, 1010.

1082 Siehe oben, S. $188 \mathrm{f}$.

1083 Baranowski/Glaßl, BB 2016, 2563, 2565.

1084 Gärtner, NZG 2014, 650, 651; Kalbfus, GRUR-Prax 2017, 391, 391.

1085 Ausführlich hierzu Kalbfus, GRUR-Prax 2017, 391, 392 f.; Voigt/Herrmann/Grabenschröer, BB 2019, 142, $144 \mathrm{ff}$.

1086 Siehe dazu oben, S. 192 ff.

1087 Vgl. dazu Kalbfus, GRUR 2016, 1009, 1012; vgl. dazu auch die Begründung zum Entwurf des GeschGehG, BT-Drs. 19/4724, S. 28.
} 
Das GeschGehG setzt die Geschäftsgeheimnis-R1. nicht durch punktuelle Änderungen bereits bestehender nationaler Gesetze, sondern durch Einführung eines einheitlichen Spezialgesetzes um. ${ }^{1088}$

Das Gesetz hat auch einen neuen Straftatbestand (§ 23) eingeführt, welcher die bisherigen Strafvorschriften des UWG, und damit auch § 17 UWG, ersetzen soll. ${ }^{1089}$ Das Reverse Engineering ist entsprechend der Geschäftsgeheimnis-Rl. in $\S 3$ Abs. 1 Nr. 2 GeschGehG als erlaubte Handlung geregelt. ${ }^{1090}$ Da nach $\S 23$ GeschGehG nur verbotene Handlungen nach $\S 4$ GeschGehG strafbar sind, ist das Reverse Engineering als erlaubte Handlung auch im nationalen Recht nicht strafbar.

\section{Reverse Engineering der Payloads}

Die Payloads sind die eigentlichen Nutzdaten innerhalb der zwischen der Spielkopie und dem Server ausgetauschten Datenpakete und beinhalten bestimmte programmbezogene Informationen und Anweisungen. ${ }^{1091}$ Sie unterscheiden sich daher vom Kommunikationsprotokoll, welches eine Konvention über den Austausch von Daten zwischen zwei Bestandteilen eines Computersystems darstellt.

Trotz dieses Unterschieds sind auch die Payloads in hohem Maße - wie auch das Kommunikationsprotokoll - funktional. Ein urheberrechtlicher Schutz der Payloads scheidet daher genauso wie für das Kommunikationsprotokoll aus. Dagegen kann im Einzelfall ein patentrechtlicher Schutz sowie ein Schutz des Payloads als Geschäftsgeheimnis in Betracht kommen.

Somit können die rechtlichen Wertungen, die für das Reverse Engineering des Kommunikationsprotokolls ermittelt wurden, auch auf das Reverse Engineering der Payloads übertragen werden.

\section{Mitwirkungspflichten des Supportverpflichteten}

Die Wiederherstellung der Nutzungsmöglichkeit des Online-Mehrspielermodus ist zwar in rechtlicher Hinsicht entsprechend den bisher erläuterten Voraussetzungen weitgehend zulässig. Wie jedoch in Kapitel 1 erläutert wurde, ist das Reverse Engineering des Kommunikationsprotokolls und des Payloads ein sehr komplexes und zeitintensives Unterfangen. Darüber hinaus verfügt längst nicht jeder private Spieler, jeder Forscher oder jede Gedächtnisinstitution über das hierfür notwendige Fachwissen. Aus diesen Gründen kann die Wiederherstellung des Online-Mehrspielermodus schon aus tatsächlichen Gründen scheitern. Daher wird vor allem unter privaten Inhabern digitaler Spiele die Forderung laut, dass Supportverpflichtete die Informationen über die Spezifikationen des Kommunikationsprotokolls offenlegen. ${ }^{1092}$

\footnotetext{
1088 Burghardt-Richter/Bode, BB 2019, 2697, 2697.

1089 Vgl. die Begründung zum Regierungsentwurf, BT-Drs. 19/4724, S. 41.

1090 Burghardt-Richter/Bode, BB 2019, 2697, 2699.

1091 Siehe dazu oben, S. $28 \mathrm{ff}$.

1092 Siehe dazu oben, S. 30 f.
} 
Aus diesen Gründen ist zu untersuchen, ob Supportverpflichtete nach dem Ende des Supports der Online-Komponenten dazu verpflichtet sind, Informationen über die Spezifikationen des Kommunikationsprotokolls sowie der Paylods privaten Spielern, Forschern sowie Gedächtnisinstitutionen zwecks Wiederherstellung der Nutzungsmöglichkeit des Online-Mehrspielermodus offenzulegen bzw. zu lizenzieren.

Eine mit diesem Problem vergleichbare Diskussion betrifft die Frage, ob der Hersteller von Software dazu verpflichtet ist, dem Softwareerwerber den Quellcode der Software herauszugeben, damit dieser Änderungen, Fehlerberichtigungen oder Verbesserungen an der Software vornehmen kann. Es wird daher zuerst auf diese Diskussion eingegangen und untersucht, ob die Wertungen der Literatur und Rechtsprechung zu diesem Problem auf den hier behandelten Fall einer Offenlegungspflicht übertragen werden können (a)). Im Anschluss daran wird auf eine etwaige kartellrechtliche Pflicht des Supportverpflichteten zur Lizenzierung der Nutzung der Protokollinformationen eingegangen (b)).

\section{a) Vertragliche Pflicht zur Offenlegung der Protokollinformationen}

Software wird üblicherweise im maschinenlesbaren Objektcode vertrieben, welcher von Menschen nicht oder nur sehr schwer gelesen werden kann. ${ }^{1093}$ Die Erstellung der Software erfolgt demgegenüber im sog. Quellcode, mithilfe dessen Reparaturen oder Modifikationen am Programm möglich sind. ${ }^{1094}$ Zwar kann der maschinenlesbare Code theoretisch in den Quellcode wieder umgewandelt werden (sog. Dekompilieren), jedoch handelt es sich dabei um ein sehr schwieriges und zeitaufwändiges Verfahren, das noch dazu einen Quellcode hervorbringt, welcher mit dem ursprünglichen Quellcode nicht identisch ist und nur sehr eingeschränkt genutzt werden kann. ${ }^{1095}$

In Rechtsprechung und Literatur wird diskutiert, ob eine Pflicht des Softwareherstellers zur Herausgabe des Quellcodes an den Softwareerwerber besteht, damit der Erwerber Änderungen, Fehlerberichtigungen oder Verbesserungen an der Software vornehmen kann.

Rechtlich unproblematisch ist grundsätzlich die Situation, in welcher die Vertragsparteien sich darüber geeinigt haben, dass der Softwarehersteller zur Herausgabe des Quellcodes an den Programmanwender verpflichtet ist.

Problematisch sind dagegen die Szenarien, in welchen die Parteien eine solche Vereinbarung nicht getroffen haben. Hier ist zu überlegen, ob eine entsprechende Pflicht im Wege der ergänzenden Vertragsauslegung gemäß den $\S \S 133,157$ BGB hergeleitet werden kann.

\footnotetext{
1093 Vgl. Conrad, ITRB 2005, 12, 12.

1094 Ernst, MMR 2001, 208, 209; vgl. Wandtke/Bullinger/Grützmacher, § 69d UrhG, Rn. 21.

1095 Koch, Computer-Vertragsrecht, S. 492 f.
} 
Weitgehend Einigkeit besteht in Rechtsprechung und Literatur, dass eine Pflicht zur Herausgabe des Quellcodes nicht bei der Überlassung von Standardsoftware in Betracht kommt. ${ }^{1096}$ Hierunter werden im Allgemeinen Computerprogramme verstanden, die nicht für die individuellen Bedürfnisse der jeweiligen Abnehmer entwickelt oder angepasst werden. ${ }^{1097}$ Begründet wird dies damit, dass der Erwerber von Standardsoftware grundsätzlich nicht erwarte, dass er den Quellcode, der nicht ausschließlich für ihn geschaffen wurde, erhält und selbst in die Lage versetzt wird, Änderungen am Programm vorzunehmen. ${ }^{1098} \mathrm{Zu}$ diesem Zweck bestehe in der Regel vielmehr die Möglichkeit, gegen eine zusätzliche Vergütung einen Wartungsvertrag mit dem Hersteller des Programms abzuschließen. ${ }^{1099}$ Darüber hinaus müsse sich der Hersteller von Standardsoftware die Verwertungsmöglichkeiten des Quellcodes für die Zukunft sichern. ${ }^{100}$

Einer Minderansicht zufolge soll jedoch ein Anspruch auf Herausgabe des Quellcodes der Standardsoftware zumindest dann anzunehmen sein, wenn der Hersteller des Computerprogramms dessen Pflege nicht übernehmen will und die Pflege durch einen Dritten vorgenommen werden soll. ${ }^{1101}$

Demgegenüber wird in Literatur und Rechtsprechung mit Blick auf Individualsoftware eine vertragliche Pflicht - auch bei fehlender Vereinbarung - zur Überlassung des Quellcodes grundsätzlich angenommen. ${ }^{1102}$ Begründet wird diese Ansicht damit, dass der Auftragnehmer bei Individualsoftware nicht auf die Geheimhaltung des Quellcodes angewiesen sei, da die Auswertung der Software ohnehin nur einmal nämlich gegenüber dem Auftraggeber - erfolge. ${ }^{1103}$ Insbesondere dann, wenn kein Wartungsvertrag vereinbart wurde, habe der Auftraggeber ein großes Interesse daran, den Quellcode zu erhalten, um etwaige notwendige Änderungen, Wartungen und Fehlerberichtigungen vornehmen zu können. ${ }^{1104}$

1096 OLG München, CR 1992, 208, 209; Ernst, MMR 2001, 208, 209 f.; Koch, Computer-Vertragsrecht, S. 493; von dem Bussche/Schelinski, in Leupold/Glossner, Münchener Anwalts Handbuch IT-Recht, Teil 1, Rn. 283; Schneider, EDV-Recht, Teil M, Rn. 713.

1097 von dem Bussche/Schelinski, in Leupold/Glossner, Münchener Anwalts Handbuch IT-Recht, Teil 1, Rn. 56.

1098 OLG München, CR 1992, 208, 209; Ernst, MMR 2001, 208, 210.

1099 OLG München, CR 1992, 208, 209.

1100 Ernst, MMR 2001, 208, 209.

1101 Wieczorek, in Kilian/Heussen, Computerrechtshandbuch, Abschnitt 1, Teil 3, 32.5, Rn. 17; Redeker, IT-Recht, Rn. 315.

1102 BGH, NJW-RR 2004, 782, 782; OLG Karlsruhe, CR 1999, 11, 12; OLG Saarbrücken, BB 1995, 12, 13; LG Köln, CR 2000, 505, 505; LG Aschaffenburg, CR 1998, 203, 205; LG München, CR 1989, 990, 990; Redeker, IT-Recht, Rn. 315; von dem Bussche/Schelinski, in Leupold/Glossner, Münchener Anwalts Handbuch IT-Recht, Teil 1, Rn. 283; Koch, Computer-Vertragsrecht, S. 493 ff.; zurückhaltend Wieczorek, in Kilian/Heussen, Computerrechtshandbuch, Abschnitt 1, Teil 3, 32.5, Rn. 17.

1103 von dem Bussche/Schelinski, in Leupold/Glossner, Münchener Anwalts Handbuch IT-Recht, Teil 1, Rn. 283.

1104 Vgl. LG Aschaffenburg, CR 1998, 203, Rn. 206. 
Es gibt jedoch innerhalb dieser Meinungsgruppe unterschiedliche Ansichten darüber, unter welchen Voraussetzungen ein Herausgabeanspruch zu bejahen ist.

Nach einer Ansicht sei ein solcher Anspruch des Softwareerwerbers bereits dann anzunehmen, wenn der Hersteller der Software keine Wartungsverpflichtung übernommen hat und die Herausgabe des Quellcodes für die Anpassung, Wartung oder Fehlerbeseitigung erforderlich ist. ${ }^{1105}$

Einer weiteren Ansicht zufolge komme ein Herausgabeanspruch schon dann in Betracht, wenn der Auftraggeber dem Auftragnehmer umfassende Nutzungsrechte an der Individualsoftware einräumt. ${ }^{1106}$

Der BGH betont schließlich, dass eine Herausgabepflicht nur unter Berücksichtigung der Interessenlage beider Parteien bejaht werden könne. Für die Interessenabwägung relevante Umstände können beispielsweise die Preisgestaltung sein, ob der Auftraggeber beabsichtigt, die Software weiter zu vermarkten, oder ob die Herausgabe des Quellcodes für die Fehlerbeseitigungs-, Wartungs- und Änderungsarbeiten erforderlich ist. ${ }^{107}$

Bei digitalen Spielen entfällt eine Unterscheidung zwischen Standard- und Individualsoftware, da digitale Spiele für gewöhnlich nur in Masse produziert und vertrieben werden. Eine Anpassung des digitalen Spiels für einzelne Kunden erfolgt also nicht. Die Tatsache, dass digitale Spiele zwangsläufig als Standardsoftware im oben beschriebenen Sinne einzuordnen sind, rechtfertigt es allein jedoch nicht, einen Anspruch auf Offenlegung der Protokollinformationen von vornherein abzulehnen. Es kommt hier vielmehr auf eine Interessenabwägung an.

Für eine Offenlegungspflicht spricht zunächst, dass im Gegensatz zum Quellcode die Protokollinformationen keine umfassende Änderung oder Weiterentwicklung des digitalen Spiels, sondern eben nur eine Wiederherstellung des Online-Mehrspielermodus ermöglichen. Die Verwendungs- und Verwertungsmöglichkeiten der Protokollinformationen sind daher im Vergleich zum Quellcode wesentlich eingeschränkter.

Gegen eine Offenlegungspflicht spricht allerdings die Verkehrssitte: Es ist vollkommen unüblich, dass Supportverpflichtete digitaler Spiele Informationen über die eingesetzten Kommunikationsprotokolle an Spieler, Forscher oder Gedächtnisinstitutionen weitergeben bzw. lizenzieren, damit diese den Online-Mehrspielermodus wiederherstellen können. Jedenfalls ist ein solcher Fall nicht bekannt.

Eine derart uneingeschränkte Offenlegungspflicht würde darüber hinaus auch die Interessen des Publishers ungebührlich beeinträchtigen. Wenngleich mit dem Kommunikationsprotokoll keine umfassenden Änderungen oder Weiterentwicklungen des digitalen Spiels vorgenommen werden können, handelt es sich dabei dennoch um wichtiges Know-how, das dem Supportverpflichteten auf dem Markt einen Vorteil gegenüber seinen Mitbewerbern verschaffen kann. ${ }^{1108}$ Eine Offenlegungspflicht würde

\footnotetext{
1105 OLG Saarbrücken, BB 1995, 12, 13; LG Köln, CR 2000, 505, 505; LG Aschaffenburg, CR 1998, 203, 206.

1106 Seffer/Horter, ITRB 2005, 169, 171.

1107 BGH, NJW-RR 2004, 782, $782 \mathrm{f}$.

${ }^{1108}$ Siehe dazu oben, S. $188 \mathrm{ff}$.
} 
diesen Vorteil - auch nach dem Ende des Supports - beseitigen. Denn auch wenn der Supportverpflichtete für die Online-Komponenten eines konkreten Spiels keinen Support mehr erbringt, bedeutet dies nicht, dass das Kommunikationsprotokoll nicht auch für den Support des Online-Mehrspielermodus anderer, neuerer digitaler Spiele Verwendung findet. Würde man eine umfassende Offenlegungspflicht bejahen, würde das Know-how, welches im Kommunikationsprotokoll verkörpert ist, entwertet.

Die Abwägung des Interesses des Erwerbers des digitalen Spiels an der Offenlegung der Protokollinformationen mit dem Interesse des Supportverpflichteten an ihrer ungehinderten Verwertung für neue digitale Spiele spricht somit gegen eine im Wege der ergänzenden Vertragsauslegung zu ermittelnden Pflicht des Supportverpflichteten zur Offenlegung der Protokollinformationen.

\section{b) Kartellrechtliche Pflicht zur Lizenzierung der Protokollnutzung}

Das Kommunikationsprotokoll und seine Spezifikationen können als Betriebsgeheimnis ${ }^{1109}$ oder als Patent ${ }^{1110}$ Rechtsschutz genießen. In beiden Fällen kann der Schutzrechtsinhaber eine Erlaubnis zur Nutzung des Kommunikationsprotokolls im Rahmen eines Lizenzvertrages erteilen. ${ }^{1111}$

Der Rechtsinhaber mag im Einzelfall durchaus dazu bereit sein, die Nutzung des Kommunikationsprotokolls zum Zwecke der Wiederherstellung der Nutzungsmöglichkeit des Online-Mehrspielermodus durch Spieler, Forscher und Gedächtnisinstitutionen zu gestatten. Es ist allerdings auch sehr wahrscheinlich, dass er eine Lizenzierung ablehnt.

$\mathrm{Zu}$ beachten ist allerdings, dass das Recht des Schutzrechtsinhabers, darüber zu entscheiden, einen Lizenzvertrag abzuschließen, seine Grenze im Verbot des Missbrauchs einer marktbeherrschenden Stellung gemäß Art. 102 AEUV finden kann. ${ }^{112}$ Es stellt sich daher die Frage, ob die Weigerung des Rechtsinhabers, die Nutzung der Protokollinformationen nach dem Ende des Supports zum Zwecke der Wiederherstellung des Online-Mehrspielermodus zu gestatten, den Missbrauch einer marktbeherrschenden Stellung darstellt und der Rechtsinhaber deshalb zur Lizenzierung an die interessierten Akteure (private Inhaber der Spielkopien, Forscher und Gedächtnisinstitutionen) verpflichtet ist.

Sowohl der EuGH als auch das EuG haben sich in mehreren Entscheidungen bereits mit der Frage auseinandergesetzt, wann eine Lizenzverweigerung den Missbrauch einer marktbeherrschenden Stellung darstellt.

\footnotetext{
1109 Siehe dazu oben, S. $188 \mathrm{ff}$.

1110 Siehe dazu oben, S. $184 \mathrm{f}$.

1111 Vgl. zur Lizenzierung von Betriebs- und Geschäftsgeheimnissen Köhler/Bornkamm/Feddersen/Köhler, vor $\S \S 17-19$, Rn. 4 f.

1112 Ebd., Rn. 4a.
} 
Dabei gilt im Ausgangspunkt, dass die Weigerung des Abschlusses eines Lizenzvertrages als solche keine missbräuchliche Ausübung der immaterialgüterrechtlichen Befugnisse darstellt. ${ }^{113}$ Die Möglichkeit, Dritte an der Nutzung des geschützten Gegenstands ohne Erlaubnis des Rechtsinhabers zu hindern, gehört schließlich zur „Substanz" des ausschließlichen Rechts. ${ }^{1114}$ Der ständigen Rechtsprechung des EuGH zufolge müssen vielmehr ,,außergewöhnliche Umstände“ hinzutreten, um eine Lizenzverweigerung als einen Missbrauch einer marktbeherrschenden Stellung im Sinne des Art. 102 AEUV einordnen zu können. ${ }^{1115}$

\section{aa) Europäisches Kartellrecht}

In seiner Magill-Entscheidung hat der EuGH erläutert, welche Umstände dies sein können. Der Entscheidung lag ein Fall zugrunde, in welchem ein irisches Unternehmen - die Magill TV Guide Ltd. - einen Fernsehprogrammführer veröffentlichen wollte, welcher Informationen über die Fernsehprogramme verschiedener Fernsehanstalten bündelte. ${ }^{1116}$ Die Fernsehanstalten hatten jedoch bisher Programmführer veröffentlicht, die nur Informationen über ihr jeweils eigenes Programm enthielten. Die Fernsehanstalten lehnten es ab, Magill eine urheberrechtliche Lizenz einzuräumen, damit Letztere ihre Programmvorschauen in einem zusammengefassten Programmführer veröffentlichen konnte. Der EuGH erblickte in dieser Lizenzverweigerung einen Missbrauch gemäß Art. 86 Abs. 2 lit. b EG-Vertrag (heute Art. 102 Abs. 2 lit. b AEUV). ${ }^{1117}$ Die Lizenzverweigerung der Fernsehanstalten verhindere nämlich erstens die Einführung eines neuen Produktes, wofür eine potenzielle Nachfrage von Verbrauchern besteht, sei zweitens nicht gerechtfertigt und führe drittens dazu, dass die Rechtsinhaber sich einen abgeleiteten Markt vorbehielten, indem sie jeglichen Wettbewerb auf diesem Markt ausschließen. ${ }^{1118}$

In der später ergangenen IMS Health-Entscheidung stellte der EuGH klar, dass die genannten Kriterien kumulativ vorliegen müssen. ${ }^{119}$ Darüber hinaus sei es erforderlich, dass der Zugang zu dem durch ein Immaterialgüterrecht geschützten Gegenstand

\footnotetext{
1113 EuGH, GRUR Int. 1990, 141, 142, Tz. 8 - Volvo/Veng; GRUR Int. 1995, 490, 493, Tz. 49 Magill TV Guide; GRUR Int. 2004, 644, 646, Tz. 34 - IMS Health / NDC Health; von der Groeben/Schwarze/Hatje/Schröter/Bartl, Art. 102 AEUV, Rn. 312; Wilhelmi, WRP 2009, 1431, 1433.

1114 EuGH, GRUR Int. 1990, 141, 142, Tz. 8; vgl. auch BGH, NJW-RR 2005, 269, 272 - StandardSpundfass.

1115 EuGH, GRUR Int. 1995, 490, 493, Tz. 50; GRUR Int. 1990, 141, 142, Tz. 9; GRUR Int. 2004, 644, 646, Tz. 34.

1116 EuGH, GRUR Int. 1995, 490, 490, Tz. 9 ff.

1117 Ebd., 493, Tz. 54.

1118 Ebd., 493, Tz. 54 ff.

1119 EuGH, GRUR Int. 2004, 644, 646, Tz. 38.
} 
für die Tätigkeit auf dem abgeleiteten Markt unerlässlich ist, hierfür also ansonsten realistischerweise kein Ersatz vorhanden ist. ${ }^{1120}$

2004 stellte die EU-Kommission darüber hinaus in einer Entscheidung gegen das Unternehmen Microsoft unter Anwendung der Magill-Kriterien fest, dass Microsoft gegen Art. 82 EG-Vertrag verstieß, weil es sich geweigert hatte, seinen Konkurrenten Schnittstelleninformationen offenzulegen, um Server-Software herstellen und anbieten zu können, die mit dem Microsoft-Betriebssystem Windows interoperabel ist. ${ }^{1121}$ Das EuG bestätigte die Entscheidung der Kommission im Wesentlichen in seinem Urteil vom 17.09.2007.

\section{bb) Subsumtion}

Fraglich ist, ob unter Anwendung der soeben genannten Kriterien eine Pflicht für den Rechtsinhaber hergeleitet werden kann, nach dem Ende des Supports die Nutzung des Kommunikationsprotokolls zum Zwecke der Wiederherstellung des Online-Mehrspielermodus zu lizenzieren.

Voraussetzung ist zunächst, dass der Rechtsinhaber eine marktbeherrschende Stellung innehat. Hierbei gilt, dass die Inhaberschaft eines Immaterialgüterrechts allein noch nicht ausreicht, um eine marktbeherrschende Stellung zu begründen. ${ }^{1122}$ Die Feststellung einer Marktbeherrschung erfordert vielmehr die Abgrenzung des relevanten Marktes, die mithilfe des sog. Bedarfsmarktkonzeptes erfolgt. Hiernach gehören zu einem bestimmten Markt alle Produkte und Dienstleistungen, welche aus Sicht der Nachfrager hinsichtlich Eigenschaft, Verwendungszweck und Preislage zur Deckung eines bestimmten Bedarfs austauschbar sind. ${ }^{1123}$ Die Vergabe von Lizenzen in Bezug auf eine geschützte technische Lehre stellt dann einen abgegrenzten Markt dar, wenn die Herstellung eines Produktes mithilfe dieser technischen Lehre nicht durch andere technische Lehren substituiert werden kann und damit die Erlangung von Nutzungsrechten für die Herstellung dieses Produktes unerlässlich ist. ${ }^{1124}$

Eine Beherrschung dieses Marktes ist gegeben, wenn das jeweilige Unternehmen eine wirtschaftliche Machtstellung innehat,

,, die dieses in die Lage versetzt, die Aufrechterhaltung eines wirksamen Wettbewerbs auf dem relevanten Markt zu verhindern, indem sie ihm die Möglichkeit verschafft, sich seinen Wettbewerbern, seinen Abnehmern und letztlich den Verbrauchern gegenüber in einem nennenswerten Umfang unabhängig zu verhalten ". 125

\footnotetext{
1120 Wilhelmi, WRP 2009, 1431, 1434; vgl. EuGH, GRUR Int. 2004, 644, 647, Tz. 48 f.; LG Düsseldorf, Urt. v. 05.07.2007, Az. 4b O 289/06 - zit. nach juris, Tz. 83.

1121 Vgl. EuG, Urt. v. 17.09.2007, Az. T-201/04 - zit. nach juris, Tz. 36 - Microsoft.

1122 EuGH, GRUR Int. 1995, 490, 492, Tz. 46; Immenga/Mestmäcker/Fuchs/Möschel, Art. 102 AEUV, Rn. 365; Wilhelmi, WRP 2009, 1431, 1436.

1123 BGH, NJW-RR 2005, 269, 271.

1124 Vgl. ebd., 271f.; Beckmann/Müller, in Hoeren/Sieber/Holznagel, Handbuch Multimedia-Recht, Teil 10, Rn. 24.

1125 EuGH, BeckRS 2009, 70381, Rn. 103 - France Télécom.
} 
Unter Anwendung dieser Kriterien ist davon auszugehen, dass der Inhaber der Rechte am Kommunikationsprotokoll regelmäßig auf dem Markt der Vergabe von Lizenzen für die von ihm eingesetzten Kommunikationsprotokolle marktbeherrschend ist. Da für die Einrichtung und Aufrechterhaltung einer Client-Server-Kommunikation die exakte Übernahme der Protokollspezifikationen erforderlich ist, kann das eingesetzte - und durch Schutzrechte geschützte - Kommunikationsprotokoll nicht einfach durch ein anderes ersetzt werden. Die Einräumung einer Lizenz zur Nutzung des Kommunikationsprotokolls ist damit unerlässlich für die Wiederherstellung der Nutzungsmöglichkeit des Online-Mehrspielermodus.

Die Lizenzverweigerung muss weiterhin dazu führen, dass die Einführung eines neuen Produktes auf einem abgeleiteten Markt verhindert wird, wofür eine potenzielle Nachfrage von Verbrauchern besteht.

Dieses Kriterium dient dazu, für Verbraucher nachteilige Marktentwicklungen zu verhindern, die dadurch eintreten können, dass Wettbewerber des marktbeherrschenden Unternehmens infolge der Lizenzverweigerung keine neuen Erzeugnisse oder Dienstleistungen mehr anbieten können, wofür jedoch eine potenzielle Nachfrage der Verbraucher besteht. ${ }^{1126}$ Dogmatisch handelt es sich bei diesem Erfordernis um einen Ausgleich zwischen dem Interesse des Immaterialgüterrechtsinhabers an seiner wirtschaftlichen Handlungsfreiheit und dem Schutz des freien Wettbewerbs. ${ }^{1127}$ Im Falle einer Verhinderung der Entwicklung neuer Produkte überwiegt der Schutz des freien Wettbewerbs. Allerdings müssen durch die Lizenzierung neue Produkte oder Dienstleistungen entstehen. Würde vielmehr nur die Vermarktung von Imitationsprodukten angestrebt, würde durch eine Zwangslizenz unzulässigerweise in den Bestand des Immaterialgüterrechts eingegriffen. ${ }^{1128}$ In einem solchen Fall überwiegt grundsätzlich das Interesse des Immaterialgüterrechtsinhabers an seiner wirtschaftlichen Handlungsfreiheit. Das EuG hatte in seiner Microsoft-Entscheidung allerdings das Erfordernis der Verhinderung der Entwicklung eines neuen Produktes weit ausgelegt und auch die Einschränkung der technischen Entwicklung zum Schaden der Verbraucher - durch Ausbleiben der Entwicklung konkurrierender, kompatibler Betriebssystemprodukte für Arbeitsgruppenserver - genügen lassen. ${ }^{1129}$

Zwar gereicht auch die Weigerung der Lizenzierung der Nutzung der Kommunikationsprotokolle zum Nachteil der Verbraucher - insbesondere solcher, die bereits eine Spielkopie besitzen, deren Online-Mehrspielermodus nunmehr keinen Support erhält. Dennoch überwiegen hier die Interessen des Rechtsinhabers hinsichtlich seiner wirtschaftlichen Handlungsfreiheit. Die Lizenzierung der Nutzung des Kommunikationsprotokolls würde nämlich nicht zu einer wirtschaftlichen oder technischen Entwicklung von neuen Produkten oder Dienstleistungen führen, sondern lediglich die Lücke füllen, welche der Supportverpflichtete nach dem Ende des Supports der Online-Kom-

\footnotetext{
1126 EuGH, GRUR Int. 2004, 644, 647, Tz. 48 f.

1127 Ebd., 647, Tz. 48; Wilhelmi, WRP 2009, 1431, 1438 f.

1128 Vgl. de Bronett, in Wiedemann, Handbuch des Kartellrechts, § 22, Rn. 58.

${ }^{1129}$ EuG, Urt. v. 17.09.2007, Az. T-201/04 - zit. nach juris, Rn. 647.
} 
ponenten hinterlassen hat. Eine Zwangslizenz hätte also die Entstehung von Nachahmungsprodukten zur Folge. Müsste der Rechtsinhaber allerdings hinnehmen, dass sein Schutzrecht gegenüber einem imitierenden Wettbewerb zurücktritt, liefe sein Immaterialgüterrecht leer. ${ }^{1130}$

Hinzu kommt, dass es sehr häufig mehrere Auflagen von einem Spieletitel gibt. ${ }^{1131}$ Würde der Rechtsinhaber verpflichtet werden, die Nutzung des Kommunikationsprotokolls zu lizenzieren, könnte ihm der Anreiz genommen werden, den jeweiligen Spieletitel neu aufzulegen oder eine Fortsetzung hierfür zu produzieren.

Damit ergibt sich für den Rechtsinhaber aus dem europäischen Kartellrecht keine Pflicht zur Lizenzierung der Nutzung des Kommunikationsprotokolls zum Zwecke der Wiederherstellung des Online-Mehrspielermodus.

\section{cc) Deutsches Kartellrecht}

Gemäß Art. 3 Abs. 2 Satz 2 der VO 1/2003/EG darf das nationale Kartellrecht für einseitige Verhaltensweisen von Unternehmen strengere Regelungen als das europäische Kartellrecht vorsehen. In Betracht kommt dabei insbesondere die Missbrauchsaufsicht gemäß $\S 19$ GWB. ${ }^{1132}$

Zwar hat der deutsche Gesetzgeber gemäß § 19 Abs. 2 Nr. 4 GWB ein Regelbeispiel eingefügt, nach welchem ein Missbrauch einer marktbeherrschenden Stellung auch dann vorliegen kann, wenn Wettbewerbern die Tätigkeit auf bestimmten Märkten verwehrt ist, weil das marktbeherrschende Unternehmen ihnen den Zugang zu Netzen und anderen Infrastruktureinrichtungen verweigert. Aus diesem missbräuchlichen Verhalten kann schließlich auch ein Anspruch auf Zugangsgewährung der Wettbewerber erwachsen. ${ }^{1133}$

Allerdings hat der Gesetzgeber einen solchen Anspruch mit Blick auf die Nutzung gewerblicher Schutzrechte in der Gesetzesbegründung ausgeschlossen. ${ }^{1134}$ Dies gilt auch für die Generalklausel des Art. 19 Abs. 1 GWB; denn würde man hiernach bei einer missbräuchlichen Ausnutzung einer marktbeherrschenden Stellung eine Zwangslizenz bejahen, würde man die Wertungen des Gesetzgebers unterlaufen. ${ }^{1135}$

Insgesamt ist damit eine kartellrechtliche Lizenzierungspflicht des Inhabers der Rechte am Kommunikationsprotokoll nach dem Ende des Supports der Online-Komponenten sowohl nach europäischem als auch nach deutschem Kartellrecht abzulehnen.

\section{Der Betrieb des neuen Masterservers}

Nachdem die Informationen über das Kommunikationsprotokoll und die Payloads vom Reverse Engineerer ermittelt wurden, schließen sich als Nächstes die Einrichtung

\footnotetext{
${ }^{1130}$ Vgl. de Bronett, in Wiedemann, Handbuch des Kartellrechts, § 22, Rn. 58.

1131 Siehe dazu die Einleitung.

${ }^{1132}$ Immenga/Mestmäcker/Rehbinder, Art. 3 VO 1/2003, Rn. 32.

1133 Vgl. Immenga/Mestmäcker/Körber/Fuchs/Möschel, § 19 GWB, Rn. 345 ff.

1134 BT-Drs. 13/9720, S. 79 f.

1135 Gabriel/C. Cornels, ITRB 2008, 277, 278.
} 
und der Betrieb eines Masterservers an, mithilfe dessen der Support des Online-Mehrspielermodus wiederhergestellt werden kann.

Im Folgenden wird untersucht, ob der Betrieb des Masterservers sowie damit zusammenhängende Handlungen mit dem UWG (1.) und dem MarkenG (2.) vereinbar sind.

\section{Zulässigkeit nach dem UWG}

Initiativen privater Inhaber von Spielkopien, welche den Online-Mehrspielermodus wiederhergestellt haben, bieten grundsätzlich allen Inhabern einer Spielkopie an, eine Verbindung zwischen dem neu eingerichteten Masterserver und ihrer Spielkopie herzustellen. ${ }^{1136}$ Es ist darüber hinaus auch möglich, dass Gedächtnisinstitutionen sowie Forscher Masterserver einrichten, um den Online-Mehrspielermodus wiederherzustellen, wenngleich solche Fälle bislang noch nicht bekannt sind. Mit dem Betrieb eines „,inoffiziellen“ Masterservers, der den Online-Mehrspielermodus wiederherstellt, wird aber eine Dienstleistung angeboten, welche der Supportverpflichtete bis zur Beendigung des „offiziellen“ Supports in dieser oder ähnlicher Form auch selbst erbrachte und für andere digitale Spiele unter Umständen auch noch erbringt.

Diese Tatsache wirft die Frage auf, ob es sich bei dem Betrieb des Servers zum Zwecke der Wiederherstellung der Nutzungsmöglichkeit des Online-Mehrspielermodus um eine unlautere geschäftliche Handlung zulasten des Supportverpflichteten gemäß $§ 1$ S. 1 UWG handelt.

Voraussetzung hierfür ist das Vorliegen einer geschäftlichen Handlung im Sinne des $\S 2$ Abs. 1 Nr. 1 UWG. Sie muss bei objektiver Betrachtung darauf gerichtet sein, „durch Beeinflussung der geschäftlichen Entscheidung der Verbraucher oder sonstigen Marktteilnehmer den Absatz des Bezugs von Waren oder Dienstleistungen des eigenen oder eines fremden Unternehmens zu fördern"“. ${ }^{1137}$

An einer geschäftlichen Handlung i. S. d. § 2 Abs. 1 Nr. 1 UWG fehlt es bereits bei rein privaten Tätigkeiten, die nicht der Erwerbs- oder Berufsausübung einer Person zuzurechnen sind. ${ }^{1138}$ Aus diesem Grunde wird die Wiederherstellung des OnlineMehrspielermodus durch einen nicht genehmigten Masterserver, welcher von privaten Erwerbern eingerichtet und betrieben wird, nicht erfasst. Der Betrieb des Masterservers erfolgt hier allein zu dem Zweck, das jeweilige digitale Spiel innerhalb der Spielergemeinschaft wieder vollumfänglich nutzen zu können. Die Wiederherstellung steht damit in der Regel weder im Zusammenhang mit der Erwerbstätigkeit der Server-Betreiber noch mit der Förderung des Absatzes oder des Bezugs von Waren bzw. Dienstleistungen eines Unternehmens.

1136 Siehe dazu die Einleitung sowie S. 30 f.

1137 BGH, GRUR 2013, 945, 946, Tz. 17 - Standardisierte Mandatsbearbeitung; vgl. auch OLG Frankfurt a. M., GRUR-RR 2015, 298, 298, Tz. 8 - Community \& Forum; OLG Franfurt a. M., GRUR-RR 2016, 155, 155, Tz. 5 - WarnWetter-App.

${ }^{1138}$ Vgl. Köhler/Bornkamm/Feddersen/Köhler, § 2 UWG, Rn. 18; Ohly/Sosnitza/Sosnitza, § 2 UWG, Rn. 14; Harte-Bavendamm/Henning-Bodewig/Keller, § 2 UWG, Rn. 38. 
Fraglich ist aber, ob Gedächtnisinstitutionen, welche zur Wiederherstellung des Online-Mehrspielermodus den dafür erforderlichen Masterserver betreiben, eine geschäftliche Handlung im Sinne des $\S 2$ Abs. 1 Nr. 1 UWG vornehmen. Gedächtnisinstitutionen wie beispielsweise Bibliotheken in Deutschland stellen überwiegend Einrichtungen der öffentlichen Hand dar. ${ }^{1139}$ Ein hoheitliches Handeln, das aufgrund einer ausdrücklichen, gesetzlichen Ermächtigungsgrundlage oder Verpflichtung erfolgt, ist nicht als ein geschäftliches Handeln i. S. d. § 2 Abs. 1 Nr. 1 UWG einzuordnen. ${ }^{1140}$ Öffentlich-rechtliche Bibliotheken sind gesetzlich dazu verpflichtet, Medienwerke zu sammeln und ,auf Dauer zu sichern und für die Allgemeinheit nutzbar zu machen“.1141 Der Sammelauftrag von Gedächtnisinstitutionen umfasst mittlerweile auch digitale Objekte. ${ }^{1142}$ Bibliotheken sowie Museen in Deutschland sammeln zum Teil auch digitale Spiele ${ }^{1143}$. Neben dem Bestandsaufbau sind Bibliotheken auch zur Erhaltung und Vermittlung ihres Bestandes verpflichtet. ${ }^{144}$ Es ist allerdings keine gesetzliche Regelung ersichtlich, die Gedächtnisinstitutionen konkret und ausdrücklich auch zur Wiederherstellung des Online-Mehrspielermodus digitaler Spiele, die sich in ihrem Bestand befinden, verpflichten würde. Aus diesem Grunde kann die Einordnung des Betriebs eines Masterservers durch Bibliotheken zur Wiederherstellung des OnlineMehrspielermodus als geschäftliche Handlung nicht bereits deshalb verneint werden, weil es eine ausdrückliche gesetzliche Ermächtigungsgrundlage gibt, welche Bibliotheken zu dieser Maßnahme verpflichtet.

Zur Feststellung, ob der Betrieb eines Masterservers durch eine Gedächtnisinstitution eine geschäftliche Handlung i. S. d. § 2 Abs. 1 Nr. 1 UWG darstellt, kommt es daher darauf an, ob er objektiv mit der Förderung des Absatzes oder Bezugs von Waren oder Dienstleistungen zusammenhängt. ${ }^{1145}$ Es ist dabei grundsätzlich unerheblich,

1139 Moeske, Bibliotheksrecht (2009), abrufbar unter www.bibliotheksportal.de/themen/recht/bibliotheksrecht-allgemein.html.

1140 BGH, GRUR 2006, 428, 428 f., Tz. 12 - Abschleppkosten-Inkasso; Köhler/Bornkamm/Feddersen/Köhler, § 3a UWG, Rn. 2.21.

1141 Vgl. § 2 Nr. 1 des Gesetzes über die Deutsche Nationalbibliothek (DNBG); vgl. auch die Regelungen einzelner Bibliotheksgesetze in den Bundesländern, wie z. B. § 1 Abs. 2 des Gesetzes für die Bibliotheken in Schleswig-Holstein sowie $\S 3$ des Bibliotheksgesetzes des Landes Sachsen-Anhalt.

1142 So sammelt die Deutsche Nationalbibliothek beispielsweise auch unkörperliche Medienwerke, wie z.B. "ausgewählte Websites", Deutsche Nationalbibliothek, Sammlung unkörperlicher Medienwerke (FAQ) (2020), abrufbar unter https://www.dnb.de/DE/Professionell/Sammeln/Unkoerperliche_Medienwerke/unkoerperliche_ medienwerke_node.html\#doc210120bodyText2.

1143 So z. B. die Jugendbibliothek „Hallescher Komet“ der Zentral- und Landesbibliothek Berlin, https://www.zlb.de/kijubi/jugendbibliothek/ueber-uns/ueber-die-jubi.html oder das Computerspielemuseum in Berlin,

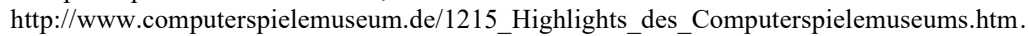

1144 Siehe z. B. § 2 Nr. 1 DNBG; ausführlich dazu Dreier/Euler/Fischer/van Raay, ZUM 2012, 273, $274 \mathrm{ff}$.

1145 Ohly/Sosnitza/Ohly, Einleitung D, Rn. 24. 
ob eine Gewinnerzielungsabsicht verfolgt ${ }^{1146}$ oder ob die Maßnahme gegen ein Entgelt erbracht wird ${ }^{1147}{ }^{1148}$ Je mehr die Handlung der Erfüllung öffentlicher Aufgaben dient und entsprechend die Absicht in den Hintergrund tritt, den Wettbewerb zu fördern, desto eher ist ein solcher Zusammenhang abzulehnen. ${ }^{1149}$

Ohne den Betrieb von Masterservern ist die Nutzungsmöglichkeit des Online-Mehrspielermodus nicht gegeben und die Nutzungsmöglichkeit der digitalen Spielkopie bestenfalls nur zu einem Teil vorhanden. Beim Betrieb der Masterserver handelt es sich also um eine für den Erhalt und die Vermittlung des Bestandes der Gedächtnisinstitution notwendige Maßnahme. Wie bereits dargestellt, fallen sowohl der Erhalt als auch die Vermittlung von Beständen in den Aufgabenbereich der Gedächtnisinstitutionen. Aus diesem Grund steht beim Betrieb von Masterservern durch Gedächtnisinstitutionen grundsätzlich die Erfüllung der öffentlichen Aufgabe des Erhalts und der Vermittlung von Beständen im Vordergrund und nicht die Förderung des Absatzes von Waren bzw. Dienstleistungen der Gedächtnisinstitution oder dritter Unternehmen. Daher stellt der Betrieb von Masterservern durch Gedächtnisinstitutionen zur Wiederherstellung der Nutzungsmöglichkeit des Online-Mehrspielermodus grundsätzlich keine geschäftliche Handlung i. S. d. § 2 Abs. 1 Nr. 1 UWG dar.

Auch der Betrieb von Masterservern zur Wiederherstellung des Online-Mehrspielermodus durch Forscher stellt in der Regel keine geschäftliche Handlung gemäß § 2 Abs. 1 Nr. 1 UWG dar. Es ist anzunehmen, dass der Betrieb des Servers und der Zugriff auf den Online-Mehrspielermodus hier ausschließlich innerhalb einer Forschungseinrichtung bzw. innerhalb eines Kreises von Forschern erfolgt, sodass es schon an einer für die geschäftliche Handlung erforderlichen Außenwirkung ${ }^{1150}$ fehlt. Selbst wenn aber eine solche Außenwirkung im Einzelfall bejaht werden sollte, fehlt es wiederum an einem objektiven Zusammenhang zwischen der Wiederherstellung des Online-Mehrspielermodus und der Förderung des Absatzes oder des Bezugs von Waren bzw. Dienstleistungen eines Unternehmens. Denn Handlungen, welche der Unterrichtung der Öffentlichkeit zu wissenschaftlichen Zielen dienen, fehlt es an diesem Zusammenhang. ${ }^{1151}$

\section{Zulässigkeit nach dem MarkenG}

Wie bereits in Kapitel 1 erläutert, erblicken Publisher in dem Betrieb privater Server und damit zusammenhängender Handlungen insbesondere eine Verletzung ihrer Markenrechte. ${ }^{1152}$

\footnotetext{
1146 Ebd., Rn. 25; BGH, GRUR 1982, 425, 430 - Brillen-Selbstabgabestellen.

${ }^{1147}$ LG Bonn, Urt. v. 15.11.2017 - Az. 16 O 21/16 - GRUR-RS 2017, 136665, Tz. 51 - WarnWetter-App; OLG Hamm, MMR 2012, 32, 33.

1148 BGH, WRP 1993, 106, 107 - EWG-Baumusterprüfung.

1149 Ohly/Sosnitza/Ohly, Einleitung D, Rn. 29.

1150 Siehe dazu Köhler/Bornkamm/Feddersen/Köhler, § 2 UWG, Rn. 35.

1151 Vgl. ebd., Rn. 51.

1152 Siehe dazu die Einleitung.
} 
Auch wenn die Publisher nicht erläutern, in welchen konkreten Fällen sie eine Verletzung ihrer Zeichenrechte annehmen würden, sind durchaus Szenarien denkbar, in denen eine nähere Prüfung einer etwaigen Verletzung von Zeichenrechten angezeigt ist.

Im Rahmen der Einrichtung und des Betriebs eines eigenen Masterservers ist es beispielsweise möglich, dass die Benutzeroberfläche des neuen Server-Browsers ${ }^{1153}$ mit dem geschützten Logo oder Titel des Spiels versehen wird.

Darüber hinaus ist es üblich, dass Projekte, die sich der Wiederherstellung des Online-Mehrspielermodus digitaler Spiele widmen, Webseiten lancieren, die Informationen über das Projekt und darüber, wie die einzelnen Spieler ihre funktionslose Spielkopie wieder funktionstüchtig machen können, enthalten. In diesem Zusammenhang erfolgt zwangsläufig ein Hinweis auf den Spieletitel ${ }^{1154}$, unter Umständen auch eine Einbettung von Logos des betroffenen digitalen Spiels in Überschriften, Bannern und anderen grafischen Gestaltungselementen der Webseiten.

Schließlich ist es auch möglich, dass sich der Spieletitel als Bestandteil im DomainNamen wiederfindet. In all diesen Fällen würden die Spieler bzw. die an der Wiederherstellung des Supports beteiligten Personen etwaige geschützte Marken und Werktitel der Publisher verwenden.

Fraglich ist, ob in solchen Handlungen auch eine Verletzung der Markenrechte von Publishern zu erblicken ist.

Voraussetzung ist hierfür zunächst eine Benutzung der Marke bzw. des Werktitels des digitalen Spiels im geschäftlichen Verkehr. ${ }^{155}$ Dafür ist erforderlich, dass „die Benutzung im Zusammenhang mit einer auf einen wirtschaftlichen Vorteil gerichteten kommerziellen Tätigkeit und nicht im privaten Bereich" ${ }^{\text {"156 }}$ erfolgt. An die Benutzung im geschäftlichen Verkehr sind keine strengen Anforderungen zu stellen, sodass es auch nicht darauf ankommt, ob eine Gewinnerzielungsabsicht vorliegt. ${ }^{157}$

Die Einrichtung und der Betrieb der Masterserver erfolgen in der Regel durch die Inhaber der Spielkopien im rein privaten Bereich, ohne dass sie mit diesen Tätigkeiten kommerzielle Zwecke verfolgen. Im Einzelfall kann jedoch eine Benutzung der Marke bzw. des Werktitels im geschäftlichen Verkehr anzunehmen sein, wenn das geschützte Zeichen auf einer privat betriebenen Webseite platziert wird und dort zusätzlich Banner-Werbung erscheint. ${ }^{158}$

\footnotetext{
1153 Näheres zum Begriff oben in der Einleitung.

1154 So z. B. auf SaveMGO, Frequently Asked Questions (2020), abrufbar unter https://savemgo.com/forums/app.php/help/faq.

${ }^{1155}$ Die Kriterien einer Benutzung des Werktitels im geschäftlichen Verkehr gleichen den Krtierien für dieses Tatbestandsmerkmal in Bezug auf Marken, vgl. BeckOK Markenrecht/Mielke, § 15 MarkenG, Rn. 15 f.

1156 EuGH, GRUR 2010, 445, 447, Tz. 50 - Google France.

1157 BeckOK Markenrecht/Mielke, § 14 MarkenG, Rn. 55.

1158 Vgl. ebd., Rn. 75.
} 
Grundsätzlich nicht im geschäftlichen Verkehr erfolgen allerdings Handlungen zu rein wissenschaftlichen Zwecken. ${ }^{159}$ Aus diesem Grunde stellen Einrichtung und Betrieb eines ,inoffiziellen“ Masterservers zur Wiederherstellung des Online-Mehrspielermodus zu Forschungszwecken keine Benutzung der Marken im geschäftlichen Verkehr dar. Eine Benutzung der Marken durch öffentlich-rechtliche Gedächtnisinstitutionen im Rahmen des Betriebs der Masterserver zur Erhaltung und Vermittlung von Beständen digitaler Spiele stellt grundsätzlich ebenfalls keine Benutzung der Marke im geschäftlichen Verkehr dar, weil hoheitliches Handeln grundsätzlich nicht von diesem Begriff erfasst wird. ${ }^{1160}$

Sofern eine Benutzung der Marke im geschäftlichen Verkehr im Einzelfall aber zu bejahen ist, verlangt eine weitere Voraussetzung für etwaige Ansprüche des Markeninhabers gemäß $\S 14$ Abs. 5 bis 7 MarkenG, dass der in Anspruch genommene Verletzer die geschützten Kennzeichen auch markenmäßig verwendet hat. Hierfür ist Voraussetzung, dass die benutzten Kennzeichen zur Unterscheidung von Waren oder Dienstleistungen eines Unternehmens von denen anderer Unternehmen dienen. ${ }^{161}$ Der Schutz der Marke ist nämlich auf die Fälle beschränkt, in denen ihre Funktionen insbesondere die Herkunftsfunktion - durch eine unzulässige Kennzeichenbenutzung Dritter beeinträchtigt werden (könnten). ${ }^{1162}$

In den oben beschriebenen Fällen werden die als Marken geschützten Spieletitel verwendet, um die Herkunft der digitalen Spiele zu bestimmen, auf die sich die Wiederherstellung des Online-Mehrspielermodus bezieht. Damit hat die Benutzung der Marken den Zweck, das digitale Spiel, dessen Online-Mehrspielermodus wieder rekonstruiert werden soll, von anderen digitalen Spielen zu unterscheiden. Da die Marken somit dazu genutzt werden, um die Herkunft der digitalen Spiele zu unterscheiden, liegt auch eine markenmäßige Benutzung vor.

Für Ansprüche des Markeninhabers gegen einen Verletzer der Marke ist weiterhin Voraussetzung, dass einer der Tatbestände des $\S 14$ Abs. 2 Nr. 1-3 MarkenG erfüllt ist.

In den hier zu betrachtenden Fällen kommt regelmäßig eine sog. Doppelidentität der verwendeten Zeichen sowie der Waren bzw. Dienstleistungen gemäß § 14 Abs. 2 Nr. 1 MarkenG in Betracht. So liegt es nahe, dass Projekt-Webseiten, welche auf die Wiederherstellung des Online-Mehrspielermodus bestimmter Spiele hinweisen, auch die Marken dieser Spieletitel in unveränderter Form - beispielsweise in Hinweistexten oder im Rahmen der generellen Aufmachung der Webseite (z. B. in Überschriften oder sogar als Teil des Domainnamens) - übernehmen. Darüber hinaus werden Dienstleistungen angeboten, die mit denen identisch sind, für welche die Marken der Publisher bzw. Entwickler Schutz genießen.

\footnotetext{
1159 BGH, GRUR 2004, 241, 242 - GeDIOS.

1160 Spindler/Schuster/Müller, § 14 MarkenG, Rn. 60.

1161 BGH, GRUR 2008, 793, 794, Tz. 15 - Rillenkoffer; EuGH, GRUR Int. 1999, 438, 441, Tz. 38 $B M W$.

1162 BGH, GRUR 2008, 793, 794, Tz. 15.
} 
Aus den gleichen Gründen ist auch eine Verwirklichung des Tatbestandes des $\S 15$ Abs. 2 MarkenG mit Blick auf Werktitel anzunehmen. Die Benutzung eines identischen Unternehmenskennzeichens wird von diesem Tatbestand, der ausdrücklich nur die Verwechslungsgefahr wegen Ähnlichkeit der Zeichen regelt, mit umfasst. ${ }^{1163}$

Auch wenn die Verletzungstatbestände des $\S 14$ Abs. 2 Nr. 1 und $\S 15$ Abs. 2 MarkenG erfüllt sind, hat dies aber noch nicht zwangsläufig zur Folge, dass der Inhaber der Marke bzw. des Werktitels die Nutzung untersagen darf. Zu beachten ist nämlich, dass $\S 23$ MarkenG bestimmte Nutzungen von Marken und Unternehmenskennzeichen für zulässig erklärt.

In Betracht kommt hier $\S 23$ Abs. 1 Nr. 3 MarkenG. Hiernach dürfen eine Marke bzw. ein Werktitel ,zu Zwecken der Identifizierung oder zum Verweis auf Waren oder Dienstleistungen als die des Inhabers der Marke [benutzt werden], insbesondere wenn die Benutzung der Marke als Hinweis auf die Bestimmung einer Ware insbesondere als Zubehör oder Ersatzteil oder einer Dienstleistung erforderlich ist“".

Die Benutzung einer Marke zu dem Zweck, die Öffentlichkeit darauf hinzuweisen, dass sich beispielsweise Wartungs- und Instandsetzungsdienstleistungen auf bestimmte Markenwaren oder -Dienstleistungen beziehen, ist damit grundsätzlich erlaubt. ${ }^{164}$ Die Benutzung der Marke muss allerdings für den Bestimmungshinweis notwendig sein. Dies bedeutet, dass die Benutzung der Marke praktisch das einzige Mittel dafür darstellt, um der Öffentlichkeit eine verständliche und vollständige Information über die Bestimmung der vom Dritten vertriebenen Waren oder erbrachten Dienstleistungen zu liefern. ${ }^{1165}$

Die Benutzung darf außerdem nicht gegen die "anständigen Gepflogenheiten in Gewerbe und Handel" verstoßen, vgl. § 23 Abs. 2 MarkenG. Dieses Tatbestandsmerkmal verlangt, dass berechtigte Interessen des Markeninhabers der Benutzung der Marke bzw. des Werktitels nicht entgegenstehen dürfen. ${ }^{1166}$ Dies ist insbesondere dann der Fall, wenn entweder

(1) durch die Benutzung der Eindruck entsteht, dass eine Handelsbeziehung zwischen dem Dritten und dem Markeninhaber existiert, (2) durch die Benutzung der Wert der Marke bzw. des Werktitels dadurch beeinträchtigt wird, dass sie deren Unterscheidungskraft oder deren Wertschätzung in unlauterer Weise ausnutzt, (3) durch die Benutzung eine Herabsetzung der Marke bzw. des Werktitels erfolgt, oder (4) der Dritte seine Ware als eine Nachahmung der Markenware darstellt. ${ }^{1167}$

Unter Berücksichtigung dieser Kriterien sind solche Benutzungen von der Schranke des $\S 23$ Abs. 1 Nr. 3 MarkenG erfasst sein, bei denen die Informationsvermittlung im Vordergrund steht und welche die Benutzung der Marke bzw. des Werktitels auf das

\footnotetext{
1163 Vgl. BeckOK Markenrecht/Thalmaier, § 15 MarkenG, Rn. 31.

1164 EuGH, GRUR Int. 1999, 438, 442, Tz. 59.

1165 EuGH, GRUR 2005, 509, 511 f., Tz. 34 ff. - Gillette; Lange, Markenrecht, Rn. 3651.

1166 EuGH, GRUR 2005, 509, 512 f., Tz. 49; BeckOK Markenrecht/Kretschmar, § 23 MarkenG, Rn. 46; Lange, Markenrecht, Rn. 3654.

1167 EuGH, GRUR 2005, 509, 513, Tz. 49.
} 
zu diesem Zweck notwendige Maß beschränken. Dies sind beispielsweise Informations- und Hinweistexte auf den Projektwebseiten, welche die als Wortmarken geschützten Spieletitel verwenden, um darauf hinzuweisen, welcher Online-Mehrspielermodus von welchem Spiel wiederhergestellt worden ist.

Nicht mehr unter die Schranke fällt dagegen die Verwendung von Wortbildmarken, da es für den Hinweis nach $\S 23$ Abs. 1 Nr. 3 MarkenG in der Regel ausreichen dürfte, allein den Spieletitel als solchen und damit die Wortmarke zu verwenden. ${ }^{1168}$

Unzulässig und damit nicht von der Schranke gedeckt ist auch die Verwendung der Marke als Bestandteil des Domain-Namens, da der Nutzer hier in unzulässiger Weise vom Ansehen der Marke profitiert und sich dieses zunutze macht, ohne dass ein Verweis auf die Markenprodukte im Rahmen des Domain-Namens erforderlich ist. ${ }^{1169}$

Die soeben durchgeführte zeichenrechtliche Prüfung steht jedoch unter dem Vorbehalt, dass nach wie vor noch ein zeichenrechtlicher Schutz für den Spieletitel besteht.

Mit Blick auf Werktitel ist dabei zu beachten, dass der Schutz desselben von seiner Benutzung durch den Berechtigten abhängt. Mit der endgültigen Aufgabe der Benutzung des Werktitels durch seinen Berechtigten entfällt grundsätzlich auch das Recht an diesem. ${ }^{1170}$ Dies gilt jedoch erst ab dem Zeitpunkt, zu dem das digitale Spiel endgültig nicht mehr neu auf dem Markt erworben werden kann. ${ }^{171}$

Darüber hinaus kann gemäß $\S 25$ Abs. 1 MarkenG der Inhaber einer eingetragenen Marke keine Ansprüche gemäß $\S 14$ MarkenG geltend machen, wenn die Marke innerhalb der letzten fünf Jahre vor der Geltendmachung des Anspruchs nicht gem. § 26 MarkenG benutzt worden ist, sofern zu diesem Zeitpunkt seit mindestens fünf Jahren kein Widerspruch mehr gegen die Marke möglich war.

Der Anspruchsgegner des Markeninhabers muss den Einwand der Nichtbenutzung als Einrede im Prozess geltend machen. ${ }^{1172}$ Der Benutzungszwang verlangt, dass die Marke ,auf dem Markt der durch sie geschützten Waren oder Dienstleistungen benutzt wird““ ${ }^{1173}$ Die Benutzung der Marke muss sich dabei auf Waren beziehen, die „,bereits vertrieben werden“ oder deren Vertrieb „unmittelbar bevorsteht““ ${ }^{1174}$ Dies ist bei digitalen Spielen, deren Support von Online-Komponenten eingestellt wurde, jedoch in der Regel nicht der Fall. Denn mit der Beendigung des Supports der Online-Komponenten geht in der Regel auch eine Einstellung des Vertriebs der entsprechenden digitalen Spiele einher. In solchen Fällen kann daher eine mangelnde Benutzung im Sinne des $\S 25$ Abs. 1 MarkenG in Betracht kommen. Anders kann die Rechtslage allerdings dann zu bewerten sein, wenn die Marke einen Serientitel schützt, der aus mehreren

\footnotetext{
1168 Vgl. dazu BGH, GRUR 2011, 1135, 1138, Tz. 26 - GROSSE INSPEKTION FÜR ALLE.

1169 Vgl. Lange, Markenrecht, Rn. 3656.

1170 BeckOK Markenrecht/Weiler, § 5 MarkenG, Rn. 250.

1171 Vgl. ebd., Rn. 247; vgl. auch Körner, in Duisberg/Picot, Recht der Computer- und Videospiele, Kap. 3, Rn. 60.

1172 BeckOK Markenrecht/Bogatz, § 25 MarkenG, Rn. 27.

1173 EuGH, GRUR 2003, 425, 427 f., Tz. 37 - Ansul/Ajax.

1174 Ebd., 427f., Tz. 37.
} 
Nachfolge-Spieletiteln besteht, und neuere Spiele unter diesem Serientitel noch vertrieben werden.

Insgesamt kann daher festgehalten werden, dass die Benutzung der Marken bzw. Werktitel des Publishers im Rahmen der Wiederherstellung des Online-Mehrspielermodus - beispielsweise in Hinweis- und Informationstexten auf Projektwebseiten - in der Regel bereits keine markenmäßige Benutzung darstellt, weil es an einer Benutzung der Marke für Waren und Dienstleistungen im geschäftlichen Verkehr fehlt.

Doch selbst im Falle der Bejahung einer markenmäßigen Benutzung im geschäftlichen Verkehr dürfte in der Regel die Schranke des $\S 23$ Abs. 1 Nr. 3 MarkenG eingreifen, welche die Benutzung von Marken und Werktiteln zum Zwecke des Hinweises auf die Bestimmung der angebotenen Dienstleistung zulässt. Dabei muss sich die Benutzung der Marke aber im Rahmen des Erforderlichen bewegen und darf diesen nicht überschreiten. Zu beachten ist darüber hinaus, dass im Einzelfall auch die Einrede der Nichtbenutzung der Marke durch den Publisher in Betracht kommen kann.

\section{Zulässigkeit der Wiederherstellung der Nutzungsmöglichkeit sup- portloser digitaler Spiele nach US-amerikanischem Copyright law}

Mit der Frage, ob die Wiederherstellung der Nutzungsmöglichkeit supportloser digitaler Spiele zulässig ist, hatte sich das Copyright Office in den USA im Rahmen des sog. Sixth Triennial Section 1201 Proceeding befasst.

Dieses - im Folgenden auch „Rechtsetzungsverfahren“ genannte - Verfahren wurde bereits in der Einleitung kurz dargestellt und soll im Folgenden sowohl hinsichtlich seiner Hintergründe als auch seiner Ergebnisse näher erläutert werden.

Wie auch das deutsche und europäische Urheberrecht verbietet der US-amerikanische Copyright $\mathrm{Act}^{1175}$ die Umgehung technischer Maßnahmen zum Schutze von urheberrechtlich geschützten Werken. Sec. 1201 des Copyright Act wurde durch den Digital Millenium Copyright Act (DMCA) 1998 eingeführt. ${ }^{1176}$

Sec. 1201(a)(1)(C) des Copyright Act überträgt dem Librarian of Congress die Aufgabe, im Rahmen eines Rechtsetzungsverfahrens Gruppen von urheberrechtlich geschützten Werken im Sinne des sec. 1201(a)(1)(B) zu bestimmen, die vom Verbot der Umgehung technischer Schutzmaßnahmen ausgenommen werden sollen. Gemäß sec. 1201(a)(1)(C) sollen solche Werke vom Umgehungsverbot ausgenommen werden, deren Nutzer ansonsten in ihrem rechtmäßigen Gebrauch dieser Werke eingeschränkt werden würden. Ein solch rechtmäßiger Gebrauch kann sich z. B. aus der Schrankennorm des sec. 107 ergeben, welcher die fair use-Klausel beinhaltet. ${ }^{1177}$

\footnotetext{
117517 U.S.C. sec. 1201(a)(1)(A).

$1176 \mathrm{Vgl}$. Bechtold, Informationsrecht, S. $207 \mathrm{f}$.

1177 Vgl. US Copyright Office, Understanding the Section 1201 Rulemaking (2015), 2, abrufbar unter http://copyright.gov/1201/2015/2015_1201_FAQ_final.pdf.
} 
Die Bestimmung solcher Werkkategorien erfolgt alle drei Jahre auf Empfehlung vom Direktor des Copyright Office, dem Register of Copyrights - zur Zeit des hier behandelten Rechtsetzungsverfahrens war dies Maria A. Pallante.

Das Rechtsetzungsverfahren beginnt mit einem öffentlichen Aufruf an die Bevölkerung der USA, Vorschläge für urheberrechtlich geschützte Werke einzureichen, die vom Verbot des sec. 1201(a)(1)(A) ausgenommen werden sollen. Alle sich daran anschließenden Verfahrensschritte können auf der Webseite des Copyright Office verfolgt werden und umfassen u. a. die Einsendung und Auswertung schriftlicher Stellungnahmen und öffentliche Anhörungen. Den Abschluss bilden die Empfehlung der Register of Copyrights und die Final Rule des Librarian of Congress. Dabei ist zu beachten, dass die beschlossenen Ausnahmen lediglich bis zum nächsten Rechtsetzungsverfahren gelten und sodann - sofern sich für die Erneuerung der Ausnahme keine Fürsprecher melden, welche die Ausnahmen erneut erfolgreich durchsetzen außer Kraft treten. ${ }^{1178}$

2015 fand das sechste Rechtsetzungsverfahren seit dem Inkrafttreten des DMCA im Jahre 1998 statt. In diesem Verfahren hatte die $E F F$ zusammen mit der Harvard Law School-Studentin Kendra Albert vorgeschlagen, die Umgehung von technischen Maßnahmen für digitale Spiele zu erlauben, sofern der Zweck dieser Umgehung darin besteht, den Einzel- oder den Mehrspielermodus des Spiels wieder nutzbar zu machen, nachdem der Entwickler den Support für diese Online-Komponenten eingestellt hat. ${ }^{1179}$ Im Verlaufe des Rechtsetzungsverfahrens unterstützten nicht nur Forscher und Gedächtnisinstitutionen die Petition, sondern es meldeten sich auch mehr als 1230 Einzelpersonen ${ }^{1180}$ schriftlich zu Wort, von denen ein Großteil private Inhaber von Kopien digitaler Spiele war. Die Ansichten und Standpunkte der Interessengruppen mit Blick auf die Wiederherstellung der Nutzungsmöglichkeit supportloser digitaler Spiele, die im Rahmen des Rechtsetzungsverfahrens vorgebracht wurden, sind bereits in Kapitel 1 dieser Arbeit ausführlich dargestellt worden.

Der Librarian of Congress hatte schließlich am 20. Oktober 2015 mit seiner Final Rule das Rechtsetzungsverfahren abgeschlossen.

Auf Grundlage der Empfehlung Pallantes entschied der Librarian of Congress, dass solche Kopien digitaler Spiele vom Umgehungsverbot ausgenommen werden, deren Nutzungsmöglichkeit infolge der Einstellung des Betriebs von Authentication-Servern wegfällt. ${ }^{1181}$ Sowohl Privatpersonen als auch Gedächtnisinstitutionen dürfen bei solchen Spielkopien nunmehr Vervielfältigungen und Veränderungen vornehmen, die

\footnotetext{
1178 Vgl. ebd., 3.

1179 Vgl. Electronic Frontier Foundation, Petition of Electronic Frontier Foundation and Kendra Albert (2014), 1, abrufbar unter http://copyright.gov/1201/2014/petitions/Electronic_Frontier_Foundation_and_Kendra_Albert_ 1201_Initial_Submission_2014.pdf.

1180 Register of Copyrights, Recommendation of the Register of Copyrights (2015), S. 322, abrufbar unter http://copyright.gov/1201/2015/registers-recommendation.pdf.

1181 Librarian of Congress, Federal Register, Vol. 80, No. 208 (2015), S. 65957, abrufbar unter http://copyright.gov/fedreg/2015/80fr65944.pdf.
} 
notwendig sind, um die Nutzungsmöglichkeit des Spiels wiederherzustellen. ${ }^{1182}$ Der Zweck kann dabei darin bestehen, entweder das Spiel privat wieder spielen zu können oder Erhaltungsmaßnahmen von Gedächtnisinstitutionen zu ermöglichen, solange dies zu nicht kommerziellen Zwecken geschieht und das Spiel nicht außerhalb der Räumlichkeiten der Gedächtnisinstitution vertrieben wird. ${ }^{1183}$ Unter den gleichen Bedingungen dürfen ausschließlich Gedächtnisinstitutionen ein eventuell erforderliches Jailbreaking von Spielekonsolen vornehmen, um die Nutzungsmöglichkeit von Konsolenspielen wiederherzustellen. ${ }^{184}$

Die EFF und Albert begründeten ihre Petition maßgeblich damit, dass es sich bei den für die Wiedernutzbarmachung supportloser digitaler Spiele notwendigen Maßnahmen um „faire Nutzungen“ im Sinne der fair use-Klausel gemäß sec. 107 des Copyright Act handle. ${ }^{1185}$ Zur Bestimmung dessen, ob eine Nutzung als fair und damit nicht als Rechtsverletzung anzusehen ist, erfolgt die Prüfung im Einzelfall anhand von vier Faktoren:

1. der Zweck und die Art der Nutzung, einschließlich der Frage, ob die Nutzung zu kommerziellen oder zu nicht kommerziellen Bildungszwecken erfolgt (the purpose and character of the use, including whether such use is of commercial nature or is for nonprofit educational purposes),

2. die Natur des geschützten Werkes (the nature of the coyrighted work),

3. der Umfang und die Bedeutung des genutzten Teils des Werks im Verhältnis zum geschützten Werk als Ganzes (the amount and substantiality of the portion used in relation to the copyrighted work as a whole) und

4. die Auswirkung der Nutzung des geschützten Werks auf seine potenzielle Verwertung oder seinen Wert (the effect of the use upon the potential market for or value of the copyrighted work).

Die $E F F$ und Albert sahen den ersten Faktor als erfüllt an, weil die Wiedernutzbarmachung dazu diene, die Interoperabilität zwischen rechtmäßig erworbenen Kopien digitaler Spiele und neuen Servern herzustellen und weil diese Wiedernutzbarmachung zu nicht kommerziellen Zwecken erfolge. ${ }^{1186}$ Die Herstellung von Interoperabilität sei dabei ein Zweck, der vom (amerikanischen) Copyright law erwünscht sei.

\footnotetext{
1182 Ebd.

1183 Ebd., S. 65957.

1184 Ebd.

1185 Electronic Frontier Foundation, Comments of the Electronic Frontier Foundation (2015), S. 6ff., abrufbar unter http://copyright.gov/1201/2015/comments020615/InitialComments_longform_EFF_Class23.pdf.

1186 Ebd., S. 7.
} 
Darüber hinaus sei auch der zweite Faktor erfüllt, da die Wiedernutzbarmachung der Spielkopie letztlich nur die Änderung funktionaler Aspekte der Spiele software erfordere, welche nur in geringem Maße Urheberrechtsschutz genießen. ${ }^{1187} \mathrm{Da}$ es sich auBerdem bei den zum Zwecke der Wiedernutzbarmachung zu ändernden Programmbestandteilen nur um kleine Teile des Computerprogramms handle, sei auch der dritte Faktor erfüllt. ${ }^{1188}$

Der vierte Faktor spreche ebenfalls für eine Einordnung der Wiedernutzbarmachung von supportlosen digitalen Spielen, da dadurch der Wert der digitalen Spiele steige und eine Beeinträchtigung der Verwertung ohnehin nicht mehr in Betracht kommen könne, wenn das Werk vom Rechtsinhaber nicht mehr vertrieben wird. ${ }^{1189}$

Insgesamt hätte die erstrebte Ausnahme aus Sicht von EFF und Albert positive Effekte für Wissenschaft und Forschung, aber auch für Spielergemeinschaften, die oftmals die Supportzeiträume der Publisher überdauerten. ${ }^{1190}$

Pallante schloss sich der Einschätzung der Antragenden hinsichtlich der Eliminierung nicht spielbezogener Online-Komponenten im Grundsatz an. Allerdings spreche der vierte Faktor nicht für den Jailbreak von Spielekonsolen. ${ }^{1191}$ Mithilfe eines Jailbreaks könnten Spielekonsolen letztlich auch dafür verwendet werden, Raubkopien digitaler Spiele zu nutzen, deren Support noch nicht beendet worden ist. ${ }^{1192}$ Dadurch würde der Wert der Spielekonsole als Distributionsplattform insgesamt geschmälert. ${ }^{1193} \mathrm{Im}$ Gegensatz dazu könne jedoch der Jailbreak von Spielekonsolen durch Gedächtnisinstitutionen zur Wiedernutzbarmachung supportloser digitaler Spiele als eine faire Nutzung angesehen werden, solange sich diese Nutzung auf die Räumlichkeiten dieser Einrichtungen beschränkt, die noch dazu diese Handlungen ausschließlich zu nicht kommerziellen Zwecken vornehmen. ${ }^{1194}$

Pallante beschied demgegenüber die Petition für eine Erlaubnis der Umgehung technischer Maßnahmen zum Zwecke der Wiederherstellung von spielbezogenen OnlineKomponenten negativ. ${ }^{1195}$ Die wesentlichen Gründe hierfür waren zum einen, dass die Nutzungsmöglichkeit hier nicht aufgrund einer serverbasierten technischen Maßnahme wegfällt, sondern wegen eines externen, vom Publisher betriebenen Services, der nunmehr eingestellt wurde. ${ }^{196}$ Die Petition von EFF und Albert scheint insofern nicht in den Anwendungsbereich des § 1201(a)(1)(A) des Copyright Act zu fallen.

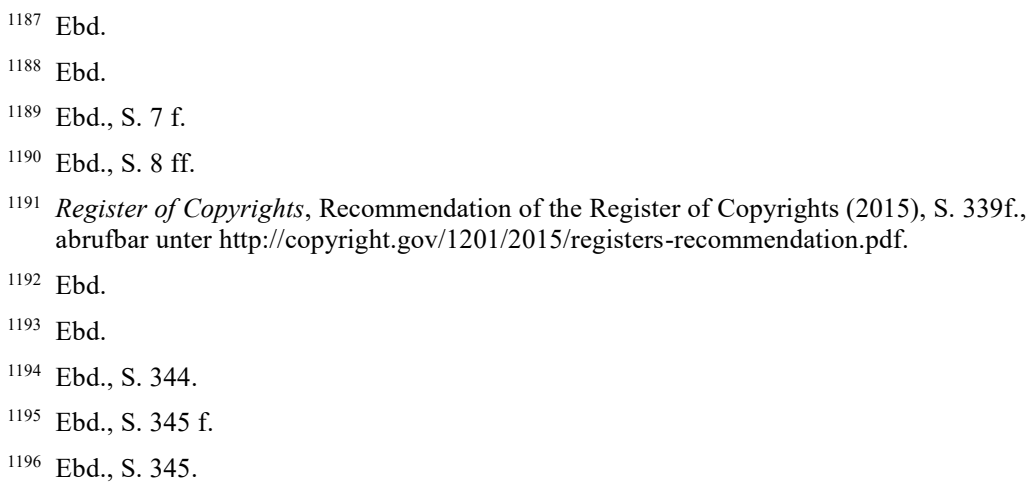


Darüber hinaus war Pallante der Auffassung, dass letztlich in vielen Fällen der Online-Mehrspielermodus durch einen LAN-Mehrspielermodus ${ }^{1197}$ in gleichwertiger Weise ersetzt werden könne. ${ }^{1198}$ Schließlich deutete Pallante an, dass die Nachschöpfung und Implementierung der Kommunikationsprotokolle sowie der Betrieb unautorisierter Server gegen die Vorschrift des sec. 1201(a)(2) verstoße, welche es insbesondere untersagt, Produkte oder Dienstleistungen anzubieten, die primär dazu bestimmt sind, technische Maßnahmen zu umgehen. ${ }^{1199}$

Im Jahr 2018 hat das Copyright Office das Seventh Triennial Section 1201 Proceeding eingeleitet. ${ }^{1200}$ Alex Handy, der Gründer und Direktor des The Museum of Art and Digital Entertainment, hat im Rahmen dieses Verfahrens beantragt, dass die 2015 erlassene Ausnahme supportloser digitaler Spiele vom Umgehungsverbot erneuert wird und damit für weitere drei Jahre in Kraft bleibt. ${ }^{1201}$

\section{E. Zusammenfassung}

Die Zulässigkeit der Wiederherstellung der Nutzungsmöglichkeit supportloser digitaler Spiele hängt davon ab, ob Online-DRM-Systeme, die als technische Maßnahmen im Sinne des $\S 95 \mathrm{a}$ Abs. 2 UrhG bzw. als Programmschutzmechanismen gemäß $\S 69 f$ Abs. 2 UrhG eingeordnet werden, betroffen sind oder vielmehr der Online-Mehrspielermodus.

Die Online-DRM-Systeme dürfen zum Zwecke der Wiederherstellung der Nutzungsmöglichkeit der Spielkopie nicht eliminiert werden. Zwar kann die Entfernung supportloser Programmschutzmechanismen als Fehlerberichtigung gemäß $§ 69 \mathrm{~d}$ Abs. 1 UrhG angesehen werden, jedoch setzt sich $\S 95$ a Abs. 1 UrhG, welcher die Umgehung technischer Maßnahmen verbietet, gegenüber dieser Vorschrift durch. Dies gilt unabhängig davon, welche Personengruppe die Eliminierung vornimmt: Spieler, Forscher oder Gedächtnisinstitutionen. Darüber hinaus ist es auch unzulässig, Anleitungen oder Umgehungssoftware zu verbreiten bzw. anzubieten, die eine Hilfestellung zur Umgehung der supportlosen Online-DRM-Systeme bieten sollen.

Grundsätzlich anders ist die Rechtslage dagegen hinsichtlich der Wiederherstellung des Online-Mehrspielermodus zu beurteilen. Mit Blick auf Spieler sind sowohl die Verbindung der Spielkopie mit einem neuen Server, das Reverse Engineering von

\footnotetext{
${ }^{1197}$ Siehe zum Begriff oben, S. 19.

1198 Register of Copyrights, Recommendation of the Register of Copyrights (2015), S. 345, abrufbar unter http://copyright.gov/1201/2015/registers-recommendation.pdf.

1199 Ebd., S. 346.

1200 Copyright Office, Seventh Triennial Section 1201 Proceeding (2018), abrufbar unter https://www.copyright.gov/1201/2018/.

${ }^{1201}$ Handy, Comment of the Museum of Art and Digital Entertainment (2018), S. 2ff., abrufbar unter https://www.copyright.gov/1201/2018/comments-121817/class8/class-08initialcomments-made.pdf.
} 
Kommunikationsprotokoll und Payload als auch der Serverbetrieb und weitere Maßnahmen wie informatorische Hinweise im Netz auf den neuen Server zulässig, sofern die Maßnahmen zu privaten und nicht kommerziellen Zwecken vorgenommen werden. Die Implementierung von patentrechtlich geschützten Kommunikationsprotokollen durch Forscher und Gedächtnisinstitutionen, welche die Spezifikationen des Protokolls im Wege des Reverse Engineering ermittelt hatten, stellt jedoch eine Patentverletzung dar.

Im Falle des Fehlens einer vertraglichen Vereinbarung über die Offenlegung des Kommunikationsprotokolls und der Lizenzierung der Nutzung desselben scheidet die Herleitung einer solchen Pflicht sowohl nach den Grundsätzen der ergänzenden Vertragsauslegung als auch unter kartellrechtlichen Gesichtspunkten aus. Eine solche Pflicht würde den Imitations-, nicht jedoch den Substitutionswettbewerb fördern und die Schutzrechte des Rechtsinhabers am Kommunikationsprotokoll leerlaufen lassen.

Während somit nach deutschem und europäischem Recht die Wiederherstellung des Supports der Online-Komponenten weitgehend zulässig, die Eliminierung der supportlosen serverbasierten Online-DRM-Systeme dagegen nicht erlaubt ist, stellt sich die Situation in den Vereinigten Staaten - zumindest aus urheberrechtlicher Sicht - genau umgekehrt dar. Im Rahmen des oben erläuterten Rechtsetzungsverfahrens plädierte die Register of Copyrights, Pallante, zwar für eine Ausnahme vom Verbot der Umgehung technischer Maßnahmen mit Blick auf supportlose serverbasierte Online-DRM-Systeme. Pallante sprach sich jedoch gegen eine Erlaubnis für eine Wiederherstellung der Nutzungsmöglichkeit des Online-Mehrspielermodus aus - wohl schon deshalb, weil mangels technischer Maßnahmen, die eine Nutzungsmöglichkeit einschränken würden, der Librarian of Congress eine solche Erlaubnis nach sec. 1201(a)(1)(C) gar nicht erteilen könnte. Nach Ansicht der Register of Copyrights bestehen darüber hinaus oftmals auch gleichwertige alternative Möglichkeiten, wie z. B. der LAN-Mehrspielermodus, welche den Online-Mehrspielermodus ersetzen könnten.

\section{Fazit}

Die Beendigung des Supports des Online-Mehrspielermodus oder von OnlineDRM-Systemen kann zu nicht unerheblichen Einschränkungen der Nutzungsmöglichkeit des digitalen Spiels führen. Die Tatsache, dass Supportverpflichtete den Support dieser Online-Komponenten recht willkürlich und zu nicht vorhersehbaren Zeiten beenden, verstärkt eine bei privaten Inhabern von Spielkopien, Forschern und Gedächtnisinstitutionen vorhandene Unsicherheit darüber, ob überhaupt ein Anspruch auf Support besteht und wie lange der Support der Online-Komponenten erbracht werden muss.

Die Arbeit hat gezeigt, dass dieser Rechtsunsicherheit keinesfalls eine Rechtslosigkeit der Erwerber digitaler Spiele gegenübersteht. Selbst wenn es an einer ausdrücklichen Regelung im Überlassungsvertrag fehlt, kann eine Pflicht zum Support im Wege der ergänzenden Vertragsauslegung ermittelt werden, die typologisch grundsätzlich dem Dienstvertragsrecht untergeordnet werden kann. 
Es gilt jedoch zu beachten, dass eine pauschale Aussage darüber, wann welche Supportpflichten entstehen, angesichts des sehr heterogenen Marktes digitaler Spiele nicht möglich ist. Es kommt daher vielmehr auf den Einzelfall und - insbesondere - auf die Art des digitalen Spiels an. Handelt es sich also beispielsweise um ein digitales Spiel, bei dem alle Online-Komponenten bereits nach dem Erwerb Support erhalten sollen oder vielmehr um ein Spiel, bei dem für den Support des Online-Mehrspielermodus etwa der Abschluss eines zusätzlichen, entgeltpflichtigen Vertrages erforderlich ist? Nach dieser Unterscheidung bestimmt sich letztlich, wann für wen eine Pflicht zum Support der Online-Komponenten entsteht.

Im deutschen Recht kann sich dabei - wiederum abhängig von der Art des digitalen Spiels - ein Ausschluss der Ausübung des ordentlichen Kündigungsrechts des Supportverpflichteten als Ergebnis einer Abwägung der Interessen des Erwerbers des digitalen Spiels und des Supportverpflichteten ergeben. Es besteht nämlich ein enger funktioneller Zusammenhang zwischen dem Spielclient und dem Support der OnlineKomponenten. Dieser enge funktionelle Zusammenhang, die Erwartung des Erwerbers, das Spiel für einen längeren Zeitraum nutzen zu können und die faktische Unfähigkeit des Erwerbers, mit dem Supportverpflichteten einen Kündigungsausschluss zu vereinbaren, rechtfertigen bei bestimmten digitalen Spielen bzw. virtuellen Gegenständen einen temporären Ausschluss der Ausübung des ordentlichen Kündigungsrechts des Supportverpflichteten in Bezug auf den Support der Online-Komponenten.

Die Wiederherstellung des Online-Mehrspielermodus ist grundsätzlich dann zulässig, wenn sie nicht zu kommerziellen Zwecken und nur im privaten Bereich vorgenommen wird. Ganz anders stellt sich dagegen die Situation bei supportlosen OnlineDRM-Systemen dar. Hier wird das für digitale Spiele bereits seit Längerem bekannte Problem der rechtlichen Behandlung urheberrechtlich geschützter, komplexer, multimedialer Werke besonders deutlich: Während die Eliminierung der supportlosen Online-DRM-Systeme - welche letztlich alle Bestandteile des digitalen Spiels schützen - gemäß $\S 69$ d Abs. 1 UrhG grundsätzlich zulässig ist, handelt es sich bei der Beseitigung solcher Online-DRM-Systeme zugleich um eine unzulässige Umgehung technischer Maßnahmen i. S. d. § 95a Abs. 1 UrhG. Da sowohl die Regelungen der $\S \S 69 \mathrm{a}$ ff. UrhG als auch der $\S \S 95$ a ff. UrhG auf digitale Spiele - und damit auch auf die technischen Maßnahmen - Anwendung finden, setzt sich das strengere Regelungsregime durch mit der Folge, dass die Eliminierung der supportlosen Online-DRM-Systeme insgesamt unzulässig ist.

Ein Anspruch nach § 95b Abs. 2 UrhG gegen den Rechtsinhaber auf Zurverfügungstellung von Mitteln zur Umgehung technischer Maßnahmen steht außerdem lediglich Forschern und Gedächtnisinstitutionen unter bestimmten Umständen zu, nicht jedoch privaten Nutzern digitaler Spiele. Ein Anspruch auf Lizenzierung der Nutzung des Kommunikationsprotokolls besteht - sofern er nicht ausdrücklich vertraglich geregelt ist - ebenfalls nicht. 
Open Access Dieses Kapitel wird unter der Creative Commons Namensnennung 4.0 International Lizenz (http://creativecommons.org/licenses/by/4.0/deed.de) veröffentlicht, welche die Nutzung, Vervielfältigung, Bearbeitung, Verbreitung und Wiedergabe in jeglichem Medium und Format erlaubt, sofern Sie den/die ursprünglichen Autor(en) und die Quelle ordnungsgemäß nennen, einen Link zur Creative Commons Lizenz beifügen und angeben, ob Änderungen vorgenommen wurden. Die in diesem Kapitel enthaltenen Bilder und sonstiges Drittmaterial unterliegen ebenfalls der genannten Creative Commons Lizenz, sofern sich aus der Abbildungslegende nichts anderes ergibt. Sofern das betreffende Material nicht unter der genannten Creative Commons Lizenz steht und die betreffende Handlung nicht nach gesetzlichen Vorschriften erlaubt ist, ist für die oben aufgeführten Weiterverwendungen des Materials die Einwilligung des jeweiligen Rechteinhabers einzuholen. 